\title{
Asymptotics of solutions of second order parabolic equations near conical points and edges
}

\author{
Vladimir A Kozlov ${ }^{1}$ and Jürgen Rossmann ${ }^{2 *}$
}

\section{"Correspondence:}

juergen.rossmann@uni-rostock.de ${ }^{2}$ Institute of Mathematics, University of Rostock, Rostock, 18051,

Germany

Full list of author information is

available at the end of the article

\begin{abstract}
The authors consider the first boundary value problem for a second order parabolic equation with variable coefficients in a domain with conical points or edges. In the first part of the paper, they study the Green function for this problem in the domain $K \times \mathbb{R}^{n-m}$, where $K$ is an infinite cone in $\mathbb{R}^{m}, 2 \leq m \leq n$. They obtain the asymptotics of the Green function near the vertex $(n=m)$ and edge $(n>m)$, respectively. This result is applied in the second part of the paper, which deals with the initial-boundary value problem in this domain. Here, the right-hand side $f$ of the differential equation belongs to a weighted $L_{p}$ space. At the end of the paper, the initial-boundary value problem in a bounded domain with conical points or edges is studied.
\end{abstract}

\section{Introduction}

The present paper is concerned with an initial-boundary value problem for a second order parabolic equation in a $n$-dimensional domain with a $(n-m)$-dimensional edge $M, n>$ $m \geq 2$. In particular, we are interested in the asymptotics of the solution near the edge. The largest part of the paper deals with the problem

$$
\begin{aligned}
& \partial_{t} u(x, t)-L\left(x, t, \partial_{x}\right) u(x, t)=f(x, t) \quad \text { for } x \in \mathcal{D}, t>0, \\
& \left.u\right|_{\partial \mathcal{D} \times \mathbb{R}_{+}}=0,\left.\quad u\right|_{t=0}=0
\end{aligned}
$$

in the domain $\mathcal{D}=K \times \mathbb{R}^{n-m}$. Here

$$
K=\left\{x^{\prime} \in \mathbb{R}^{m}: \omega=x^{\prime} /\left|x^{\prime}\right| \in \Omega\right\},
$$

is an infinite cone (angle if $m=2$ ), $\Omega$ is a subdomain of the unit sphere $S^{m-1}$ with $C^{1,1}$ boundary $\partial \Omega$, and

$$
L\left(x, t, \partial_{x}\right)=\sum_{i, j=1}^{n} a_{i j}(x, t) \partial_{x_{i}} \partial_{x_{j}}+\sum_{j=1}^{n} a_{j}(x, t) \partial_{x_{j}}+a_{0}(x, t),
$$

is a linear second order differential operator with variable coefficients.

Initial-boundary value problems for parabolic equations in domains with angular or conical points and edges were studied in a number of papers. Most of these papers deal

(C) 2014 Kozlov and Rossmann; licensee Springer. This is an Open Access article distributed under the terms of the Creative Commons Attribution License (http://creativecommons.org/licenses/by/4.0), which permits unrestricted use, distribution, and reproduction in any medium, provided the original work is properly credited. 
with the heat equation. Concerning the heat equation in domains with angular or conical points, we mention the papers by Grisvard [1], Kozlov and Maz'ya [2], de Coster and Nicaise [3], where the asymptotics of the solutions near the singular boundary points was studied. For domains with edges, Solonnikov $[4,5]$ and Nazarov [6] estimated the Green function and proved the existence of solutions of the Dirichlet and Neumann problems for the heat equation in weighted Sobolev spaces. Kozlov and Rossmann [7, 8] and Kweon [9] investigated the asymptotics of solutions of the Dirichlet problem for the heat equation near an edge.

A theory for general parabolic problems with time-independent coefficients in domains with conical points was developed in papers by Kozlov [10-12]. This theory includes the asymptotics of solutions in weighed $L_{2}$ Sobolev spaces and a description of the singularities of the Green function near the conical points. The goal of the present paper is to extend these results to the case of time-dependent coefficients and to domains with edges. Moreover, we consider solutions in weighted $L_{p}$ Sobolev spaces with arbitrary $p \in(1, \infty)$. However, we restrict ourselves to second order parabolic equations, and we consider only the first terms in the asymptotics. In our previous paper [13], we obtained point estimates for the Green function. These estimates together with results of the theory of elliptic boundary value problems are used in the present paper in order to describe the behavior of solutions near the edge.

We outline the main results of the present paper. For an arbitrary point $x=\left(x_{1}, \ldots, x_{n}\right) \in$ $\mathbb{R}^{n}$, we put $x^{\prime}=\left(x_{1}, \ldots, x_{m}\right)$ and $x^{\prime \prime}=\left(x_{m+1}, \ldots, x_{n}\right)$. The same notation is used for multiindices $\alpha=\left(\alpha_{1}, \ldots, \alpha_{n}\right)$. We assume that $a_{i j}=a_{j i}$ are real-valued functions and that

$$
\left|a_{i j}(x, t)-a_{i j}(0,0)\right| \leq \epsilon, \quad\left|a_{i}(x, t)\right| \leq \epsilon\left|x^{\prime}\right|^{-1}, \quad\left|a_{0}(x, t)\right| \leq \epsilon\left|x^{\prime}\right|^{-2},
$$

where $\epsilon$ is a small positive number. Besides this assumption, we impose some smoothness conditions on the coefficients $a_{i j}$ and $a_{j}$ (see (21) and (22)). The condition (3) ensures in particular that the difference of the operators $L\left(x, t, \partial_{x}\right)$ and

$$
L_{0}\left(0,0, \partial_{x}\right)=\sum_{i, j=1}^{n} a_{i j}(0,0) \partial_{x_{i}} \partial_{x_{j}}
$$

is small in the operator norm $W_{p ; \beta}^{2,1}\left(\mathcal{D}_{T}\right) \rightarrow L_{p ; \beta}\left(\mathcal{D}_{T}\right)$. Here $W_{p ; \beta}^{2 l, l}\left(\mathcal{D}_{T}\right)$ is the Sobolev space on $\mathcal{D}_{T}=\mathcal{D} \times(0, T)$ with the norm

$$
\|u\|_{W_{p ; \beta}^{2 l, l}\left(\mathcal{D}_{T}\right)}=\left(\int_{0}^{T} \int_{\mathcal{D}_{2 k+|\alpha| \leq 2 l}}\left|x^{\prime}\right|^{p(\beta-2 l+2 k+|\alpha|)}\left|\partial_{t}^{k} \partial_{x}^{\alpha} u(x, t)\right|^{p} d x d t\right)^{1 / p} .
$$

For $l=0$, this space is denoted by $L_{p ; \beta}\left(\mathcal{D}_{T}\right)$.

In Sections 2 and 3, we deal with the asymptotics of the Green function near the edge $M$ of $\mathcal{D}$. In the case of constant coefficients $a_{i j}$, the asymptotics can easily be obtained by means of the asymptotics of the Green function for the heat equation which is given in [7, 8]. If the coefficients are variable, then the terms in the asymptotics contain the eigenvalues and eigenfunctions of the following operator pencil $\mathfrak{A}\left(x^{\prime \prime}, t ; \lambda\right)$ :

$$
\mathfrak{A}\left(x^{\prime \prime}, t ; \lambda\right) \Phi(\omega)=\left|x^{\prime}\right|^{2-\lambda} L_{0}\left(0, x^{\prime \prime}, t, \partial_{x^{\prime}}, 0\right)\left|x^{\prime}\right|^{\lambda} \Phi(\omega), \quad \Phi \in \stackrel{\circ}{W_{2}^{1}}(\Omega) .
$$


Let $\lambda_{1}^{+}\left(x^{\prime \prime}, t\right)$ be the smallest positive eigenvalue and let $\Phi_{1}^{+}\left(x^{\prime \prime}, t ; \omega\right)$ be the corresponding eigenfunction. As was proved in [13], the Green function $G(x, y, t, \tau)$ of the problem (1) satisfies the estimate

$$
|G(x, y, t, \tau)| \leq c(t-\tau)^{-n / 2}\left(\frac{\left|x^{\prime}\right|}{\left|x^{\prime}\right|+\sqrt{t-\tau}}\right)^{\lambda}\left(\frac{\left|y^{\prime}\right|}{\left|y^{\prime}\right|+\sqrt{t-\tau}}\right)^{\lambda} \exp \left(-\frac{\kappa|x-y|^{2}}{t-\tau}\right)
$$

for $0<t-\tau<T,\left|\alpha^{\prime}\right| \leq 2,\left|\gamma^{\prime}\right| \leq 2$, where $\lambda<\lambda_{1}^{+}(0,0)-C \sqrt{\epsilon}$. Analogous estimates are valid for the derivatives of $G$ (cf. Theorem 3.1). Using this result, we show in Section 3 (see Theorem 3.2) that $G(x, y, t, \tau)$ admits the decomposition

$$
G(x, y, t, \tau)=\psi_{1}\left(x^{\prime \prime}, y, t, \tau\right)\left|x^{\prime}\right|^{\lambda_{1}^{+}\left(x^{\prime \prime}, t\right)} \Phi_{1}^{+}\left(x^{\prime \prime}, t ; \omega\right)+R(x, y, t, \tau)
$$

where

$$
|R(x, y, t, \tau)| \leq c(t-\tau)^{-n / 2}\left(\frac{\left|x^{\prime}\right|}{\sqrt{t-\tau}}\right)^{\mu}\left(\frac{\left|y^{\prime}\right|}{\sqrt{t-\tau}}\right)^{\lambda} \exp \left(-\frac{\kappa\left(\left|y^{\prime}\right|^{2}+\left|x^{\prime \prime}-y^{\prime \prime}\right|^{2}\right)}{t-\tau}\right)
$$

for $0<t-\tau<T$ and $\left|x^{\prime}\right|<\sqrt{t-\tau}$. Here, $\mu$ is a certain number greater than $\sup \lambda_{1}^{+}\left(x^{\prime \prime}, t\right)$. The coefficient $\psi_{1}$ in (6) satisfies the estimate

$$
\left|\psi_{1}\left(x^{\prime \prime}, y, t, \tau\right)\right| \leq c(t-\tau)^{-\left(n+\lambda_{1}^{+}\left(x^{\prime \prime}, t\right)\right) / 2}\left(\frac{\left|y^{\prime}\right|}{\sqrt{t-\tau}}\right)^{\lambda} \exp \left(-\frac{\kappa\left(\left|y^{\prime}\right|^{2}+\left|x^{\prime \prime}-y^{\prime \prime}\right|^{2}\right)}{t-\tau}\right)
$$

for $0<t-\tau<T$. Moreover, $\psi_{1}$ admits the decomposition

$$
\psi_{1}\left(x^{\prime \prime}, y, t, \tau\right)=\Psi_{1,0}\left(x^{\prime \prime}, y, t, \tau\right)+r_{1}\left(x^{\prime \prime}, y, t, \tau\right),
$$

where $\Psi_{1,0}$ is the function (37) and

$$
\left|r_{1}\left(x^{\prime \prime}, y, t, \tau\right)\right| \leq c(t-\tau)^{-\left(n-1+\lambda_{1}^{+}\left(x^{\prime \prime}, t\right)\right) / 2}\left(\frac{\left|y^{\prime}\right|}{\sqrt{t-\tau}}\right)^{\lambda} \exp \left(-\frac{\kappa\left(\left|y^{\prime}\right|^{2}+\left|x^{\prime \prime}-y^{\prime \prime}\right|^{2}\right)}{t-\tau}\right)
$$

for $0<t-\tau<T$.

In Section 4, we apply the results of the foregoing section in order to describe the behavior of the solutions of the problem (1) near the edge $M$. By Theorem 4.2, the following result holds. Suppose that $f \in L_{p ; \beta}\left(\mathcal{D}_{T}\right)$, where

$$
\sup \lambda_{1}^{+}\left(x^{\prime \prime}, t\right)<2-\beta-m / p<\lambda_{1}^{+}(0,0)+1-C \sqrt{\epsilon}, \quad 2-\beta-m / p<\inf \lambda_{2}^{+}\left(x^{\prime \prime}, t\right)-\sqrt{\epsilon}
$$

Then the solution of the problem (1) admits the decomposition

$$
u(x, t)=\left(\mathcal{E} h_{1}\right)(x, t)\left|x^{\prime}\right|^{\lambda_{1}^{+}\left(x^{\prime \prime}, t\right)} \Phi_{1}^{+}\left(x^{\prime \prime}, t ; \omega\right)+v(x, t),
$$

where

$$
h_{1}\left(x^{\prime \prime}, t\right)=\int_{0}^{t} \int_{\mathcal{D}} \psi_{1}\left(x^{\prime \prime}, y, t, \tau\right) f(y, \tau) d y d \tau
$$


$\mathcal{E}$ is the extension operator introduced in Section 4.2, and $v \in W_{p ; \beta}^{2,1}\left(\mathcal{D}_{T}\right)$. Note that the function $h_{1}$ belongs to the anisotropic Sobolev-Slobodetskii space $W_{p}^{s, s / 2}\left(\mathbb{R}^{n-m} \times(0, T)\right)$, where $s$ is the function $s\left(x^{\prime \prime}, t\right)=2-\beta-\lambda_{1}^{+}\left(x^{\prime \prime}, t\right)-m / p$.

Section 5 in closing deals with the initial-boundary value problem in a bounded domain with an edge. Under some smoothness conditions on the coefficients of the differential operator, we obtain the same decomposition of the weak solution near an edge point as in the case of the previously considered domain $\mathcal{D}$ (see Theorem 5.1 at the end of the paper).

\section{Green function of parabolic equations with constant coefficients}

In this section, we assume that

$$
L_{0}\left(\partial_{x}\right)=\sum_{i, j=1}^{n} a_{i, j} \partial_{x_{i}} \partial_{x_{j}}=\nabla_{x}^{T} A \nabla_{x}
$$

where $a_{i j}$ are real numbers, $a_{i j}=a_{j i}$ for all $i, j$. Here $A$ denotes the square matrix with the elements $a_{i, j}, \nabla_{x}$ is the nabla operator and $\nabla_{x}^{T}$ its transposed, i.e., $\nabla_{x}^{T}$ is the row vector with the components $\partial_{x_{j}}$.

There exists a coordinate transformation $\xi=S x$ with a constant square matrix $S$ such that the problem

$$
\partial_{t} u(x, t)-L_{0}\left(\partial_{x}\right) u(x, t)=f(x, t) \quad \text { for } x \in \mathcal{D}, t \in \mathbb{R},\left.\quad u\right|_{\partial \mathcal{D} \times \mathbb{R}_{+}}=0,
$$

takes the form

$$
\partial_{t} u-\Delta_{\xi} u=f \quad \text { for } \xi \in \mathcal{D}^{\prime}=K^{\prime} \in \mathbb{R}^{n-m}, t \in \mathbb{R},\left.\quad u\right|_{\partial \mathcal{D}^{\prime} \times \mathbb{R}}=0
$$

in the new coordinates $\xi$, where $K^{\prime}$ is a certain cone in $\mathbb{R}^{m}$ with vertex at the origin. This coordinate transformation can be constructed as follows. Let $A^{\prime}$ be the matrix with the elements $a_{i j}, i, j=1, \ldots, m, A^{\prime \prime}$ the matrix with the elements $a_{i j}, i, j=m+1, \ldots, n$, and $B$ the matrix with the elements $a_{i j}, i=1, \ldots, m, j=m+1, \ldots, n$. Furthermore, let $\nabla_{x^{\prime}}$ and $\nabla_{x^{\prime \prime}}$ be the nabla operators in the coordinates $x^{\prime}$ and $x^{\prime \prime}$, respectively. Then the operator $L_{0}$ can be written as

$$
L_{0}\left(\partial_{x}\right)=\nabla_{x^{\prime}}^{T}\left(A^{\prime} \nabla_{x^{\prime}}+B \nabla_{x^{\prime \prime}}\right)+\nabla_{x^{\prime \prime}}^{T}\left(B^{T} \nabla_{x^{\prime}}+A^{\prime \prime} \nabla_{x^{\prime \prime}}\right) .
$$

There exists an invertible matrix $U$ such that $U A^{\prime} U^{T}=I_{m}$ (the $m \times m$ identity matrix). This is true for the matrix $U=\Lambda^{-1 / 2} V$, where $\Lambda$ is the diagonal matrix of the (positive) eigenvalues of the matrix $A^{\prime}$ and the rows of $V$ are the orthonormalized eigenvectors of $A^{\prime}$. For the coordinates $y^{\prime}=U x^{\prime}, y^{\prime \prime}=x^{\prime \prime}-B^{T} A^{\prime-1} x^{\prime}$, we have $\nabla_{x^{\prime}}=U^{T} \nabla_{y^{\prime}}-A^{\prime-1} B \nabla_{y^{\prime \prime}}, \nabla_{x^{\prime \prime}}=\nabla_{y^{\prime \prime}}$ and, consequently,

$$
L_{0}\left(\partial_{x}\right)=\Delta_{y^{\prime}}+\nabla_{y^{\prime \prime}}^{T}\left(A^{\prime \prime}-B^{T} A^{\prime-1} B\right) \nabla_{y^{\prime \prime}}
$$

Obviously, the transformation $\left(x^{\prime}, x^{\prime \prime}\right) \rightarrow\left(U x^{\prime}, x^{\prime \prime}-B^{T} A^{\prime-1} x^{\prime}\right)=\left(y^{\prime}, y^{\prime \prime}\right)$ maps $K \times \mathbb{R}^{n-m}$ onto the set $\mathcal{D}^{\prime}=K^{\prime} \times \mathbb{R}^{n-m}$, where $K^{\prime}=U K$ is a cone in $\mathbb{R}^{m}$. Since $A^{\prime \prime}-B^{T} A^{\prime-1} B$ is a symmetric matrix with only positive eigenvalues, there exists an invertible matrix $W$ such that 
$W\left(A^{\prime \prime}-B^{T} A^{\prime-1} B\right) W^{T}=I_{n-m}$. For $\xi^{\prime}=y^{\prime}$ and $\xi^{\prime \prime}=W y^{\prime \prime}$, we get

$$
L_{0}\left(\partial_{x}\right)=\Delta_{\xi^{\prime}}+\Delta_{\xi^{\prime \prime}}
$$

Hence, the equation (7) has the form (8) after the coordinate transformation

$$
\xi^{\prime}=U x^{\prime}, \quad \xi^{\prime \prime}=W\left(x^{\prime \prime}-B^{T} A^{\prime-1} x^{\prime}\right) .
$$

We denote the Green function of the problem $(8)$ by $\tilde{G}_{0}(\xi, \eta, t)$. This means that

$$
\begin{aligned}
& \left(\partial_{t}-\Delta_{\xi}\right) \tilde{G}_{0}(\xi, \eta, t)=\delta(\xi-\eta) \delta(t) \quad \text { for } \xi, \eta \in \mathcal{D}^{\prime}, t \in \mathbb{R} \\
& \tilde{G}_{0}(\xi, \eta, t)=0 \quad \text { for } \xi \in \partial \mathcal{D}^{\prime}, \eta \in \mathcal{D}^{\prime},\left.\quad \tilde{G}_{0}(\xi, \eta, t)\right|_{t<0}=0 .
\end{aligned}
$$

Then the function

$$
G_{0}(x, y, t)=|\operatorname{det} S| \tilde{G}_{0}(S x, S y, t)
$$

is the Green function of the problem (7).

In order to describe the asymptotics of $G_{0}$ near the edge, i.e., for small $\left|x^{\prime}\right|$, we introduce the following notation. We denote by $\left\{\Lambda_{j}\right\}$ the nondecreasing sequence of eigenvalues of the Beltrami operator $-\delta$ on the subdomain $\Omega^{\prime}=K^{\prime} \cap S^{m-1}$ of the unit sphere $S^{m-1}$ with Dirichlet boundary condition, and by $\left\{\phi_{j}\right\}$ an orthonormalized (in $L_{2}\left(\Omega^{\prime}\right)$ ) system of eigenfunction to the eigenvalues $\Lambda_{j}$. Furthermore, let

$$
\lambda_{j}^{ \pm}=\frac{2-m}{2} \pm \frac{1}{2} \sqrt{(2-m)^{2}+4 \Lambda_{j}}
$$

be the solutions of the quadratic equation $\lambda(m-2+\lambda)=\Lambda_{j}$. Then the functions

$$
\tilde{u}_{j}\left(\xi^{\prime}\right)=\left|\xi^{\prime}\right|^{\lambda_{j}^{+}} \phi_{j}\left(\omega_{\xi}\right) \text { and } \quad \tilde{v}_{j}\left(\xi^{\prime}\right)=-\frac{1}{2 \lambda_{j}^{+}+m-2}\left|\xi^{\prime}\right|^{\lambda_{j}^{-}} \phi_{j}\left(\omega_{\xi}\right)
$$

are special solutions of the equation $\Delta_{\xi^{\prime}} u=0$ in $K^{\prime}$. We also introduce the functions

$$
\tilde{w}_{j}\left(\eta^{\prime}, t\right)=\frac{2}{\Gamma\left(\lambda_{j}^{+}+m / 2\right)}(4 t)^{-\lambda_{j}^{+}-m / 2} \tilde{u}_{j}\left(\eta^{\prime}\right) \exp \left(-\frac{\left|\eta^{\prime}\right|^{2}}{4 t}\right),
$$

which are special solutions of the heat equation $\left(\partial_{t}-\Delta_{\eta^{\prime}}\right) \tilde{w}\left(\eta^{\prime}, t\right)=0$ for $\eta^{\prime} \in K^{\prime}$ and $t>0$.

Suppose that $\mu$ is a real number satisfying the inequalities $\lambda_{1}^{+}<\mu<\lambda_{1}^{+}+1$ and $\mu \neq \lambda_{j}^{+}$for all $j$. By [8, Theorem 2.1], the Green function $\tilde{G}_{0}$ admits the decomposition

$$
\begin{aligned}
\tilde{G}_{0}(\xi, \eta, t) & =\Phi\left(\xi^{\prime \prime}-\eta^{\prime \prime}, t\right) \tilde{g}\left(\xi^{\prime}, \eta^{\prime}, t\right) \\
& =\Phi\left(\xi^{\prime \prime}-\eta^{\prime \prime}, t\right)\left(\sum_{\lambda_{j}^{+}<\mu} \tilde{w}_{j}\left(\eta^{\prime}, t\right) \tilde{u}_{j}\left(\xi^{\prime}\right)+\tilde{r}\left(\xi^{\prime}, \eta^{\prime}, t\right)\right),
\end{aligned}
$$

where $\Phi\left(\xi^{\prime \prime}, t\right)=(4 \pi t)^{(m-n) / 2} \exp \left(-\frac{\left|\xi^{\prime \prime}\right|^{2}}{4 t}\right)$ is the fundamental solution of the heat equation in $\mathbb{R}^{n-m}$ and $\tilde{g}\left(\xi^{\prime}, \eta^{\prime}, t\right)$ is the Green function of the Dirichlet problem for the heat equation 
in $K^{\prime}$. The remainder $\tilde{r}\left(\xi^{\prime}, \eta^{\prime}, t\right)$ in (11) satisfies the estimate

$$
\begin{aligned}
\left|\partial_{t}^{k} \partial_{\xi^{\prime}}^{\alpha^{\prime}} \partial_{\eta^{\prime}}^{\gamma^{\prime}} \tilde{r}\left(\xi^{\prime}, \eta^{\prime}, t\right)\right| \leq & c t^{-k-\left(m+\left|\alpha^{\prime}\right|+\left|\gamma^{\prime}\right|\right) / 2}\left(\frac{\left|\xi^{\prime}\right|}{\sqrt{t}}\right)^{\mu-\left|\alpha^{\prime}\right|}\left(\frac{\left|\eta^{\prime}\right|}{\left|\eta^{\prime}\right|+\sqrt{t}}\right)^{\lambda_{1}^{+}-\left|\gamma^{\prime}\right|-\varepsilon} \\
& \times\left(\frac{d\left(\xi^{\prime}\right)}{\left|\xi^{\prime}\right|}\right)^{-\varepsilon_{\alpha^{\prime}}}\left(\frac{d\left(\eta^{\prime}\right)}{\left|\eta^{\prime}\right|}\right)^{-\varepsilon_{\gamma^{\prime}}} \exp \left(-\frac{\kappa\left|\eta^{\prime}\right|^{2}}{t}\right)
\end{aligned}
$$

for $\left|\xi^{\prime}\right|<c \sqrt{t},\left|\alpha^{\prime}\right| \leq 2,\left|\gamma^{\prime}\right| \leq 2$. Here, $\varepsilon_{\alpha^{\prime}}=0$ for $\left|\alpha^{\prime}\right| \leq 1$, while $\varepsilon_{\alpha^{\prime}}$ is an arbitrarily small positive real number if $\left|\alpha^{\prime}\right|=2$.

Using the decomposition (11), we obtain an analogous decomposition for the Green function $G_{0}(x, y, t)$. For this, we introduce the functions

$$
u_{j}\left(x^{\prime}\right)=\tilde{u}_{j}\left(U x^{\prime}\right), \quad v_{j}\left(x^{\prime}\right)=|\operatorname{det} U| \tilde{v}_{j}\left(U x^{\prime}\right)
$$

and

$$
\begin{aligned}
\psi_{j, 0}\left(x^{\prime \prime}, y, t\right) & =|\operatorname{det} S|(4 \pi t)^{(m-n) / 2} \tilde{w}_{j}\left(U y^{\prime}, t\right) \exp \left(-\frac{\left|W\left(x^{\prime \prime}-y^{\prime \prime}+B^{T} A^{\prime-1} y^{\prime}\right)\right|^{2}}{4 t}\right) \\
& =\frac{2 \pi^{(m-n) / 2}(4 t)^{-\lambda_{1}^{+}-n / 2}}{|\operatorname{det} A|^{1 / 2} \Gamma\left(\lambda_{1}^{+}+m / 2\right)} u_{j}\left(y^{\prime}\right) \exp \left(-\frac{q\left(y^{\prime}, x^{\prime \prime}-y^{\prime \prime}\right)}{4 t}\right),
\end{aligned}
$$

where $q\left(y^{\prime}, y^{\prime \prime}\right)$ denotes the quadratic form

$$
q\left(y^{\prime}, y^{\prime \prime}\right)=\left|U y^{\prime}\right|^{2}+\left|W\left(y^{\prime \prime}+B^{T} A^{\prime-1} y^{\prime}\right)\right|^{2} .
$$

Note that the form $q\left(y^{\prime}, y^{\prime \prime}\right)$ is independent of the coordinate transformation since $U^{T} U=$ $A^{\prime-1}$ and $W^{T} W=\left(A^{\prime \prime}-B^{T} A^{\prime-1} B\right)^{-1}$. Since $U$ and $W$ are invertible matrices, there exists a positive constant $\kappa$ such that

$$
q\left(y^{\prime}, y^{\prime \prime}\right) \geq \kappa\left(\left|y^{\prime}\right|^{2}+\left|y^{\prime \prime}\right|^{2}\right) \quad \text { for all } y^{\prime}, y^{\prime \prime} .
$$

We furthermore note that both $u_{j}\left(x^{\prime}\right)$ and $v_{j}\left(x^{\prime}\right)$ are solutions of the equation

$$
L_{0}\left(\partial_{x^{\prime}}, 0\right) u=\sum_{i, j=1}^{m} a_{i j} \partial_{x_{i}} \partial_{x_{j}} u=0 \quad \text { in } K
$$

which have the form

$$
u_{j}\left(x^{\prime}\right)=\left|x^{\prime}\right|^{\lambda_{j}^{+}} \Phi_{j}^{+}\left(\omega_{x}\right), \quad v_{j}\left(x^{\prime}\right)=\left|x^{\prime}\right|^{\lambda_{j}^{-}} \Phi_{j}^{-}\left(\omega_{x}\right) .
$$

This means in particular that $\lambda_{j}^{ \pm}$are eigenvalues and $\Phi_{j}^{ \pm}$are eigenfunctions of the pencil $\mathfrak{A}(\lambda)$ which is defined as

$$
\mathfrak{A}(\lambda) \Phi(\omega)=\left|x^{\prime}\right|^{2-\lambda} L_{0}\left(\partial_{x^{\prime}}, 0\right)\left|x^{\prime}\right|^{\lambda} \Phi(\omega), \quad \Phi \in \stackrel{\circ}{W_{2}^{1}}(\Omega) .
$$

Theorem 2.1 Suppose that $\lambda_{1}^{+}<\mu<\lambda_{1}^{+}+1$ and $\mu \neq \lambda_{j}^{+}$for all $j$. Then the Green function $G_{0}(x, y, t)$ admits the decomposition

$$
G_{0}(x, y, t)=\sum_{\lambda_{j}^{+}<\mu} \psi_{j, 0}\left(x^{\prime \prime}, y, t\right) u_{j}\left(x^{\prime}\right)+R_{0}(x, y, t),
$$


where

$$
\begin{aligned}
\left|\partial_{t}^{k} \partial_{x}^{\alpha} \partial_{y}^{\gamma} R_{0}(x, y, t)\right| \leq & c t^{-k-(n+|\alpha|+|\gamma|) / 2}\left(\frac{\left|x^{\prime}\right|}{\sqrt{t}}\right)^{\mu-\left|\alpha^{\prime}\right|}\left(\frac{\left|y^{\prime}\right|}{\left|y^{\prime}\right|+\sqrt{t}}\right)^{\lambda_{1}^{+}-\left|\gamma^{\prime}\right|-\varepsilon} \\
& \times\left(\frac{d(x)}{\left|x^{\prime}\right|}\right)^{-\varepsilon_{\alpha^{\prime}}}\left(\frac{d(y)}{\left|y^{\prime}\right|}\right)^{-\varepsilon_{\gamma^{\prime}}} \exp \left(-\frac{\kappa\left(\left|y^{\prime}\right|^{2}+\left|x^{\prime \prime}-y^{\prime \prime}\right|^{2}\right)}{t}\right)
\end{aligned}
$$

for $\left|x^{\prime}\right|<\sqrt{t}, \alpha=\left(\alpha^{\prime}, \alpha^{\prime \prime}\right), \gamma=\left(\gamma^{\prime}, \gamma^{\prime \prime}\right),\left|\alpha^{\prime}\right| \leq 2$, and $\left|\gamma^{\prime}\right| \leq 2$. Here, $\varepsilon_{\alpha^{\prime}}=0$ for $\left|\alpha^{\prime}\right| \leq 1$, while $\varepsilon_{\alpha^{\prime}}$ is an arbitrarily small positive real number if $\left|\alpha^{\prime}\right|=2$.

Proof By (10) and (11), we have

$$
\begin{aligned}
G_{0}(x, y, t) & =|\operatorname{det} S| \tilde{G}_{0}(S x, S y, t)=|\operatorname{det} S| \Phi\left(\xi^{\prime \prime}-\eta^{\prime \prime}, t\right) \tilde{g}\left(\xi^{\prime}, \eta^{\prime}, t\right) \\
& =|\operatorname{det} S| \Phi\left(\xi^{\prime \prime}-\eta^{\prime \prime}+W B^{T} A^{\prime-1} x^{\prime}, t\right) \sum_{\lambda_{j}^{+}<\mu} \tilde{w}_{j}\left(\eta^{\prime}, t\right) \tilde{u}_{j}\left(\xi^{\prime}\right)+R_{0}(x, y, t),
\end{aligned}
$$

where $\xi^{\prime}=U x^{\prime}, \eta^{\prime}=U y^{\prime}, \xi^{\prime \prime}=W\left(x^{\prime \prime}-B^{T} A^{\prime-1} x^{\prime}\right)$, and $\eta^{\prime \prime}=W\left(y^{\prime \prime}-B^{T} A^{\prime-1} y^{\prime}\right)$, and

$$
\begin{aligned}
R_{0}(x, y, t)= & |\operatorname{det} S|\left(\Phi\left(\xi^{\prime \prime}-\eta^{\prime \prime}, t\right)-\Phi\left(\xi^{\prime \prime}-\eta^{\prime \prime}+W B^{T} A^{\prime-1} x^{\prime}, t\right)\right) \tilde{g}\left(U x^{\prime}, U y^{\prime}, t\right) \\
& +|\operatorname{det} S| \Phi\left(\xi^{\prime \prime}-\eta^{\prime \prime}+W B^{T} A^{\prime-1} x^{\prime}, t\right) \tilde{r}\left(\xi^{\prime}, \eta^{\prime}, t\right) .
\end{aligned}
$$

The right-hand side of (16) is equal to

$$
\sum_{\lambda_{j}^{+}<\mu} \psi_{j, 0}\left(x^{\prime \prime}, y, t\right) u_{j}\left(x^{\prime}\right)+R_{0}(x, y, t)
$$

Using (12), one can easily check that $R_{0}$ satisfies (15).

We derive another formula for the coefficient $\psi_{j, 0}\left(x^{\prime \prime}, y, t\right)$ in Theorem 2.1. If $t>0$, then

$$
\Delta_{\xi^{\prime}} \tilde{g}\left(\xi^{\prime}, \eta^{\prime}, t\right)=\partial_{t} \tilde{g}\left(\xi^{\prime}, \eta^{\prime}, t\right)
$$

for $\xi^{\prime}, \eta^{\prime} \in K^{\prime}$. Let $V_{p ; \beta}^{l}(K)$ denote the weighted Sobolev space (closure of $C_{0}^{\infty}(\bar{K} \backslash\{0\})$ ) with the norm

$$
\|u\|_{V_{p ; \beta}^{l}(K)}=\left(\int_{K} \sum_{|\alpha| \leq l}\left|x^{\prime}\right|^{p(\beta-l+|\alpha|)}\left|\partial_{x^{\prime}}^{\alpha} u\left(x^{\prime}\right)\right|^{p} d x^{\prime}\right)^{1 / p} .
$$

It follows from (11) and (12) that $\partial_{t} \tilde{g}\left(\cdot, \eta^{\prime}, t\right) \in V_{p ; \beta}^{0}\left(K^{\prime}\right)$ for arbitrary $p$ and $\beta$ such that $p(\beta+$ $\left.\lambda_{1}^{+}\right)>-m$. Hence, the coefficient $\tilde{w}_{j}\left(\eta^{\prime}, t\right)$ in (11) is given by the formula

$$
\tilde{w}_{j}\left(\eta^{\prime}, t\right)=\int_{K^{\prime}} \tilde{v}_{j}\left(\xi^{\prime}\right) \Delta_{\xi^{\prime}} \tilde{g}\left(\xi^{\prime}, \eta^{\prime}, t\right) d \xi^{\prime}
$$

(cf. [14, Theorem 5.1]). Let

$$
U(\xi, \eta, t)=\tilde{g}\left(\xi^{\prime}, \eta^{\prime}, t\right)\left(\Phi\left(\xi^{\prime \prime}-\eta^{\prime \prime}, t\right)-\Phi\left(\xi^{\prime \prime}-\eta^{\prime \prime}-W B^{T} U^{T} \xi^{\prime}, t\right)\right) .
$$


In the integral

$$
\tilde{w}_{j}\left(\eta^{\prime}, t\right)=\int_{K^{\prime}} \tilde{v}_{j}\left(\xi^{\prime}\right) \Delta_{\xi^{\prime}} U(\xi, \eta, t) d \xi^{\prime}
$$

one can integrate by parts for $t>0$. Since $\Delta_{\xi^{\prime}} v_{j}\left(\xi^{\prime}\right)=0$ and $\tilde{g}\left(\xi^{\prime}, \eta^{\prime}, t\right)=v_{j}\left(\xi^{\prime}\right)=0$ for $\xi^{\prime} \in$ $\partial K^{\prime}$, we conclude that the integral (18) vanishes. Hence, it follows from (17) that

$$
\tilde{w}_{j}\left(\eta^{\prime}, t\right) \Phi\left(\xi^{\prime \prime}-\eta^{\prime \prime}, t\right)=\int_{K^{\prime}} \tilde{v}_{j}\left(\xi^{\prime}\right) \Delta_{\xi^{\prime}} \tilde{g}\left(\xi^{\prime}, \eta^{\prime}, t\right) \Phi\left(\xi^{\prime \prime}-\eta^{\prime \prime}-W B^{T} U^{T} \xi^{\prime}, t\right) d \xi^{\prime} .
$$

We set $\xi^{\prime \prime}=W x^{\prime \prime}, \eta^{\prime}=U y^{\prime}, \eta^{\prime \prime}=W\left(y^{\prime \prime}-B^{T} A^{\prime-1} y^{\prime}\right)$, and we substitute $\xi^{\prime}=U x^{\prime}$ in the integral on the right-hand side. Then we obtain

$$
\tilde{w}_{j}\left(U y^{\prime}, t\right) \Phi\left(W\left(x^{\prime \prime}-y^{\prime \prime}+B^{T} A^{\prime-1} y^{\prime}\right), t\right)=\int_{K} v_{j}\left(x^{\prime}\right) L_{0}\left(\partial_{x^{\prime}}, 0\right) \tilde{G}_{0}(S x, S y, t) d x^{\prime} .
$$

Multiplying the last equality by $|\operatorname{det} S|$, we arrive at the formula

$$
\psi_{j, 0}\left(x^{\prime \prime}, y, t\right)=\int_{K} v_{j}\left(x^{\prime}\right) L_{0}\left(\partial_{x^{\prime}}, 0\right) G(x, y, t) d x^{\prime} .
$$

\section{Green function of parabolic equations with variable coefficients}

Now let $L\left(x, t, \partial_{x}\right)$ be the operator (2) with $x$ - and $t$-depending coefficients satisfying the condition (3). We consider the Green function $G(x, y, t, \tau)$ for the operator

$$
\mathcal{L}=\partial_{t}-L\left(x, t, \partial_{x}\right)
$$

in $\mathcal{D}=K \times \mathbb{R}^{n-m}$, i.e., the solution of the problem

$$
\begin{array}{ll}
\left(\partial_{t}-L\left(x, t, \partial_{x}\right)\right) G(x, y, t, \tau)=\delta(x-y) \delta(t-\tau) & \text { for } x, y \in \mathcal{D}, t, \tau \in \mathbb{R}, \\
G(x, y, t, \tau)=0 \quad \text { for } x \in \partial \mathcal{D}, y \in \mathcal{D}, t \in \mathbb{R}, & \left.G(x, y, t, \tau)\right|_{t<\tau}=0 .
\end{array}
$$

In this section, we will employ an estimate for the Green function which was proved in [13]. For this end, we assume in the following that the coefficients of $L$ satisfy some additional smoothness conditions. To be more precise, we suppose that

$$
\partial_{x}^{\gamma} a_{i j} \in C^{\sigma, \sigma / 2}(\mathcal{D} \times \mathbb{R}) \quad \text { for }|\gamma| \leq 2 \quad \text { and } \quad a_{0}, a_{j}, \partial_{x_{i}} a_{j} \in C^{\sigma, \sigma / 2}(\mathcal{D} \times \mathbb{R})
$$

with some $\sigma \in(0,1)$ for $i, j=1, \ldots, n$ and that

$$
\sum_{i, j=1}^{n} \sum_{|\gamma| \leq 2}\left|\partial_{x}^{\gamma} \partial_{x^{\prime \prime}}^{\alpha^{\prime \prime}} \partial_{t}^{k} a_{i j}\right|+\sum_{j=1}^{n} \sum_{|\gamma| \leq 1}\left|\partial_{x}^{\gamma} \partial_{x^{\prime \prime}}^{\alpha^{\prime \prime}} \partial_{t}^{k} a_{j}\right|+\left|\partial_{x^{\prime \prime}}^{\alpha^{\prime \prime}} \partial_{t}^{k} a_{0}\right| \leq C \quad \text { for }\left|\alpha^{\prime \prime}\right| \leq 4, k \leq 2
$$

\subsection{Estimates of Green function}

Let

$$
L_{0}\left(0, x^{\prime \prime}, t, \partial_{x}\right)=\sum_{i, j=1}^{n} a_{i j}\left(0, x^{\prime \prime}, t\right) \partial_{x_{i}} \partial_{x_{j}} \quad \text { and } \quad L_{0}\left(0, x^{\prime \prime}, t, \partial_{x^{\prime}}, 0\right)=\sum_{i, j=1}^{m} a_{i j}\left(0, x^{\prime \prime}, t\right) \partial_{x_{i}} \partial_{x_{j}} \text {. }
$$


Furthermore, let the pencil $\mathfrak{A}\left(x^{\prime \prime}, t ; \lambda\right)$ be defined by $(5)$, and let $\lambda_{j}^{ \pm}\left(x^{\prime \prime}, t\right)$ be its eigenvalues, where

$$
\cdots \leq \lambda_{2}^{-}<\lambda_{1}^{-}<2-m \leq 0<\lambda_{1}^{+}<\lambda_{2}^{+} \leq \cdots .
$$

The following estimate for the Green function $G(x, y, t, \tau)$ is proved in [13, Theorem 4.4].

Theorem 3.1 Suppose that the coefficients of L satisfy the conditions (3), (21), and (22). If $T$ is a positive number and $\lambda<\lambda_{1}^{+}(0,0)-C \sqrt{\epsilon}$, then $G(x, y, t, \tau)$ satisfies the estimate

$$
\begin{aligned}
& \left|\partial_{t}^{k} \partial_{\tau}^{l} \partial_{x}^{\alpha} \partial_{y}^{\gamma} G(x, y, t, \tau)\right| \\
& \leq c(t-\tau)^{-(n+2 k+2 l+|\alpha|+|\gamma|) / 2}\left(\frac{\left|x^{\prime}\right|}{\left|x^{\prime}\right|+\sqrt{t-\tau}}\right)^{\lambda-\left|\alpha^{\prime}\right|}\left(\frac{\left|y^{\prime}\right|}{\left|y^{\prime}\right|+\sqrt{t-\tau}}\right)^{\lambda-\left|\gamma^{\prime}\right|} \\
& \quad \times\left(\frac{d(x)}{\left|x^{\prime}\right|}\right)^{-\varepsilon}\left(\frac{d(y)}{\left|y^{\prime}\right|}\right)^{-\varepsilon} \gamma_{\gamma^{\prime}} \exp \left(-\frac{\kappa|x-y|^{2}}{t-\tau}\right)
\end{aligned}
$$

for $0<t-\tau<T,\left|\alpha^{\prime}\right| \leq 2,\left|\gamma^{\prime}\right| \leq 2,\left|\alpha^{\prime \prime}\right| \leq 4,\left|\gamma^{\prime \prime}\right| \leq 4, k, l \leq 2$. Here, $\varepsilon_{\alpha^{\prime}}$ denotes the same nonnegative number as in Theorem 2.1 .

\subsection{Asymptotics of Green function}

Analogously to the matrix $U$ in Section 2, let $U\left(x^{\prime \prime}, t\right)$ be a matrix such that

$$
U\left(x^{\prime \prime}, t\right) A^{\prime}\left(x^{\prime \prime}, t\right) U^{T}\left(x^{\prime \prime}, t\right)=I_{m},
$$

where $A^{\prime}\left(x^{\prime \prime}, t\right)$ is the matrix with the elements $a_{i j}\left(0, x^{\prime \prime}, t\right), i, j=1, \ldots, m$. Under our assumptions on the coefficients, we may assume that the elements of $U$ are $C^{2}$-functions. Then the numbers $\Lambda_{j}\left(x^{\prime \prime}, t\right)=\lambda_{j}^{+}\left(x^{\prime \prime}, t\right) \lambda_{j}^{-}\left(x^{\prime \prime}, t\right)$ are eigenvalues of the Beltrami operator $-\delta$ (with Dirichlet boundary conditions) on the subdomain $\Omega^{\prime}\left(x^{\prime \prime}, t\right)=K^{\prime}\left(x^{\prime \prime}, t\right) \cap S^{m-1}$ of the unit sphere, where $K^{\prime}\left(x^{\prime \prime}, t\right)=U\left(x^{\prime \prime}, t\right) K$. As in Section 2, we denote by $\left\{\phi_{j}\left(x^{\prime \prime}, t ; \omega\right)\right\}$ an orthonormalized system of eigenfunctions corresponding to the eigenvalues $\Lambda_{j}\left(x^{\prime \prime}, t\right)$. Moreover, we set

$$
\begin{aligned}
& \tilde{u}_{j}\left(x^{\prime \prime}, t ; \xi^{\prime}\right)=\left|\xi^{\prime}\right|^{\lambda_{j}^{+}\left(x^{\prime \prime}, t\right)} \phi_{j}\left(x^{\prime \prime}, t ; \omega_{\xi}\right) \text { and } \\
& \tilde{v}_{j}\left(x^{\prime \prime}, t ; \xi^{\prime}\right)=-\frac{1}{2 \lambda_{j}^{+}+m-2}\left|\xi^{\prime}\right|^{\lambda_{j}^{-}\left(x^{\prime \prime}, t\right)} \phi_{j}\left(x^{\prime \prime}, t ; \omega_{\xi}\right) .
\end{aligned}
$$

Then the functions

$$
u_{j}\left(x^{\prime \prime}, t ; x^{\prime}\right)=\tilde{u}_{j}\left(x^{\prime \prime}, t ; U x^{\prime}\right), \quad v_{j}\left(x^{\prime}\right)=|\operatorname{det} U| \tilde{v}_{j}\left(x^{\prime \prime}, t ; U x^{\prime}\right)
$$

are special solutions of the equation $L_{0}\left(0, x^{\prime \prime}, t, \partial_{x^{\prime}}, 0\right) u\left(x^{\prime}\right)=0$ for $x^{\prime} \in K$. By (20), we have

$$
L_{0}\left(0, x^{\prime \prime}, t, \partial_{x^{\prime}}, 0\right) G(x, y, t, \tau)=\partial_{t} G+\left(L_{0}\left(0, x^{\prime \prime}, t, \partial_{x^{\prime}}, 0\right)-L\left(x, t, \partial_{x}\right)\right) G(x, y, t, \tau)
$$

for $x \in \mathcal{D}, t>\tau$. Suppose that $p$ and $\beta$ are such that

$$
\lambda_{1}^{+}(0,0)<2-\beta-m / p<\lambda_{1}^{+}(0,0)+1-C \sqrt{\epsilon}, \quad 2-\beta-m / p \neq \lambda_{j}^{+}\left(x^{\prime \prime}, t\right) \quad \text { for all } j, x^{\prime \prime}, t,
$$


where $C$ is the same constant as in Theorem 3.1. Then by Theorem 3.1, the right-hand side of (25) belongs to the space $V_{p ; \beta}^{0}(K)$ for arbitrary fixed $x^{\prime \prime} \in \mathbb{R}^{n-m}, y \in \mathcal{D}, t>\tau$. Applying $[15$, Theorem 4.2], we obtain

$$
G\left(x^{\prime}, x^{\prime \prime}, y, t, \tau\right)=\sum_{\lambda_{j}^{+}<2-\beta-m / p} \psi_{j}\left(x^{\prime \prime}, y, t, \tau\right) u_{j}\left(x^{\prime \prime}, t ; x^{\prime}\right)+R(x, y, t, \tau)
$$

where $R\left(\cdot, x^{\prime \prime}, y, t, \tau\right) \in V_{p ; \beta}^{2}(K)$. The coefficients $\psi_{j}\left(x^{\prime \prime}, y, t, \tau\right)$ satisfy the equality $(c f .(19))$

$$
\psi_{j}\left(x^{\prime \prime}, y, t, \tau\right)=\int_{K} v_{j}\left(x^{\prime \prime}, t ; x^{\prime}\right) L_{0}\left(0, x^{\prime \prime}, t, \partial_{x^{\prime}}, 0\right) G(x, y, t, \tau) d x^{\prime}
$$

In the following lemma, we give an estimate of these functions.

Lemma 3.1 Suppose that $\sup \lambda_{j}^{+}\left(x^{\prime \prime}, t\right)<\lambda_{1}^{+}(0)+1-C \sqrt{\epsilon}$, where $C$ is the same constant as in Theorem 3.1. Then the function (26) satisfies the estimate

$$
\begin{aligned}
\left|\partial_{t}^{k} \partial_{\tau}^{l} \partial_{x^{\prime \prime}}^{\alpha^{\prime \prime}} \partial_{y}^{\gamma} \psi_{j}\left(x^{\prime \prime}, y, t, \tau\right)\right| \leq & c(t-\tau)^{-k-l-\left(n+\left|\alpha^{\prime \prime}\right|+|\gamma|+\lambda_{j}^{+}\left(x^{\prime \prime}, t\right)\right) / 2}\left(\frac{\left|y^{\prime}\right|}{\sqrt{t-\tau}}\right)^{\lambda-\left|\gamma^{\prime}\right|}\left(\frac{d(y)}{\left|y^{\prime}\right|}\right)^{-\varepsilon_{\gamma^{\prime}}} \\
& \times \exp \left(-\frac{\kappa\left(\left|y^{\prime}\right|^{2}+\left|x^{\prime \prime}-y^{\prime \prime}\right|^{2}\right)}{t-\tau}\right)
\end{aligned}
$$

for $0<t-\tau<T, k \leq 1, l \leq 1$, and $\left|\alpha^{\prime \prime}\right|,\left|\gamma^{\prime}\right|,\left|\gamma^{\prime \prime}\right| \leq 2$. Here, $\kappa$ is a certain positive number, $\lambda$ is an arbitrary number less than $\lambda_{1}^{+}(0)-C \sqrt{\epsilon}$, and $\varepsilon_{\gamma^{\prime}}$ is the same nonnegative number as in Theorem 2.1.

Proof We define $K_{t}=\left\{x \in K:\left|x^{\prime}\right|<\sqrt{t}\right\}$ for $t>0$. Then

$$
\begin{aligned}
\partial_{t}^{k} \partial_{\tau}^{l} \partial_{x^{\prime \prime}}^{\alpha^{\prime \prime}} \partial_{y}^{\gamma} \psi_{j}\left(x^{\prime \prime}, y, t, \tau\right) \\
=\int_{K_{t-\tau}} \partial_{t}^{k} \partial_{\tau}^{l} \partial_{x^{\prime \prime}}^{\alpha^{\prime \prime}} \partial_{y}^{\gamma} v_{j}\left(x^{\prime \prime}, t ; x^{\prime}\right) L_{0}\left(0, x^{\prime \prime}, t, \partial_{x^{\prime}}, 0\right) G(x, y, t, \tau) d x^{\prime} \\
\quad+\int_{K \backslash K_{t-\tau}} \partial_{t}^{k} \partial_{\tau}^{l} \partial_{x^{\prime \prime}}^{\alpha^{\prime \prime}} \partial_{y}^{\gamma} v_{j}\left(x^{\prime \prime}, t ; x^{\prime}\right) L_{0}\left(0, x^{\prime \prime}, t, \partial_{x^{\prime}}, 0\right) G(x, y, t, \tau) d x^{\prime} .
\end{aligned}
$$

We estimate the first integral on the right-hand side of the last equality using the decomposition (25). Theorem 3.1 yields

$$
\begin{aligned}
& \left|\partial_{t}^{v} \partial_{\tau}^{l} \partial_{x^{\prime \prime}}^{\sigma} \partial_{y}^{\gamma}\left(L_{0}\left(0, x^{\prime \prime}, t, \partial_{x^{\prime}}, 0\right)-L\left(x, t, \partial_{x}\right)\right) G(x, y, t, \tau)\right| \\
& \leq c(t-\tau)^{-v-l-(n+|\gamma|+|\sigma|+\lambda+1) / 2}\left|x^{\prime}\right|^{\lambda-1}\left(\frac{d(x)}{\left|x^{\prime}\right|}\right)^{-\varepsilon}\left(\frac{\left|y^{\prime}\right|}{\left|y^{\prime}\right|+\sqrt{t-\tau}}\right)^{\lambda-\left|\gamma^{\prime}\right|} \\
& \quad \times\left(\frac{d(y)}{\left|y^{\prime}\right|}\right)^{-\varepsilon_{\gamma^{\prime}}} \exp \left(-\frac{\kappa\left(\left|y^{\prime}\right|^{2}+\left|x^{\prime \prime}-y^{\prime \prime}\right|^{2}\right)}{2(t-\tau)}\right)
\end{aligned}
$$

for $\left|x^{\prime}\right|^{2}<t-\tau<T, \sigma \leq \alpha^{\prime \prime}$, and $v \leq k$, where $\varepsilon$ is an arbitrarily small positive number. Furthermore,

$$
\left|\partial_{t}^{k-v} \partial_{x^{\prime \prime}}^{\alpha^{\prime \prime}-\sigma} v_{j}\left(x, t ; x^{\prime}\right)\right| \leq c\left|x^{\prime}\right|^{\lambda_{j}^{-}\left(x^{\prime \prime}, t\right)}\left(1+|\log | x^{\prime}||\right)^{k+\left|\alpha^{\prime \prime}\right|-v-|\sigma|} .
$$


The number $\lambda$ can be chosen such that $\sup \lambda_{j}^{+}\left(x^{\prime \prime}, t\right)<\lambda+1$. Consequently,

$$
\begin{aligned}
& \left|\int_{K_{t-\tau}} \partial_{t}^{k} \partial_{\tau}^{l} \partial_{x^{\prime \prime}}^{\alpha^{\prime \prime}} \gamma_{j}\left(x^{\prime \prime}, t ; x^{\prime}\right) \partial_{y}^{\gamma}\left(L_{0}\left(0, x^{\prime \prime}, t, \partial_{x^{\prime}}, 0\right)-L\left(x, t, \partial_{x}\right)\right) G(x, y, t, \tau) d x^{\prime}\right| \\
& \leq c(t-\tau)^{-k-l-\left(n+\left|\alpha^{\prime \prime}\right|+|\gamma|+\lambda+1\right) / 2}\left(\frac{\left|y^{\prime}\right|}{\left|y^{\prime}\right|+\sqrt{t-\tau}}\right)^{\lambda-\left|\gamma^{\prime}\right|} \\
& \quad \times\left(\frac{d(y)}{\left|y^{\prime}\right|}\right)^{-\varepsilon_{\gamma^{\prime}}} \exp \left(-\frac{\kappa\left(\left|y^{\prime}\right|^{2}+\left|x^{\prime \prime}-y^{\prime \prime}\right|^{2}\right)}{2(t-\tau)}\right) \\
& \quad \times \int_{K_{t-\tau}}\left|x^{\prime}\right|^{\lambda-\lambda_{j}^{+}\left(x^{\prime \prime}, t\right)+1-m}\left(1+|\log | x^{\prime}||\right)^{l+\left|\alpha^{\prime \prime}\right|}\left(\frac{d(x)}{\left|x^{\prime}\right|}\right)^{-\varepsilon} d x^{\prime} \\
& \leq c(t-\tau)^{-k-l-\left(n+\left|\alpha^{\prime \prime}\right|+|\gamma|+\lambda_{j}^{+}\left(x^{\prime \prime}, t\right)\right) / 2}\left(\frac{\left|y^{\prime}\right|}{\sqrt{t-\tau}}\right)^{\lambda-\left|\gamma^{\prime}\right|} \\
& \quad \times\left(\frac{d(y)}{\left|y^{\prime}\right|}\right)^{-\varepsilon_{\gamma^{\prime}}} \exp \left(-\frac{\kappa\left(\left|y^{\prime}\right|^{2}+\left|x^{\prime \prime}-y^{\prime \prime}\right|^{2}\right)}{4(t-\tau)}\right) .
\end{aligned}
$$

The same estimate holds for

$$
\left|\int_{K_{t-\tau}} \partial_{t}^{k} \partial_{\tau}^{l} \partial_{x^{\prime \prime}}^{\alpha^{\prime \prime}} v_{j}\left(x^{\prime \prime}, t ; x^{\prime}\right) \partial_{t} \partial_{y}^{\gamma} G(x, y, t, \tau) d x^{\prime}\right|
$$

Thus, we obtain

$$
\begin{aligned}
& \left|\int_{K_{t-\tau}} \partial_{t}^{k} \partial_{x^{\prime \prime}}^{\alpha^{\prime \prime}} v_{j}\left(x^{\prime \prime}, t ; x^{\prime}\right) L_{0}\left(0, x^{\prime \prime}, t, \partial_{x^{\prime}}, 0\right) \partial_{\tau}^{l} \partial_{y}^{\gamma} G(x, y, t, \tau) d x^{\prime}\right| \\
& \leq c(t-\tau)^{-k-l-\left(n+\left|\alpha^{\prime \prime}\right|+|\gamma|+\lambda_{j}^{+}\left(x^{\prime \prime}, t\right)\right) / 2}\left(\frac{\left|y^{\prime}\right|}{\sqrt{t-\tau}}\right)^{\lambda-\left|\gamma^{\prime}\right|} \\
& \quad \times\left(\frac{d(y)}{\left|y^{\prime}\right|}\right)^{-\varepsilon_{\gamma^{\prime}}} \exp \left(-\frac{\kappa\left(\left|y^{\prime}\right|^{2}+\left|x^{\prime \prime}-y^{\prime \prime}\right|^{2}\right)}{4(t-\tau)}\right) .
\end{aligned}
$$

Since $L_{0}\left(0, x^{\prime \prime}, t, \partial_{x^{\prime}}, 0\right) v_{j}\left(x^{\prime \prime}, t ; x^{\prime}\right)=0$ for $x^{\prime} \in K$ and $\partial_{t}^{k} \partial_{\tau}^{l} \partial_{x^{\prime \prime}}^{\alpha^{\prime \prime}} \partial_{y}^{\gamma} G(x, y, t, \tau)$ is exponentially decaying for large $|x|$, we get

$$
\begin{aligned}
& \int_{K \backslash K_{t-\tau}} \partial_{t}^{k} \partial_{x^{\prime \prime}}^{\alpha^{\prime \prime}} v_{j}\left(x^{\prime \prime}, t ; x^{\prime}\right) L_{0}\left(0, x^{\prime \prime}, t, \partial_{x^{\prime}}, 0\right) \partial_{\tau}^{l} \partial_{y}^{\gamma} G(x, y, t, \tau) d x \\
& =\int_{S_{t-\tau}} \partial_{t}^{k} \partial_{x^{\prime \prime}}^{\alpha^{\prime \prime}} \sum_{i, j=1}^{n} a_{i, j}\left(0, x^{\prime \prime}, t\right) \\
& \quad \times\left(v_{j}\left(x^{\prime \prime}, t ; x^{\prime}\right) \partial_{x_{j}} \partial_{\tau}^{l} \partial_{y}^{\gamma} G(x, y, t, \tau)-\partial_{\tau}^{l} \partial_{y}^{\gamma} G(x, y, t, \tau) \partial_{x_{j}} v_{j}\left(x^{\prime \prime}, t ; x^{\prime}\right)\right) \cos \left(\mathbf{n}, x_{j}\right) d \sigma,
\end{aligned}
$$

where $S_{t-\tau}$ is the intersection of $K$ with the sphere $\left|x^{\prime}\right|=\sqrt{t-\tau}$ and $\mathbf{n}$ is the normal vector to this sphere. By Theorem 3.1, the integrand on the right-hand side of the last equality has the upper bound

$$
\begin{gathered}
c(t-\tau)^{-k-l-\left(n+\left|\alpha^{\prime \prime}\right|+|\gamma|+1-\lambda_{j}^{-}(t)\right) / 2}\left(\frac{\left|y^{\prime}\right|}{\left|y^{\prime}\right|+\sqrt{t-\tau}}\right)^{\lambda-\left|\gamma^{\prime}\right|} \\
\times\left(\frac{d(y)}{\left|y^{\prime}\right|}\right)^{-\varepsilon} \exp \left(-\frac{\kappa\left(\left|y^{\prime}\right|^{2}+\left|x^{\prime \prime}-y^{\prime \prime}\right|^{2}\right)}{t-\tau}\right) .
\end{gathered}
$$


Therefore,

$$
\begin{aligned}
& \left|\int_{K \backslash K_{t-\tau}} \partial_{t}^{k} \partial_{x^{\prime \prime}}^{\alpha^{\prime \prime}} v_{j}\left(x^{\prime \prime}, t ; x^{\prime}\right) L_{0}\left(0, x^{\prime \prime}, t, \partial_{x^{\prime}}\right) \partial_{\tau}^{l} \partial_{y}^{\gamma} G(x, y, t, \tau) d x^{\prime}\right| \\
& \leq c(t-\tau)^{-k-l-\left(n+\left|\alpha^{\prime \prime}\right|+|\gamma|+\lambda_{j}^{+}\left(x^{\prime \prime}, t\right)\right) / 2}\left(\frac{\left|y^{\prime}\right|}{\sqrt{t-\tau}}\right)^{\lambda-\left|\gamma^{\prime}\right|} \\
& \quad \times\left(\frac{d(y)}{\left|y^{\prime}\right|}\right)^{-\varepsilon} \exp \left(-\frac{\kappa\left(\left|y^{\prime}\right|^{2}+\left|x^{\prime \prime}-y^{\prime \prime}\right|^{2}\right)}{2(t-\tau)}\right) .
\end{aligned}
$$

This proves the lemma.

For the estimation of the remainder $R(x, y, t, \tau)$, we need the following lemma.

Lemma 3.2 Suppose that $u \in V_{p ; \beta}^{2}(K)$ is a solution of the problem $L_{0}\left(0, x^{\prime \prime}, t, \partial_{x^{\prime}}, 0\right) u=f$ in $K, u=0$ on $\partial K$, where $\lambda_{j}^{+}\left(x^{\prime \prime}, t\right)+\varepsilon<2-\beta-m / p<\lambda_{j+1}^{+}\left(x^{\prime \prime}, t\right)-\varepsilon$ for a certain integer $j \geq 1$. Then

$$
\|u\|_{V_{p ; \beta}^{2}(K)} \leq \frac{c\left(x^{\prime \prime}, t\right)}{\varepsilon}\|f\|_{V_{p ; \beta}^{0}(K)}
$$

with a constant $c\left(x^{\prime \prime}, t\right)$ independent of $\varepsilon$.

Proof First note that the eigenvalues $\lambda_{j}^{+}\left(x^{\prime \prime}, t\right)$ and $\lambda_{j+1}^{+}\left(x^{\prime \prime}, t\right)$ of the pencil $\mathfrak{A}\left(x^{\prime \prime}, t ; \lambda\right)$ have no generalized eigenfunctions (see, e.g., [16, Section 2.2]). Let $g\left(x^{\prime}, y^{\prime}\right)$ be the Green function of the Dirichlet problem for the operator $L_{0}\left(0, x^{\prime \prime}, t, \partial_{x^{\prime}}, 0\right)$ in the cone $K, \zeta g\left(\cdot, y^{\prime}\right) \in V_{p ; \beta}^{2}(K)$ for smooth $\zeta$ vanishing in a neighborhood of $y^{\prime}$. Then

$$
u\left(x^{\prime}\right)=\int_{K} g\left(x^{\prime}, y^{\prime}\right) f\left(y^{\prime}\right) d y^{\prime} .
$$

By [17, Theorem 2.2], the function $g$ satisfies the estimates

$$
\begin{aligned}
& \left|g\left(x^{\prime}, y^{\prime}\right)\right| \leq c\left|x^{\prime}\right|_{j+1}^{\lambda_{j+1}^{+}\left(x^{\prime \prime}, t\right)}\left|y^{\prime}\right|^{2-n-\lambda_{j+1}^{+}(t)} \quad \text { for } 2\left|x^{\prime}\right|<\left|y^{\prime}\right|, \\
& \left|g\left(x^{\prime}, y^{\prime}\right)\right| \leq c\left|x^{\prime}\right|^{\lambda_{j}^{+}\left(x^{\prime \prime}, t\right)}\left|y^{\prime}\right|^{2-n-\lambda_{j}^{+}(t)} \quad \text { for }\left|x^{\prime}\right|>2\left|y^{\prime}\right| .
\end{aligned}
$$

Moreover, in the case $\left|y^{\prime}\right|<2\left|x^{\prime}\right|<4\left|y^{\prime}\right|$, the estimates $\left|g\left(x^{\prime}, y^{\prime}\right)\right| \leq c\left|x^{\prime}-y^{\prime}\right|^{2-m}$ for $m>2$ and $\left|g\left(x^{\prime}, y^{\prime}\right)\right| \leq c|\log | x^{\prime}-y^{\prime}||$ for $m=2$ are valid. For arbitrary integer $v$, let $\chi_{v}\left(x^{\prime}\right)=1$ for $2^{v-1} \leq\left|x^{\prime}\right| \leq 2^{v}, \chi_{v}\left(x^{\prime}\right)=0$ else. Furthermore, let

$$
u_{v}\left(x^{\prime}\right)=\int_{K} g\left(x^{\prime}, y^{\prime}\right) \chi_{v}\left(y^{\prime}\right) f\left(y^{\prime}\right) d y^{\prime}
$$

Then it follows from the above estimates for $g\left(x^{\prime}, y^{\prime}\right)$ and from [18, Lemmas 3.5.1 and 3.5.4] that

$$
\begin{array}{ll}
\left\|\chi_{\mu} u_{v}\right\|_{V_{p ; \beta-2}^{0}(K)} \leq c 2^{(\mu-v)\left(\lambda_{j}^{+}\left(x^{\prime \prime}, t\right)-2+\beta+m / p\right)}\left\|\chi_{\nu} f\right\|_{V_{p ; \beta}^{0}(K)} \quad \text { if } \mu \geq v, \\
\left\|\chi_{\mu} u_{v}\right\|_{V_{p ; \beta-2}^{0}(K)} \leq c 2^{(\mu-v)\left(\lambda_{j+1}^{+}\left(x^{\prime \prime}, t\right)-2+\beta+m / p\right)}\left\|\chi_{\nu} f\right\|_{V_{p ; \beta}^{0}(K)} \quad \text { if } \mu<v,
\end{array}
$$


Kozlov and Rossmann Boundary Value Problems 2014, 2014:252

Page 13 of 37

http://www.boundaryvalueproblems.com/content/2014/1/252

where $c$ is independent of $\mu, v$, and $f(c f$. [18, Lemma 3.5.6]). Consequently,

$$
\begin{aligned}
\|u\|_{V_{p ; \beta-2}^{0}(K)}^{p} & =\sum_{\mu}\left\|\chi_{\mu} u\right\|_{V_{p ; \beta-2}^{0}(K)}^{p} \leq \sum_{\mu}\left(\sum_{\nu}\left\|\chi_{\mu} u_{\nu}\right\|_{V_{p ; \beta-2}^{0}(K)}\right)^{p} \\
& \leq c \sum_{\mu}\left(\sum_{\nu} 2^{-\varepsilon|\mu-\nu|}\left\|\chi_{\nu} f\right\|_{V_{p ; \beta}^{0}(K)}\right)^{p}
\end{aligned}
$$

By Hölder's inequality,

$$
\begin{aligned}
\left(\sum_{\nu} 2^{-\varepsilon|\mu-\nu|}\left\|\chi_{\nu} f\right\|_{V_{p ; \beta}^{0}(K)}\right)^{p} & \leq\left(\sum_{\nu} 2^{-\varepsilon|\mu-\nu|}\left\|\chi_{\nu} f\right\|_{V_{p ; \beta}^{0}(K)}^{p}\right)\left(\sum_{\nu} 2^{-\varepsilon|\mu-\nu|}\right)^{p-1} \\
& =\left(\frac{2^{\varepsilon}+1}{2^{\varepsilon}-1}\right)^{p-1} \sum_{\nu} 2^{-\varepsilon|\mu-\nu|}\left\|\chi_{\nu} f\right\|_{V_{p ; \beta}^{0}(K)}^{p}
\end{aligned}
$$

Thus, we obtain

$$
\begin{aligned}
\|u\|_{V_{p ; \beta-2}^{0}(K)}^{p} & \leq c\left(\frac{2^{\varepsilon}+1}{2^{\varepsilon}-1}\right)^{p-1} \sum_{\mu} \sum_{\nu} 2^{-\varepsilon|\mu-\nu|}\left\|\chi_{\nu} f\right\|_{V_{p ; \beta}^{0}(K)}^{p} \\
& =c\left(\frac{2^{\varepsilon}+1}{2^{\varepsilon}-1}\right)^{p} \sum_{\nu}\left\|\chi_{\nu} f\right\|_{V_{p ; \beta}^{0}(K)}^{p}=c\left(\frac{2^{\varepsilon}+1}{2^{\varepsilon}-1}\right)^{p}\|f\|_{V_{p ; \beta}^{0}(K)^{*}}^{p}
\end{aligned}
$$

The last inequality together with the estimate

$$
\|u\|_{V_{p ; \beta}^{2}(K)} \leq c\left(\|f\|_{V_{p ; \beta}^{0}(K)}+\|u\|_{V_{p ; \beta-2}^{0}(K)}\right)
$$

(see, e.g., [18, Theorem 3.3.5]) implies (28).

Now we are able to prove the main result of this section.

Theorem 3.2 Suppose that $\lambda<\lambda_{1}^{+}(0)-C \sqrt{\epsilon}$ and

$$
\lambda_{1}^{+}(0)<\mu<\lambda_{1}^{+}(0)+1-C \sqrt{\epsilon}, \quad \lambda_{j}^{+}\left(x^{\prime \prime}, t\right) \notin[\mu-\sqrt{\epsilon}, \mu+\sqrt{\epsilon}] \text { for all } j, x^{\prime \prime}, t,
$$

where $C$ is the same constant as in Theorem 3.1. Then the Green function $G(x, y, t, \tau)$ admits the decomposition

$$
G(x, y, t, \tau)=\sum_{\lambda_{j}^{+}<\mu} \psi_{j}\left(x^{\prime \prime}, y, t, \tau\right) u_{j}\left(x^{\prime \prime}, t ; x^{\prime}\right)+R(x, y, t, \tau),
$$

where $u_{j}$ is defined by (24), and

$$
\begin{aligned}
\left|\partial_{t}^{k} \partial_{\tau}^{l} \partial_{x}^{\alpha} \partial_{y}^{\gamma} R(x, y, t, \tau)\right| \leq & c(t-\tau)^{-k-l-(n+|\alpha|+|\gamma|) / 2}\left(\frac{\left|x^{\prime}\right|}{\sqrt{t-\tau}}\right)^{\mu-\left|\alpha^{\prime}\right|}\left(\frac{\left|y^{\prime}\right|}{\sqrt{t-\tau}}\right)^{\lambda-\left|\gamma^{\prime}\right|} \\
& \times\left(\frac{d(y)}{|y|}\right)^{-\varepsilon \gamma^{\prime}} \exp \left(-\frac{\kappa\left(\left|y^{\prime}\right|^{2}+\left|x^{\prime \prime}-y^{\prime \prime}\right|^{2}\right)}{t-\tau}\right)
\end{aligned}
$$


for $0<t-\tau<T,\left|x^{\prime}\right|<\sqrt{t-\tau},\left|\alpha^{\prime}\right| \leq 1,\left|\alpha^{\prime \prime}\right|,\left|\gamma^{\prime}\right|,\left|\gamma^{\prime \prime}\right| \leq 2, k, l \leq 1$. Here, $\varepsilon_{\gamma^{\prime}}$ is the same constant as in Theorem 2.1. The coefficients $\psi_{j}\left(x^{\prime \prime}, y, t, \tau\right)$ satisfy the estimate (27).

Proof Let $\zeta$ be a smooth function on the interval $(0, \infty), \zeta(r)=1$ for $r<1$ and $\zeta(r)=0$ for $r>2$. Furthermore, let $\chi\left(x^{\prime}, t\right)=\zeta\left(t^{-1 / 2}\left|x^{\prime}\right|\right)$ for $x=\left(x^{\prime}, x^{\prime \prime}\right) \in \mathcal{D}$ and $t>0$. It follows from the equality

$$
\begin{aligned}
L_{0}\left(0, x^{\prime \prime}, t, \partial_{x^{\prime}}, 0\right) R(x, y, t, \tau)= & L_{0}\left(0, x^{\prime \prime}, t, \partial_{x^{\prime}}, 0\right) G(x, y, t, \tau) \\
= & \left(L_{0}\left(0, x^{\prime \prime}, t, \partial_{x^{\prime}}, 0\right)-L\left(x, t, \partial_{x}\right)\right) G(x, y, t, \tau) \\
& +\partial_{t} G(x, y, t, \tau)
\end{aligned}
$$

that

$$
L_{0}\left(0, x^{\prime \prime}, t, \partial_{x^{\prime}}, 0\right) \chi\left(x^{\prime}, t-\tau\right) \partial_{\tau}^{l} \partial_{y}^{\gamma} R(x, y, t, \tau)=f(x, y, t, \tau)
$$

for $t>\tau$, where

$$
\begin{aligned}
f= & \chi\left(x^{\prime}, t-\tau\right) \partial_{\tau}^{l} \partial_{y}^{\gamma}\left(\left(L_{0}\left(0, x^{\prime \prime}, t, \partial_{x^{\prime}}, 0\right)-L\left(x, t, \partial_{x}\right)\right) G(x, y, t, \tau)+\partial_{t} G(x, y, t, \tau)\right) \\
& +\left[L_{0}\left(0, x^{\prime \prime}, t, \partial_{x^{\prime}}, 0\right), \chi\left(x^{\prime}, t-\tau\right)\right] \partial_{\tau}^{l} \partial_{y}^{\gamma}\left(G(x, y, t, \tau)-\sum \psi_{j}\left(x^{\prime \prime}, y, t, \tau\right) u_{j}\left(x^{\prime \prime}, t ; x^{\prime}\right)\right) .
\end{aligned}
$$

Here, $\left[L_{0}, \chi\right]=L_{0} \chi-\chi L_{0}$ denotes the commutator of $L_{0}$ and $\chi$. Furthermore, $\partial_{\tau}^{l} \partial_{y}^{\gamma} R(x, y$, $t, \tau)=0$ for $x^{\prime} \in \partial K$. We estimate the $V_{p ; \beta}^{2}(K)$-norm of the function $\chi(\cdot, t-\tau) \partial_{\tau}^{l} \partial_{y}^{\gamma} R\left(\cdot, x^{\prime \prime}, y\right.$, $t, \tau)$ for $2-\beta-m / p=\mu$. By [15, Theorem 4.1],

$$
\left\|\chi(\cdot, t-\tau) \partial_{\tau}^{l} \partial_{y}^{\gamma} R\left(\cdot, x^{\prime \prime}, y, t, \tau\right)\right\|_{V_{p ; \beta}^{2}(K)} \leq c\left\|f\left(\cdot, x^{\prime \prime}, y, t, \tau\right)\right\|_{V_{p ; \beta}^{0}(K)} .
$$

Here, the constant $c$ is independent of $x^{\prime \prime}, y, t, \tau$. Indeed, by Lemma 3.2, we have

$$
\begin{aligned}
& \left\|\chi(\cdot, t-\tau) \partial_{\tau}^{l} \partial_{y}^{\gamma} R\left(\cdot, x^{\prime \prime}, y, t, \tau\right)\right\|_{V_{p ; \beta}^{2}(K)} \\
& \quad \leq \frac{c}{\sqrt{\epsilon}}\left\|L_{0}\left(0,0, \partial_{x^{\prime}}, 0\right) \chi(\cdot, t-\tau) \partial_{\tau}^{l} \partial_{y}^{\gamma} R\left(\cdot, x^{\prime \prime}, y, t, \tau\right)\right\|_{V_{p ; \beta}^{0}(K)},
\end{aligned}
$$

with a constant $c$ independent of $x^{\prime \prime}, y, t, \tau$. Furthermore, under the condition (3), the inequality

$$
\begin{aligned}
& \left\|\left(L_{0}\left(0, x^{\prime \prime}, t, \partial_{x^{\prime}}, 0\right)-L_{0}\left(0,0, \partial_{x^{\prime}}, 0\right)\right) \chi(\cdot, t-\tau) \partial_{\tau}^{l} \partial_{y}^{\gamma} R\left(\cdot, x^{\prime \prime}, y, t, \tau\right)\right\|_{V_{p ; \beta}^{0}(K)} \\
& \quad \leq c \epsilon\left\|\chi(\cdot, t-\tau) \partial_{\tau}^{l} \partial_{y}^{\gamma} R\left(\cdot, x^{\prime \prime}, y, t, \tau\right)\right\|_{V_{p ; \beta}^{2}(K)}
\end{aligned}
$$

holds. Thus,

$$
\begin{aligned}
& \left\|\chi(\cdot, t-\tau) \partial_{\tau}^{l} \partial_{y}^{\gamma} R\left(\cdot, x^{\prime \prime}, y, t, \tau\right)\right\|_{V_{p ; \beta}^{2}(K)} \\
& \quad \leq c_{1} \sqrt{\epsilon}\left\|\chi(\cdot, t-\tau) \partial_{\tau}^{l} \partial_{y}^{\gamma} R\left(\cdot, x^{\prime \prime}, y, t, \tau\right)\right\|_{V_{p ; \beta}^{2}(K)}+\frac{c_{2}}{\sqrt{\epsilon}}\left\|f\left(\cdot, x^{\prime \prime}, y, t, \tau\right)\right\|_{V_{p ; \beta}^{0}(K)},
\end{aligned}
$$


which implies (32) if $\epsilon$ is sufficiently small. Next, we estimate the $V_{p ; \beta}^{0}$-norm of $f\left(\cdot, x^{\prime \prime}, y, t\right)$. By Theorem 3.1,

$$
\begin{gathered}
\left|\chi\left(x^{\prime}, t-\tau\right) \partial_{\tau}^{l} \partial_{y}^{\gamma}\left(L_{0}\left(0, x^{\prime \prime}, t, \partial_{x^{\prime}}, 0\right)-L\left(x, t, \partial_{x}\right)\right) G(x, y, t, \tau)\right| \\
\leq c(t-\tau)^{-l-(n+|\gamma|+\lambda+1) / 2}\left|x^{\prime}\right|^{\lambda-1}\left(\frac{d(x)}{\left|x^{\prime}\right|}\right)^{-\varepsilon}\left(\frac{\left|y^{\prime}\right|}{\sqrt{t-\tau}}\right)^{\lambda-\left|\gamma^{\prime}\right|} \\
\quad \times\left(\frac{d(y)}{\left|y^{\prime}\right|}\right)^{-\varepsilon_{\gamma^{\prime}}} \exp \left(-\frac{\kappa\left(\left|y^{\prime}\right|^{2}+\left|x^{\prime \prime}-y^{\prime \prime}\right|^{2}\right)}{t-\tau}\right)
\end{gathered}
$$

for $0<t-\tau<T$, where $\lambda<\lambda_{1}^{+}(0)-C \sqrt{\epsilon}$. Here, $\lambda$ can be chosen such that $p(\beta+\lambda-1)>-m$. Therefore,

$$
\begin{aligned}
& \left\|\chi(\cdot, t-\tau) \partial_{\tau}^{l} \partial_{y}^{\gamma}\left(L_{0}\left(0, x^{\prime \prime}, t, \partial_{x^{\prime}}, 0\right)-L\left(\cdot, x^{\prime \prime}, t, \partial_{x}\right)\right) G\left(\cdot, x^{\prime \prime}, y, t, \tau\right)\right\|_{V_{p ; \beta}^{0}(K)} \\
& \leq c(t-\tau)^{-l+(\beta-n-|\gamma|-2+m / p) / 2}\left(\frac{\left|y^{\prime}\right|}{\sqrt{t-\tau}}\right)^{\lambda-\left|\gamma^{\prime}\right|} \\
& \quad \times\left(\frac{d(y)}{\left|y^{\prime}\right|}\right)^{-\varepsilon_{\gamma^{\prime}}} \exp \left(-\frac{\kappa\left(\left|y^{\prime}\right|^{2}+\left|x^{\prime \prime}-y^{\prime \prime}\right|^{2}\right)}{t-\tau}\right) .
\end{aligned}
$$

Analogously, we obtain

$$
\begin{aligned}
& \left\|\chi(\cdot, t-\tau) \partial_{t} \partial_{\tau}^{l} \partial_{y}^{\gamma} G\left(\cdot, x^{\prime \prime}, y, t\right)\right\|_{V_{p ; \beta}^{0}(K)} \\
& \leq c(t-\tau)^{-l+(\beta-n-|\gamma|-2+m / p) / 2}\left(\frac{\left|y^{\prime}\right|}{\sqrt{t-\tau}}\right)^{\lambda-\left|\gamma^{\prime}\right|} \\
& \quad \times\left(\frac{d(y)}{\left|y^{\prime}\right|}\right)^{-\varepsilon_{\gamma^{\prime}}} \exp \left(-\frac{\kappa\left(\left|y^{\prime}\right|^{2}+\left|x^{\prime \prime}-y^{\prime \prime}\right|^{2}\right)}{t-\tau}\right) .
\end{aligned}
$$

Since $\left[L_{0}\left(0, x^{\prime \prime}, t, \partial_{x}\right), \chi\left(x^{\prime}, t-\tau\right)\right] G(x, y, t, \tau)$ vanishes for $\left|x^{\prime}\right|<\sqrt{t-\tau}$ and $\left|x^{\prime}\right|>2 \sqrt{t-\tau}$, we obtain the estimate

$$
\begin{aligned}
& \left\|\left[L_{0}\left(0, x^{\prime \prime}, t, \partial_{x^{\prime}}, 0\right), \chi\left(x^{\prime}, t-\tau\right)\right] \partial_{y}^{\gamma} G\left(\cdot, x^{\prime \prime}, y, t, \tau\right)\right\|_{V_{p ; \beta}^{0}(K)} \\
& \leq c(t-\tau)^{-l+(\beta-n-|\gamma|-2+m / p) / 2}\left(\frac{\left|y^{\prime}\right|}{\sqrt{t-\tau}}\right)^{\lambda-\left|\gamma^{\prime}\right|} \\
& \quad \times\left(\frac{d(y)}{|y|}\right)^{-\varepsilon_{\gamma^{\prime}}} \exp \left(-\frac{\kappa\left(\left|y^{\prime}\right|^{2}+\left|x^{\prime \prime}-y^{\prime \prime}\right|^{2}\right)}{t-\tau}\right)
\end{aligned}
$$

by means of Theorem 3.1. Using Lemma 3.1, we get the same estimate for the $V_{p ; \beta}^{0}(K)$ norm of the functions $\left[L_{0}\left(0, x^{\prime \prime}, t, \partial_{x^{\prime}}, 0\right), \chi(\cdot, t-\tau)\right] \partial_{\tau}^{l} \partial_{y}^{\gamma} \psi_{j}\left(x^{\prime \prime}, y, t, \tau\right) u_{j}\left(x^{\prime \prime}, t ; \cdot\right)$. Consequently, (32) implies

$$
\begin{aligned}
& \left\|\chi(\cdot, t-\tau) \partial_{\tau}^{l} \partial_{y}^{\gamma} R\left(\cdot, x^{\prime \prime}, y, t, \tau\right)\right\|_{V_{p ; \beta}^{2}(K)} \\
& \leq c(t-\tau)^{-l+(\beta-n-|\gamma|-2+m / p) / 2}\left(\frac{\left|y^{\prime}\right|}{\sqrt{t-\tau}}\right)^{\lambda-\left|\gamma^{\prime}\right|} \\
& \quad \times\left(\frac{d(y)}{\left|y^{\prime}\right|}\right)^{-\varepsilon} \exp \left(-\frac{\kappa\left(\left|y^{\prime}\right|^{2}+\left|x^{\prime \prime}-y^{\prime \prime}\right|^{2}\right)}{t-\tau}\right)
\end{aligned}
$$


for $0<t-\tau<T$. We prove an analogous estimate for the $x^{\prime \prime}$ - and $t$-derivatives of $\partial_{\tau}^{l} \partial_{y}^{\gamma} R$. Obviously,

$$
\begin{aligned}
& L_{0}\left(0, x^{\prime \prime}, t, \partial_{x^{\prime}}, 0\right)\left(\chi\left(x^{\prime}, t-\tau\right) \partial_{x_{j}} \partial_{\tau}^{l} \partial_{y}^{\gamma} R(x, y, t, \tau)\right) \\
& \quad=\partial_{x_{j}} f(x, y, t, \tau)-\left(\partial_{x_{j}} L_{0}\left(0, x^{\prime \prime}, t, \partial_{x^{\prime}}, 0\right)\right)\left(\chi\left(x^{\prime}, t-\tau\right) \partial_{\tau}^{l} \partial_{y}^{\gamma} R(x, y, t, \tau)\right)
\end{aligned}
$$

for $j \geq m+1$, where $f$ is the same function as above. Since, moreover, $\partial_{x_{j}} \partial_{\tau}^{l} \partial_{y}^{\gamma} R(x, y, t, \tau)=0$ for $x^{\prime} \in \partial K$ and $j \geq m+1$, we get

$$
\begin{aligned}
& \left\|\chi(\cdot, t-\tau) \partial_{x_{j}} \partial_{\tau}^{l} \partial_{y}^{\gamma} R\left(\cdot, x^{\prime \prime}, y, t, \tau\right)\right\|_{V_{p ; \beta}^{2}(K)} \\
& \quad \leq c\left(\left\|\partial_{x_{j}} f\right\|_{V_{p ; \beta}^{0}(K)}+\left\|\chi(\cdot, t-\tau) \partial_{\tau}^{l} \partial_{y}^{\gamma} R\left(\cdot, x^{\prime \prime}, y, t, \tau\right)\right\|_{V_{p ; \beta}^{2}(K)}\right)
\end{aligned}
$$

for $j \geq m+1$. The $V_{p ; \beta}^{0}(K)$-norms of $\partial_{x_{j}} f$ can be estimated in the same way as $f$. This together with (33) leads to the estimate

$$
\begin{aligned}
& \left\|\chi(\cdot, t-\tau) \partial_{x_{j}} \partial_{\tau}^{l} \partial_{y}^{\gamma} R\left(\cdot, x^{\prime \prime}, y, t, \tau\right)\right\|_{V_{p ; \beta}^{2}(K)} \\
& \leq c(t-\tau)^{-l+(\beta-n-|\gamma|-3+m / p) / 2}\left(\frac{\left|y^{\prime}\right|}{\sqrt{t-\tau}}\right)^{\lambda-\left|\gamma^{\prime}\right|} \\
& \quad \times\left(\frac{d(y)}{\left|y^{\prime}\right|}\right)^{-\varepsilon} \gamma^{\prime} \exp \left(-\frac{\kappa\left(\left|y^{\prime}\right|^{2}+\left|x^{\prime \prime}-y^{\prime \prime}\right|^{2}\right)}{t-\tau}\right)
\end{aligned}
$$

for $j \geq m+1$. Analogously, the inequality

$$
\begin{gathered}
\left\|\chi(\cdot, t-\tau) \partial_{t}^{k} \partial_{x^{\prime \prime}}^{\alpha^{\prime \prime}} \partial_{\tau}^{l} \partial_{y}^{\gamma} R\left(\cdot, x^{\prime \prime}, y, t, \tau\right)\right\|_{V_{p ; \beta}^{2}(K)} \\
\leq c(t-\tau)^{-k-l+\left(\beta-n-\left|\alpha^{\prime \prime}\right|-|\gamma|-2+m / p\right) / 2}\left(\frac{\left|y^{\prime}\right|}{\sqrt{t-\tau}}\right)^{\lambda-\left|\gamma^{\prime}\right|} \\
\quad \times\left(\frac{d(y)}{\left|y^{\prime}\right|}\right)^{-\varepsilon \gamma^{\prime}} \exp \left(-\frac{\kappa\left(\left|y^{\prime}\right|^{2}+\left|x^{\prime \prime}-y^{\prime \prime}\right|^{2}\right)}{t-\tau}\right)
\end{gathered}
$$

holds for $\left|\alpha^{\prime \prime}\right| \leq 2$ and $k \leq 1$. Applying the estimate

$$
\sum_{\left|\alpha^{\prime}\right| \leq 1}\left|x^{\prime}\right|^{\beta-2+\left|\alpha^{\prime}\right|+m / p}\left|\partial_{x^{\prime}}^{\alpha^{\prime}} v(x, y, t, \tau)\right| \leq c\left\|v\left(\cdot, x^{\prime \prime}, y, t, \tau\right)\right\|_{V_{p ; \beta}^{2}(K)}
$$

for $v(x, y, t, \tau)=\chi\left(x^{\prime}, t-\tau\right) \partial_{t}^{k} \partial_{x^{\prime \prime}}^{\alpha^{\prime \prime}} \partial_{\tau}^{l} \partial_{y}^{\gamma} R(x, y, t, \tau), p>m$ (cf. [18, Lemma 1.2.3]), we get

$$
\begin{aligned}
\left|\partial_{t}^{k} \partial_{\tau}^{l} \partial_{x}^{\alpha} \partial_{y}^{\gamma} R(x, y, t, \tau)\right| \leq & c(t-\tau)^{-k-l-(n+|\alpha|+|\gamma|) / 2}\left(\frac{\left|x^{\prime}\right|}{\sqrt{t-\tau}}\right)^{\mu-\left|\alpha^{\prime}\right|}\left(\frac{\left|y^{\prime}\right|}{\sqrt{t-\tau}}\right)^{\lambda-|\gamma|} \\
& \times\left(\frac{d(y)}{|y|}\right)^{-\varepsilon_{\gamma}} \exp \left(-\frac{\kappa\left(\left|y^{\prime}\right|^{2}+\left|x^{\prime \prime}-y^{\prime \prime}\right|^{2}\right)}{t-\tau}\right)
\end{aligned}
$$

for $\left|x^{\prime}\right|<\sqrt{t-\tau}, 0<t-\tau<T,\left|\alpha^{\prime}\right| \leq 1,\left|\alpha^{\prime \prime}\right|,\left|\gamma^{\prime}\right|,\left|\gamma^{\prime \prime}\right| \leq 2$. This proves (31). 
Comparing the representation (30) with the estimate (23), we conclude that

$$
\inf _{x^{\prime \prime}, t} \lambda_{1}^{+}\left(x^{\prime \prime}, t\right) \geq \lambda_{1}^{+}(0,0)-C \sqrt{\epsilon}
$$

where $C$ is the same constant as in Theorem 3.1.

\subsection{Asymptotics of the coefficients $\psi_{j}\left(x^{\prime \prime}, y, t, \tau\right)$}

Let $G_{0}\left(x^{\prime \prime}, t ; z, y, s\right)$ be the Green function of the first boundary value problem for the operator

$$
\partial_{s}-L_{0}\left(0, x^{\prime \prime}, t, \partial_{z}\right)=\frac{\partial}{\partial s}-\sum_{i, j=1}^{n} a_{i, j}\left(0, x^{\prime \prime}, t\right) \frac{\partial^{2}}{\partial z_{i} \partial z_{j}}
$$

with constant coefficients $a_{i, j}\left(0, x^{\prime \prime}, t\right)$ depending on the parameters $x^{\prime \prime}$ and $t$. This means that

$$
\begin{aligned}
& \left(\partial_{s}-L_{0}\left(0, x^{\prime \prime}, t, \partial_{z}\right)\right) G_{0}\left(x^{\prime \prime}, t ; z, y, s\right)=\delta(z-y) \delta(s) \quad \text { for } z, y \in \mathcal{D}, s \in \mathbb{R}, \\
& G_{0}\left(x^{\prime \prime}, t ; z, y, s\right)=0 \quad \text { for } z \in \partial \mathcal{D}, y \in \mathcal{D}, s \in \mathbb{R},\left.\quad G_{0}\left(x^{\prime \prime}, t ; z, y, s\right)\right|_{s<0}=0 .
\end{aligned}
$$

We write the operator $L_{0}\left(0, x^{\prime \prime}, t, \partial_{z}\right)$ in the form

$$
L_{0}\left(0, x^{\prime \prime}, t, \partial_{z}\right)=\nabla_{z^{\prime}}^{T}\left(A^{\prime}\left(x^{\prime \prime}, t\right) \nabla_{z^{\prime}}+B\left(x^{\prime \prime}, t\right) \nabla_{z^{\prime \prime}}\right)+\nabla_{z^{\prime \prime}}^{T}\left(B^{T}\left(x^{\prime \prime}, t\right) \nabla_{z^{\prime}}+A^{\prime \prime}\left(x^{\prime \prime}, t\right) \nabla_{z^{\prime \prime}}\right),
$$

where $\nabla_{z^{\prime}}$ and $\nabla_{z^{\prime \prime}}$ denote the nabla operators in the $z^{\prime}$ - and $z^{\prime \prime}$-variables, respectively. As in Section 2, let $U=U\left(x^{\prime \prime}, t\right)$ and $W=W\left(x^{\prime \prime}, t\right)$ be square and continuously differentiable (with respect to $x^{\prime \prime}$ and $t$ ) matrices such that $U A^{\prime} U^{T}=I_{m}$ and $W\left(A^{\prime \prime}-B^{T} A^{\prime-1} B\right) W^{T}=I_{n-m}$. By Theorem 2.1, the function $G_{0}$ admits the decomposition

$$
G_{0}\left(x^{\prime \prime}, t ; z, y, s\right)=\sum_{\lambda_{j}^{+}\left(x^{\prime \prime}, t\right)<\mu} \psi_{j, 0}\left(x^{\prime \prime}, t ; z^{\prime \prime}, y, s\right) u_{j}\left(x^{\prime \prime}, t ; z^{\prime}\right)+R_{0}\left(x^{\prime \prime}, t ; z, y, s\right)
$$

if $\lambda_{1}^{+}\left(x^{\prime \prime}, t\right)<\mu<\lambda_{1}^{+}\left(x^{\prime \prime}, t\right)+1$ and $\mu \neq \lambda_{j}^{+}\left(x^{\prime \prime}, t\right)$ for all $j$. Here,

$$
\psi_{j, 0}\left(x^{\prime \prime}, t ; z^{\prime \prime}, y, s\right)=\int_{K} v_{j}\left(x^{\prime \prime}, t ; x^{\prime}\right) L_{0}\left(0, x^{\prime \prime}, t, \partial_{x^{\prime}}, 0\right) G_{0}\left(x^{\prime \prime}, t ; x^{\prime}, z^{\prime \prime}, y, s\right) d x^{\prime}
$$

(cf. (19)), the functions $u_{j}\left(x^{\prime \prime}, t ; \cdot\right)$ and $v_{j}\left(x^{\prime \prime}, t ; \cdot\right)$ are defined by (24), and $R_{0}$ satisfies the estimate in Theorem 2.1. A more explicit formula for the function $\psi_{j, 0}$ is

$$
\begin{aligned}
\psi_{j, 0}\left(x^{\prime \prime}, t ; z^{\prime \prime}, y, s\right)= & \frac{2 \pi^{(m-n) / 2}(4 s)^{-\lambda_{j}^{+}\left(x^{\prime \prime}, t\right)-n / 2}}{|\operatorname{det} A|^{1 / 2} \Gamma\left(\lambda_{j}^{+}+m / 2\right)} u_{j}\left(x^{\prime \prime}, t ; y^{\prime}\right) \\
& \times \exp \left(-\frac{q\left(x^{\prime \prime}, t ; y^{\prime}, z^{\prime \prime}-y^{\prime \prime}\right)}{4 s}\right)
\end{aligned}
$$

(cf. (13)), where $A\left(x^{\prime \prime}, t\right)$ is the coefficients matrix of the operator $L_{0}\left(0, x^{\prime \prime}, t, \partial_{z}\right)$, and

$$
q\left(x^{\prime \prime}, t ; y^{\prime}, y^{\prime \prime}\right)=\left|U y^{\prime}\right|^{2}+\left|W\left(y^{\prime \prime}+B^{T} A^{\prime-1} y^{\prime}\right)\right|^{2}
$$


is a quadratic form with respect to $y^{\prime}$ and $y^{\prime \prime}$ satisfying the inequality (14). We define $\Psi_{j, 0}\left(x^{\prime \prime}, y, t, \tau\right)=\psi_{j, 0}\left(x^{\prime \prime}, t ; x^{\prime \prime}, y, t-\tau\right)$, i.e.,

$$
\begin{aligned}
\Psi_{j, 0}\left(x^{\prime \prime}, y, t, \tau\right)= & \frac{2 \pi^{(m-n) / 2}(4 t-4 \tau)^{-\lambda_{j}^{+}\left(x^{\prime \prime}, t\right)-n / 2}}{|\operatorname{det} A|^{1 / 2} \Gamma\left(\lambda_{j}^{+}+m / 2\right)} u_{j}\left(x^{\prime \prime}, t ; y^{\prime}\right) \\
& \times \exp \left(-\frac{q\left(x^{\prime \prime}, t ; y^{\prime}, x^{\prime \prime}-y^{\prime \prime}\right)}{4(t-\tau)}\right)
\end{aligned}
$$

for $x^{\prime \prime} \in \mathbb{R}^{n-m}, y \in \mathcal{D}, \tau<t$.

Theorem 3.3 The coefficients $\psi_{j}\left(x^{\prime \prime}, y, t, \tau\right)$ in Theorem 3.2 admit the decomposition

$$
\psi_{j}\left(x^{\prime \prime}, y, t, \tau\right)=\Psi_{j, 0}\left(x^{\prime \prime}, y, t, \tau\right)+r_{j}\left(x^{\prime \prime}, y, t, \tau\right),
$$

where $r_{j}$ satisfies the estimate

$$
\left|r_{j}\left(x^{\prime \prime}, y, t, \tau\right)\right| \leq c(t-\tau)^{-\left(n-1+\lambda_{j}^{+}\left(x^{\prime \prime}, t\right)\right) / 2}\left(\frac{\left|y^{\prime}\right|}{\sqrt{t-\tau}}\right)^{\lambda} \exp \left(-\frac{\kappa\left(\left|y^{\prime}\right|^{2}+\left|x^{\prime \prime}-y^{\prime \prime}\right|^{2}\right)}{t-\tau}\right)
$$

for $0<t-\tau<T, \lambda<\lambda_{1}^{+}(0)-C \sqrt{\epsilon}$.

Proof For shortness, we write $\lambda_{j}^{+}$instead of $\lambda_{j}^{+}\left(x^{\prime \prime}, t\right)$ in the proof of this theorem. Since $\left(\partial_{s}-L_{0}\left(0, x^{\prime \prime}, t, \partial_{y}\right)\right) G_{0}\left(x^{\prime \prime}, t ; x, y, s\right)=0$ for $x, y \in \mathcal{D}, s>0$, we have

$$
\left(\partial_{s}-L_{0}\left(0, x^{\prime \prime}, t, \partial_{y}\right)\right) \psi_{j, 0}\left(x^{\prime \prime}, t ; z^{\prime \prime}, y, s\right)=0
$$

for $y \in \mathcal{D}, s>0, x^{\prime \prime}, z^{\prime \prime} \in \mathbb{R}^{n-m}, t \in \mathbb{R}$. This means that the function $\Psi_{j, 0}$ satisfies the equation

$$
\left(-\partial_{\tau}-L_{0}\left(0, x^{\prime \prime}, t, \partial_{y}\right)\right) \Psi_{j, 0}\left(x^{\prime \prime}, y, t, \tau\right)=0 \quad \text { for } y \in \mathcal{D}, \tau<t .
$$

On the other hand, it follows from (26) that

$$
\left(-\partial_{\tau}-L^{*}\left(y, \tau, \partial_{y}\right)\right) \psi_{j}\left(x^{\prime \prime}, y, t, \tau\right)=0 \quad \text { for } y \in \mathcal{D}, \tau<t .
$$

Here $L^{*}$ denotes the formally adjoint differential operator to $L$. Consequently,

$$
\left(-\partial_{\tau}-L^{*}\left(y, \tau, \partial_{y}\right)\right) r_{j}\left(x^{\prime \prime}, y, t, \tau\right)=\left(L^{*}\left(y, \tau, \partial_{y}\right)-L_{0}\left(0, x^{\prime \prime}, t, \partial_{y}\right)\right) \Psi_{j, 0}\left(x^{\prime \prime}, y, t, \tau\right)
$$

for $y \in \mathcal{D}$ and $\tau<t$. Furthermore, $r_{j}\left(x^{\prime \prime}, y, t, t\right)=0$ for $x^{\prime \prime} \in \mathbb{R}^{n-m}, y \in \mathcal{D}$. This follows from the representation

$$
r_{j}\left(x^{\prime \prime}, y, t, \tau\right)=\int_{K} v_{j}\left(x^{\prime \prime}, t ; x^{\prime}\right) L_{0}\left(0, x^{\prime \prime}, t, \partial_{x^{\prime}}, 0\right)\left(G(x, y, t, \tau)-G_{0}\left(x^{\prime \prime}, t ; x, y, t-\tau\right)\right) d x^{\prime}
$$

of the function $r_{j}=\psi_{j}-\Psi_{j, 0}$ (cf. (26) and (35)) and from the equality $G(x, y, t, t)=$ $G_{0}\left(x^{\prime \prime}, t ; x, y, 0\right)=\delta(x-y)$. Thus,

$$
r_{j}\left(x^{\prime \prime}, y, t, \tau\right)=\int_{\tau}^{t} \int_{\mathcal{D}} G(z, y, s, \tau)\left(L^{*}\left(z, s, \partial_{z}\right)-L_{0}\left(0, x^{\prime \prime}, t, \partial_{z}\right)\right) \Psi_{j, 0}\left(x^{\prime \prime}, z, t, s\right) d z d s .
$$


Since $\Psi_{j, 0}$ has the form (37), we get

$$
\begin{aligned}
& \left|\left(L^{*}\left(z, s, \partial_{z}\right)-L_{0}\left(0, x^{\prime \prime}, t, \partial_{z}\right)\right) \Psi_{j, 0}\left(x^{\prime \prime}, z, t, s\right)\right| \\
& \quad \leq c\left(\sum_{|\alpha| \leq 1} \partial_{z}^{\alpha} \Psi_{j, 0}\left(x^{\prime \prime}, z, t, s\right)+\left(\left|z^{\prime}\right|+\left|z^{\prime \prime}-x^{\prime \prime}\right|+t-s\right) \sum_{|\alpha|=2} \partial_{z}^{\alpha} \Psi_{j, 0}\left(x^{\prime \prime}, z, t, s\right)\right) \\
& \quad \leq c(t-s)^{-\lambda_{j}^{+}+(1-n) / 2}\left|z^{\prime}\right|^{\lambda_{j}^{+}-2} \exp \left(-\frac{\kappa\left(\left|z^{\prime}\right|^{2}+\left|x^{\prime \prime}-z^{\prime \prime}\right|^{2}\right)}{t-s}\right)
\end{aligned}
$$

for $0<t-s<T$, where $c$ and $\kappa$ are positive constants. The last estimate together with (23) implies

$$
\begin{aligned}
\left|r_{j}\left(x^{\prime \prime}, y, t, \tau\right)\right| \leq & \int_{\tau}^{t} \int_{\mathcal{D}}(s-\tau)^{-n / 2}(t-s)^{-\lambda_{j}^{+}+(1-n) / 2}\left|z^{\prime}\right|^{\lambda_{j}^{+}-2}\left(\frac{\left|y^{\prime}\right|}{\left|y^{\prime}\right|+\sqrt{s-\tau}}\right)^{\lambda} \\
& \times\left(\frac{\left|z^{\prime}\right|}{\left|z^{\prime}\right|+\sqrt{s-\tau}}\right)^{\lambda} \exp \left(-\frac{\kappa|z-y|^{2}}{s-\tau}\right) \\
& \times \exp \left(-\frac{\kappa\left(\left|z^{\prime}\right|^{2}+\left|x^{\prime \prime}-z^{\prime \prime}\right|^{2}\right)}{t-s}\right) d z d s
\end{aligned}
$$

for $0<t-\tau<T$, where $\lambda<\lambda_{1}^{+}(0)-C \sqrt{\epsilon}$. Using the equalities

$$
\frac{\left|z^{\prime}-y^{\prime}\right|^{2}}{s-\tau}+\frac{\left|z^{\prime}\right|^{2}}{t-s}=\frac{\left|(t-\tau) z^{\prime}-(t-s) y^{\prime}\right|^{2}}{(t-\tau)(t-s)(s-\tau)}+\frac{\left|y^{\prime}\right|^{2}}{t-\tau}
$$

and

$$
\frac{\left|z^{\prime \prime}-y^{\prime \prime}\right|^{2}}{s-\tau}+\frac{\left|z^{\prime \prime}-x^{\prime \prime}\right|^{2}}{t-s}=\frac{\left|(t-\tau)\left(z^{\prime \prime}-x^{\prime \prime}\right)-(t-s)\left(y^{\prime \prime}-x^{\prime \prime}\right)\right|^{2}}{(t-\tau)(t-s)(s-\tau)}+\frac{\left|y^{\prime \prime}-x^{\prime \prime}\right|^{2}}{t-\tau},
$$

we obtain

$$
\begin{aligned}
\left|r_{j}\left(x^{\prime \prime}, y, t, \tau\right)\right| \leq & c \exp \left(-\frac{\kappa\left(\left|y^{\prime}\right|^{2}+\left|x^{\prime \prime}-y^{\prime \prime}\right|^{2}\right)}{t-\tau}\right) \int_{\tau}^{t} \int_{K}(s-\tau)^{-n / 2}(t-s)^{-\lambda_{j}^{+}+(1-n) / 2}\left|z^{\prime}\right|^{\lambda_{j}^{+}-2} \\
& \times\left(\frac{\left|y^{\prime}\right|}{\left|y^{\prime}\right|+\sqrt{s-\tau}}\right)^{\lambda}\left(\frac{\left|z^{\prime}\right|}{\left|z^{\prime}\right|+\sqrt{s-\tau}}\right)^{\lambda} \exp \left(-\kappa \frac{\left|(t-\tau) z^{\prime}-(t-s) y^{\prime}\right|^{2}}{(t-\tau)(t-s)(s-\tau)}\right) \\
& \times\left(\int_{\mathbb{R}^{n-m}} \exp \left(-\kappa \frac{\left|(t-\tau)\left(z^{\prime \prime}-x^{\prime \prime}\right)-(t-s)\left(y^{\prime \prime}-x^{\prime \prime}\right)\right|^{2}}{(t-\tau)(t-s)(s-\tau)}\right) d z^{\prime \prime}\right) d z^{\prime} d s .
\end{aligned}
$$

The inner integral over $\mathbb{R}^{n-m}$ is equal to $(t-s)^{(n-m) / 2}(s-\tau)^{(n-m) / 2}(t-\tau)^{(m-n) / 2}$. Substituting

$$
z^{\prime}=\xi^{\prime} \sqrt{t-s}, \quad y^{\prime}=\eta^{\prime} \sqrt{t-\tau}, \quad t-s=s^{\prime}(t-\tau), \quad s-\tau=\left(1-s^{\prime}\right)(t-\tau),
$$

we obtain

$$
\begin{aligned}
\left|r_{j}\left(x^{\prime \prime}, y, t, \tau\right)\right| \leq & c(t-\tau)^{-\left(\lambda_{j}^{+}+n-1\right) / 2} \exp \left(-\frac{\kappa\left(\left|y^{\prime}\right|^{2}+\left|x^{\prime \prime}-y^{\prime \prime}\right|^{2}\right)}{t-\tau}\right) \\
& \times \int_{0}^{1} \int_{K} F\left(\xi^{\prime}, \eta^{\prime}, s^{\prime}\right) d \xi^{\prime} d s^{\prime},
\end{aligned}
$$


where

$$
\begin{aligned}
F\left(\xi^{\prime}, \eta^{\prime}, s\right)= & s^{-\left(\lambda_{j}^{+}+1\right) / 2}(1-s)^{-m / 2}\left|\xi^{\prime}\right|^{\lambda_{j}^{+}-2}\left(\frac{\left|\xi^{\prime}\right| \sqrt{s}}{\left|\xi^{\prime}\right| \sqrt{s}+\sqrt{1-s}}\right)^{\lambda} \\
& \times\left(\frac{\left|\eta^{\prime}\right|}{\left|\eta^{\prime}\right|+\sqrt{1-s}}\right)^{\lambda} \exp \left(-\frac{\kappa\left|\xi^{\prime}-\eta^{\prime} \sqrt{s}\right|^{2}}{1-s}\right) .
\end{aligned}
$$

Let $K_{1}=\left\{\xi^{\prime} \in K:\left|\xi^{\prime}-\eta^{\prime} \sqrt{s}\right|<2\left|\eta^{\prime}\right| \sqrt{s}\right\}$ and $K_{2}=K \backslash K_{1}$. We may assume that $\lambda>0$. Then obviously

$$
\begin{aligned}
\int_{0}^{1 / 2} \int_{K_{1}} F\left(\xi^{\prime}, \eta^{\prime}, s\right) d \xi^{\prime} d s & \leq c\left(\frac{\left|\eta^{\prime}\right|}{\left|\eta^{\prime}\right|+1}\right)^{\lambda} \int_{0}^{1 / 2} \int_{\left|\xi^{\prime}\right|<3\left|\eta^{\prime}\right| \sqrt{s}} s^{\left(\lambda-\lambda_{j}^{+}-1\right) / 2}\left|\xi^{\prime}\right|^{\lambda+\lambda_{j}^{+}-2} d \xi^{\prime} d s \\
& \leq c\left(\frac{\left|\eta^{\prime}\right|}{\left|\eta^{\prime}\right|+1}\right)^{\lambda}\left|\eta^{\prime}\right|^{\lambda+\lambda_{j}^{+}-2+m} .
\end{aligned}
$$

If $\xi \in K_{2}$, then $\left|\xi^{\prime}-\eta^{\prime} \sqrt{s}\right|<2\left|\xi^{\prime}\right|<3\left|\xi^{\prime}-\eta^{\prime} \sqrt{s}\right|$. Therefore, the substitution $\xi^{\prime}-\eta^{\prime} \sqrt{s}=\zeta^{\prime}$ yields

$$
\begin{aligned}
& \int_{0}^{1 / 2} \int_{K_{2}} F\left(\xi^{\prime}, \eta^{\prime}, s\right) d \xi d s \\
& \quad \leq c\left(\frac{\left|\eta^{\prime}\right|}{\left|\eta^{\prime}\right|+1}\right)^{\lambda} \int_{0}^{1 / 2} \int_{\mathbb{R}^{m}} s^{\left(\lambda-\lambda_{j}^{+}-1\right) / 2}\left|\zeta^{\prime}\right|^{\lambda+\lambda_{j}^{+}-2} \exp \left(-\kappa\left|\zeta^{\prime}\right|^{2}\right) d \zeta^{\prime} d s .
\end{aligned}
$$

The number $\lambda$ can be chosen such that $\lambda_{j}^{+}\left(x^{\prime \prime}, t\right)<\lambda+1$ for all $x^{\prime \prime}, t$. Thus,

$$
\int_{0}^{1 / 2} \int_{K_{2}} F\left(\xi^{\prime}, \eta^{\prime}, s\right) d \xi^{\prime} d s \leq c\left(\frac{\left|\eta^{\prime}\right|}{\left|\eta^{\prime}\right|+1}\right)^{\lambda} .
$$

Next, we consider the integral of $F\left(\xi^{\prime}, \eta^{\prime}, s\right)$ for the interval $1 / 2 \leq s \leq 1$. Obviously,

$$
\begin{aligned}
& \int_{1 / 2}^{1} \int_{K} F\left(\xi^{\prime}, \eta^{\prime}, s\right) d \xi^{\prime} d s \\
& \quad \leq c \int_{1 / 2}^{1} \int_{K} \frac{\left|\xi^{\prime}\right|_{j}^{\lambda_{j}^{+}-2}}{(1-s)^{m / 2}}\left(\frac{\left|\eta^{\prime}\right|}{\left|\eta^{\prime}\right|+\sqrt{1-s}}\right)^{\lambda} \exp \left(-\frac{\kappa\left|\xi^{\prime}-\eta^{\prime} \sqrt{s}\right|^{2}}{1-s}\right) d \xi^{\prime} d s .
\end{aligned}
$$

We define

$$
\begin{aligned}
& K_{1}^{\prime}=\left\{\xi^{\prime} \in K: 2\left|\xi^{\prime}-\eta^{\prime} \sqrt{s}\right|<\left|\eta^{\prime}\right|\right\}, \\
& K_{2}^{\prime}=\left\{\xi^{\prime} \in K:\left|\xi^{\prime}-\eta^{\prime} \sqrt{s}\right|>2\left|\eta^{\prime}\right|\right\}, \quad K_{3}^{\prime}=K \backslash K_{1}^{\prime} \backslash K_{2}^{\prime} .
\end{aligned}
$$

If $\xi^{\prime} \in K_{1}^{\prime}$ and $1 / 2 \leq s \leq 1$, then $(\sqrt{2}-1)\left|\eta^{\prime}\right|<2\left|\xi^{\prime}\right|<3\left|\eta^{\prime}\right|$. Thus, the substitution $\xi^{\prime}-\eta^{\prime} \sqrt{s}=$ $\zeta^{\prime} \sqrt{1-s}$ yields

$$
\begin{aligned}
& \int_{1 / 2}^{1} \int_{K_{1}^{\prime}} F\left(\xi^{\prime}, \eta^{\prime}, s\right) d \xi^{\prime} d s \\
& \quad \leq c\left|\eta^{\prime}\right|^{\lambda_{j}^{+}-2} \int_{1 / 2}^{1}\left(\frac{\left|\eta^{\prime}\right|}{\left|\eta^{\prime}\right|+\sqrt{1-s}}\right)^{\lambda} \int_{2\left|\zeta^{\prime}\right| \sqrt{1-s<}<\eta^{\prime} \mid} \exp \left(-\kappa\left|\zeta^{\prime}\right|^{2}\right) d \zeta^{\prime} d s
\end{aligned}
$$


Kozlov and Rossmann Boundary Value Problems 2014, 2014:252

Page 21 of 37

http://www.boundaryvalueproblems.com/content/2014/1/252

$$
\begin{aligned}
& \leq c\left|\eta^{\prime}\right|^{\lambda_{j}^{+}-2} \int_{0}^{\infty}\left(\frac{\left|\eta^{\prime}\right|}{\left|\eta^{\prime}\right|+\sqrt{s}}\right)^{\lambda} \int_{2\left|\zeta^{\prime}\right| \sqrt{s}<\left|\eta^{\prime}\right|} \exp \left(-\kappa\left|\zeta^{\prime}\right|^{2}\right) d \zeta^{\prime} d s \\
& \leq c|\eta|^{\lambda_{j}^{+}-2}\left(\int_{0}^{\left|\eta^{\prime}\right|^{2}} \int_{\mathbb{R}^{m}} \exp \left(-\kappa\left|\zeta^{\prime}\right|^{2}\right) d \zeta^{\prime} d s+\int_{\left|\eta^{\prime}\right|^{2}}^{\infty}\left(\frac{\left|\eta^{\prime}\right|}{\sqrt{s}}\right)^{\lambda} \int_{2\left|\zeta^{\prime}\right| \sqrt{s}<\left|\eta^{\prime}\right|} d \zeta^{\prime} d s\right) \\
& =c\left|\eta^{\prime}\right|^{\lambda_{j}^{+}\left(x^{\prime \prime}, t\right)} .
\end{aligned}
$$

If $\xi^{\prime} \in K_{2}^{\prime}$ and $1 / 2 \leq s \leq 1$, then $\left|\eta^{\prime}\right|<\left|\xi^{\prime}\right|$ and $\left|\xi^{\prime}-\eta^{\prime} \sqrt{s}\right|<2\left|\xi^{\prime}\right|<3\left|\xi^{\prime}-\eta^{\prime} \sqrt{s}\right|$. Substituting $\xi^{\prime}-\eta^{\prime} \sqrt{s}=\zeta^{\prime} \sqrt{1-s}$, we get

$$
\begin{aligned}
& \int_{1 / 2}^{1} \int_{K_{2}^{\prime}} F\left(\xi^{\prime}, \eta^{\prime}, s\right) d \xi^{\prime} d s \\
& \quad \leq c \int_{1 / 2}^{1} \int_{\left|\zeta^{\prime}\right| \sqrt{1-s>2\left|\eta^{\prime}\right|}}(1-s)^{\left(\lambda_{j}^{+}-2\right) / 2}\left(\frac{\left|\eta^{\prime}\right|}{\left|\eta^{\prime}\right|+\sqrt{1-s}}\right)^{\lambda}\left|\zeta^{\prime}\right|^{\lambda_{j}^{+}-2} e^{-\kappa\left|\zeta^{\prime}\right|^{2}} d \zeta^{\prime} d s .
\end{aligned}
$$

We denote the integrand on the right-hand side of the last inequality by $H\left(\zeta^{\prime}, \eta^{\prime}, s\right)$. Obviously,

$$
H\left(\zeta^{\prime}, \eta^{\prime}, s\right) \leq c\left|\eta^{\prime}\right|^{-2}(1-s)^{\lambda_{j}^{+} / 2}\left|\zeta^{\prime}\right|^{\lambda_{j}^{+}} \exp \left(-\kappa\left|\zeta^{\prime}\right|^{2}\right)
$$

for $\sqrt{1-s}<\left|\eta^{\prime}\right|$ and $\left|\zeta^{\prime}\right| \sqrt{1-s}>2\left|\eta^{\prime}\right|$. On the other hand,

$$
H\left(\zeta^{\prime}, \eta^{\prime}, s\right) \leq c(1-s)^{\left(\lambda_{j}^{+}-2\right) / 2}\left(\frac{\left|\eta^{\prime}\right|}{\sqrt{1-s}}\right)^{\lambda}\left|\zeta^{\prime}\right|^{\lambda_{j}^{+}-2} \exp \left(-\kappa\left|\zeta^{\prime}\right|^{2}\right)
$$

for $\sqrt{1-s}>\left|\eta^{\prime}\right|$. Consequently,

$$
\int_{1 / 2}^{1} \int_{K_{2}^{\prime}} F\left(\xi^{\prime}, \eta^{\prime}, s\right) d \xi^{\prime} d s \leq c\left|\eta^{\prime}\right|^{-2} \int_{1-\left|\eta^{\prime}\right|^{2}}^{1}(1-s)^{\lambda_{j}^{+} / 2} d s=c^{\prime}\left|\eta^{\prime}\right|^{\lambda_{j}^{+}\left(x^{\prime \prime}, t\right)}
$$

for $\left|\eta^{\prime}\right|^{2}>1 / 2$. For $\left|\eta^{\prime}\right|^{2}<1 / 2$ we obtain

$$
\begin{aligned}
& \int_{1 / 2}^{1} \int_{K_{2}^{\prime}} F\left(\xi^{\prime}, \eta^{\prime}, s\right) d \xi^{\prime} d s \\
& \quad \leq c\left|\eta^{\prime}\right|^{-2} \int_{1-\left|\eta^{\prime}\right|^{2}}^{1}(1-s)^{\lambda_{j}^{+} / 2} d s+c\left|\eta^{\prime}\right|^{\lambda} \int_{1 / 2}^{1-\left|\eta^{\prime}\right|^{2}}(1-s)^{\left(\lambda_{j}^{+}-\lambda-2\right) / 2} d s \\
& \quad \leq c\left(\left|\eta^{\prime}\right|^{\lambda_{j}^{+}}+\left|\eta^{\prime}\right|^{\lambda}|\log | \eta^{\prime}||\right) .
\end{aligned}
$$

Finally, since $\left|\xi^{\prime}\right|<3\left|\eta^{\prime}\right|$ for $\xi^{\prime} \in K_{3}^{\prime}$, we get

$$
\begin{aligned}
& \int_{1 / 2}^{1} \int_{K_{3}^{\prime}} F\left(\xi^{\prime}, \eta^{\prime}, s\right) d \xi^{\prime} d s \\
& \quad \leq c \int_{0}^{1 / 2} \int_{\left|\xi^{\prime}\right|<3\left|\eta^{\prime}\right|} \frac{\left|\xi^{\prime}\right|^{\lambda_{j}^{+}-2}}{s^{m / 2}}\left(\frac{\left|\eta^{\prime}\right|}{\left|\eta^{\prime}\right|+\sqrt{s}}\right)^{\lambda} \exp \left(-\frac{\kappa\left|\eta^{\prime}\right|^{2}}{4 s}\right) d \xi^{\prime} d s \\
& \quad \leq c\left|\eta^{\prime}\right|^{\lambda+\lambda_{j}^{+}+m-2} \int_{0}^{\infty} \frac{\left(\left|\eta^{\prime}\right|+\sqrt{s}\right)^{-\lambda}}{s^{m / 2}} \exp \left(-\frac{\kappa\left|\eta^{\prime}\right|^{2}}{4 s}\right) d s=c^{\prime}\left|\eta^{\prime}\right|^{\lambda_{j}^{+}\left(x^{\prime \prime}, t\right)} .
\end{aligned}
$$


The above obtained estimates for the integrals of $F\left(\xi^{\prime}, \eta^{\prime}, s\right)$ together with (38) imply

$$
\left|r_{j}\left(x^{\prime \prime}, y, t, \tau\right)\right| \leq c(t-\tau)^{-\left(n-1+\lambda_{j}^{+}\left(x^{\prime \prime}, t\right)\right) / 2}\left|\eta^{\prime}\right|^{\lambda} \exp \left(-\frac{\kappa\left(\left|y^{\prime}\right|^{2}+\left|x^{\prime \prime}-y^{\prime \prime}\right|^{2}\right)}{t-\tau}\right),
$$

where $\lambda<\lambda_{1}^{+}(0)-C \sqrt{\epsilon}$. This proves the desired estimate.

\section{Asymptotics of solutions of the problem (1)}

Now, we consider the solution

$$
u(x, t)=\int_{0}^{t} \int_{\mathcal{D}} G(x, y, t, \tau) f(y, \tau) d y d \tau
$$

of the problem (1), where $G(x, y, t, \tau)$ denotes the Green function introduced in the last section. We assume that the coefficients of the operator $L\left(x, t, \partial_{x}\right)$ satisfy the same conditions (3), (21), and (22) as in the foregoing section and that $f \in L_{p ; \beta}\left(\mathcal{D}_{T}\right)=L_{p}\left(0, T ; V_{p ; \beta}^{0}(\mathcal{D})\right)$, where $p, \beta$ are such that $\mu=2-\beta-m / p$ satisfies the inequalities (29). Then by Theorem 3.2, the function $G$ has the representation

$$
G(x, y, t, \tau)=\sum_{\lambda_{j}^{+}<\mu} \psi_{j}\left(x^{\prime \prime}, y, t, \tau\right) u_{j}\left(x^{\prime \prime}, t ; x^{\prime}\right)+R(x, y, t, \tau)
$$

with a remainder $R(x, y, t, \tau)$ satisfying the estimate (31). Let $\zeta$ be an infinitely differentiable function on $\mathbb{R}_{+}=(0, \infty)$ which is equal to one on the interval $(0,1)$ and to zero on $(2, \infty)$. Furthermore, we define

$$
\chi_{1}\left(x^{\prime}, y^{\prime}\right)=\zeta\left(\frac{\left|x^{\prime}\right|}{\left|y^{\prime}\right|}\right), \quad \chi_{2}\left(x^{\prime}, t, \tau\right)=\zeta\left(\frac{\left|x^{\prime}\right|}{\sqrt{t-\tau}}\right)
$$

Obviously,

$$
u(x, t)=\sum_{\lambda_{j}^{+}<\mu} H_{j}(x, t) u_{j}\left(x^{\prime \prime}, t ; x^{\prime}\right)+v(x, t)
$$

where

$$
H_{j}(x, t)=\int_{0}^{t} \int_{\mathcal{D}} \chi_{1}\left(x^{\prime}, y^{\prime}\right) \chi_{2}\left(x^{\prime}, t, \tau\right) \psi_{j}\left(x^{\prime \prime}, y, t, \tau\right) f(y, \tau) d y d \tau
$$

and

$$
v(x, t)=\int_{0}^{t} \int_{\mathcal{D}}\left(G(x, y, t, \tau)-\chi_{1} \chi_{2} \sum_{\lambda_{j}^{+}<\mu} \psi_{j}\left(x^{\prime \prime}, y, t, \tau\right) u_{j}\left(x^{\prime \prime}, t ; x^{\prime}\right)\right) f(y, \tau) d y d \tau .
$$

We estimate the remainder $v$ and the coefficients $H_{j}$ in the decomposition (39).

\subsection{An estimate for a weighted $L_{p}$ Sobolev norm of the remainder}

Let $l$ be a nonnegative integer, and let $p, \beta$ be real numbers, $p>1$. Then the space $W_{p ; \beta}^{2 l, l}\left(\mathcal{D}_{T}\right)$ is defined as the set of all functions $u(x, t)$ on $\mathcal{D}_{T}=\mathcal{D} \times(0, T)$ with finite norm (4). An 
equivalent norm is

$$
\|u\|=\left(\int_{0}^{T} \int_{\mathcal{D}} \sum_{k=0}^{l}\left(\sum_{|\alpha|=2 l-2 k}\left|x^{\prime}\right|^{p \beta}\left|\partial_{t}^{k} \partial_{x}^{\alpha} u\right|^{p}+\left|x^{\prime}\right|^{p(\beta-2 l+2 k)}\left|\partial_{t}^{k} u\right|^{p}\right) d x d t\right)^{1 / p}
$$

(see, e.g., [18, Lemma 2.1.6]). In order to estimate the first order $x$-derivatives of the remainder $v$, we employ the following lemma (cf. [19, Lemma A.1]).

Lemma 4.1 Let $\mathcal{K}$ be the integral operator

$$
(\mathcal{K} f)(x, t)=\int_{0}^{t} \int_{\mathcal{D}} K(x, y, t, \tau) f(y, \tau) d y d \tau
$$

with a kernel $K(x, y, t, \tau)$ satisfying the estimate

$$
\begin{aligned}
|K| \leq & c(t-\tau)^{-(n+2-r) / 2}\left(\frac{\left|x^{\prime}\right|}{\left|x^{\prime}\right|+\sqrt{t-\tau}}\right)^{a+r}\left(\frac{\left|y^{\prime}\right|}{\left|y^{\prime}\right|+\sqrt{t-\tau}}\right)^{b} \\
& \times \frac{\left|x^{\prime}\right|^{\beta-r}}{\left|y^{\prime}\right|^{\beta}} \exp \left(\frac{-\kappa|x-y|^{2}}{t-\tau}\right)
\end{aligned}
$$

for $0<\tau<t<T$ and $x, y \in \mathcal{D}$, where $\kappa>0,0<r \leq 2, a+b>-m,-\frac{m}{p}-a<\beta<m-\frac{m}{p}+b$. Then $\mathcal{K}$ is bounded on $L_{p}\left(\mathcal{D}_{T}\right)$.

Analogously to [8, Lemma 2.3], we prove the following lemma.

Lemma 4.2 Suppose that $f \in L_{p ; \beta}\left(\mathcal{D}_{T}\right)$, where $p$ and $\beta$ are such that $\mu=2-\beta-m / p$ satisfies (29). Furthermore, let $v$ be the function (41). Then $\partial_{x}^{\alpha} v \in L_{p ; \beta-2+|\alpha|}\left(\mathcal{D}_{T}\right)$ for $|\alpha| \leq 1$ and

$$
\sum_{|\alpha| \leq 1}\left\|\partial_{\mathcal{x}}^{\alpha} v\right\|_{L_{p ; \beta-2+|\alpha|}\left(\mathcal{D}_{T}\right)} \leq c\|f\|_{L_{p ; \beta}\left(\mathcal{D}_{T}\right)}
$$

with a constant $c$ independent of $f$.

Proof Obviously,

$$
v=\sum_{j=1}^{3} \int_{0}^{t} \int_{\mathcal{D}} V_{j}(x, y, t, \tau) f(y, \tau) d y d \tau
$$

where

$$
\begin{aligned}
& V_{1}(x, y, t, \tau)=\chi_{2}\left(x^{\prime}, t, \tau\right) R(x, y, t, \tau), \\
& V_{2}(x, y, t, \tau)=\left(1-\chi_{2}\left(x^{\prime}, t, \tau\right)\right) G(x, y, t, \tau)
\end{aligned}
$$

and

$$
V_{3}(x, y, t, \tau)=\left(1-\chi_{1}\left(x^{\prime}, y^{\prime}\right)\right) \chi_{2}\left(x^{\prime}, t, \tau\right) \sum_{\lambda_{j}^{+}<\mu} \psi_{j}\left(x^{\prime \prime}, y, t, \tau\right) u_{j}\left(x^{\prime \prime}, t ; x^{\prime}\right) .
$$


Using Theorem 3.2, we obtain the estimate

$$
\begin{aligned}
\left|\partial_{x}^{\alpha} V_{1}(x, y, t, \tau)\right| \leq & c(t-\tau)^{-(n+|\alpha|) / 2}\left(\frac{\left|x^{\prime}\right|}{\left|x^{\prime}\right|+\sqrt{t-\tau}}\right)^{\mu-|\alpha|-\varepsilon} \\
& \times\left(\frac{\left|y^{\prime}\right|}{\left|y^{\prime}\right|+\sqrt{t-\tau}}\right)^{\lambda} \exp \left(-\frac{\kappa|x-y|^{2}}{t-\tau}\right)
\end{aligned}
$$

for $0<t-\tau<T$ and $|\alpha| \leq 1$, where $0<\lambda<\lambda_{1}^{+}(0)-C \sqrt{\epsilon}$ and $\varepsilon$ is a sufficiently small positive number. The same estimate holds for $\partial_{x}^{\alpha} V_{2}$ and $\partial_{x}^{\alpha} V_{3}$ by means of Theorem 3.1 and Lemma 3.1, respectively. Consequently by Lemma 4.1, the integral operators with the kernels $\left|x^{\prime}\right|^{\beta-2+|\alpha|}\left|y^{\prime}\right|^{-\beta} \partial_{x}^{\alpha} V_{j}(x, y, t, \tau)$ are bounded in $L_{p}\left(\mathcal{D}_{T}\right)$ for $|\alpha| \leq 1, j=1,2,3$. This proves the lemma.

Next, we estimate the $L_{p ; \beta}$ norm of $\left(\partial_{t}-L\right) \nu$.

Lemma 4.3 Suppose that $f \in L_{p ; \beta}\left(\mathcal{D}_{T}\right)$, where $p$ and $\beta$ are such that $\mu=2-\beta-m / p$ satisfies the condition (29). Then the function (41) satisfies the estimate

$$
\left\|\left(\partial_{t}-L\left(x, t, \partial_{x}\right)\right) v\right\|_{L_{p ; \beta}\left(\mathcal{D}_{T}\right)} \leq c\|f\|_{L_{p ; \beta}\left(\mathcal{D}_{T}\right)}
$$

with a constant $c$ independent of $f$.

Proof By the definition of $v$, we have

$$
\left(\partial_{t}-L\left(x, t, \partial_{x}\right)\right) v(x, t)=f(x, t)-\left(\partial_{t}-L\left(x, t, \partial_{x}\right)\right) \sum_{\lambda_{j}^{+}<\mu} H_{j}(x, t) u_{j}\left(x^{\prime \prime}, t ; x^{\prime}\right) .
$$

Here,

$$
\begin{aligned}
& \left(\partial_{t}-L\left(x, t, \partial_{x}\right)\right)\left(H_{j}(x, t) u_{j}\left(x^{\prime \prime}, t ; x^{\prime}\right)\right) \\
& \quad=\int_{-\infty}^{t} \int_{\mathcal{D}}\left(\partial_{t}-L\left(x, t, \partial_{x}\right)\right) \chi_{1}\left(x^{\prime}, y^{\prime}\right) \chi_{2}\left(x^{\prime}, t, \tau\right) \psi_{j}\left(x^{\prime \prime}, y, t, \tau\right) u_{j}\left(x^{\prime \prime}, t ; x^{\prime}\right) f(y, \tau) d y d \tau .
\end{aligned}
$$

By Lemma 3.1,

$$
\begin{aligned}
& \left|\partial_{t} \chi_{1}\left(x^{\prime}, y^{\prime}\right) \chi_{2}\left(x^{\prime}, t, \tau\right) \psi_{j}\left(x^{\prime \prime}, y, t, \tau\right) u_{j}\left(x^{\prime \prime}, t ; x^{\prime}\right)\right| \\
& \quad \leq c(t-\tau)^{-1-n / 2}\left(\frac{\left|x^{\prime}\right|}{\sqrt{t-\tau}}\right)^{\lambda_{j}^{+}\left(x^{\prime \prime}, t\right)}\left(\frac{\left|y^{\prime}\right|}{\sqrt{t-\tau}}\right)^{\lambda} \exp \left(-\frac{\kappa\left(\left|y^{\prime}\right|^{2}+\left|x^{\prime \prime}-y^{\prime \prime}\right|^{2}\right)}{t-\tau}\right)
\end{aligned}
$$

where $\lambda$ is an arbitrary positive number less than $\lambda_{1}^{+}(0)-C \sqrt{\epsilon}$. Using the fact that $\left|x^{\prime}\right|<$ $2 \sqrt{t-\tau}$ on the support of $\chi_{2}$, we obtain

$$
\begin{aligned}
& \left|x^{\prime}\right|^{\beta}\left|y^{\prime}\right|^{-\beta}\left|\partial_{t} \chi_{1}\left(x^{\prime}, y^{\prime}\right) \chi_{2}\left(x^{\prime}, t, \tau\right) \psi_{j}\left(x^{\prime \prime}, y, t, \tau\right) u_{j}\left(x^{\prime \prime}, t ; x^{\prime}\right)\right| \\
& \quad \leq c(t-\tau)^{-n / 2}\left(\frac{\left|x^{\prime}\right|}{\left|x^{\prime}\right|+\sqrt{t-\tau}}\right)^{\lambda+\lambda_{j}^{+}\left(x^{\prime \prime}, t\right)+2} \frac{\left|x^{\prime}\right|^{\beta-\lambda-2}}{\left|y^{\prime}\right|^{\beta-\lambda}} \exp \left(-\frac{\kappa|x-y|^{2}}{t-\tau}\right) .
\end{aligned}
$$


By (29) and (34), we have inf $\lambda_{j}^{+}>\mu-1$. Therefore, we can apply Lemma 4.1 (with $a=$ $\lambda+\inf \lambda_{j}^{+}$and $\left.b=0\right)$ and conclude that the operator with the kernel $\left|x^{\prime}\right|^{\beta}\left|y^{\prime}\right|^{-\beta} \partial_{t} \chi_{1} \chi_{2} \psi_{j} u_{j}$ is bounded in $L_{p}\left(\mathcal{D}_{T}\right)$. Furthermore, we obtain the estimate

$$
\begin{aligned}
& \left|x^{\prime}\right|^{\beta}\left|y^{\prime}\right|^{-\beta}\left|\left(L\left(x, t, \partial_{x}\right)-L_{0}\left(0, x^{\prime \prime}, t, \partial_{x^{\prime}}, 0\right)\right) \chi_{1}\left(x^{\prime}, y^{\prime}\right) \chi_{2}\left(x^{\prime}, t, \tau\right) \psi_{j}\left(x^{\prime \prime}, y, t, \tau\right) u_{j}\left(x^{\prime \prime}, t ; x^{\prime}\right)\right| \\
& \quad \leq c(t-\tau)^{-n / 2}\left(\frac{\left|x^{\prime}\right|}{\left|x^{\prime}\right|+\sqrt{t-\tau}}\right)^{\lambda+\lambda_{j}^{+}\left(x^{\prime \prime}, t\right)+1} \frac{\left|x^{\prime}\right|^{\beta-\lambda-2}}{\left|y^{\prime}\right|^{\beta-\lambda}} \exp \left(-\frac{\kappa|x-y|^{2}}{t-\tau}\right)
\end{aligned}
$$

by means of Lemma 3.1. Again Lemma 4.1 (with $a=\lambda+\inf \lambda_{j}^{+}-1$ and $b=0$ ) implies the boundedness of the integral operator with the kernel $\left|x^{\prime}\right|^{\beta}\left|y^{\prime}\right|^{-\beta}\left(L\left(x, t, \partial_{x}\right)-L_{0}\left(0, x^{\prime \prime}, t\right.\right.$, $\left.\left.\partial_{x^{\prime}}, 0\right)\right) \chi_{1} \chi_{2} \psi_{j} u_{j}$. Using the equality $L_{0}\left(0, x^{\prime \prime}, t, \partial_{x^{\prime}}, 0\right) u_{j}\left(x^{\prime \prime}, t ; x^{\prime}\right)=0$, one can show analogously that the integral operator with the kernel $\left|x^{\prime}\right|^{\beta}\left|y^{\prime}\right|^{-\beta} L_{0}\left(0, x^{\prime \prime}, t, \partial_{x^{\prime}}, 0\right) \chi_{1} \chi_{2} \psi_{j} u_{j}$ is bounded in $L_{p}\left(\mathcal{D}_{T}\right)$. Hence the mapping

$$
L_{p ; \beta}\left(\mathcal{D}_{T}\right) \ni f \rightarrow\left(\partial_{t}-L\left(x, t, \partial_{x}\right)\right)\left(H_{j}(x, t) u_{j}\left(x^{\prime \prime}, t ; x^{\prime}\right)\right) \in L_{p ; \beta}\left(\mathcal{D}_{T}\right)
$$

is bounded. This proves the lemma.

For the estimation of the second order derivatives of $v$, we need the following lemma.

Lemma 4.4 Let $u$ be a solution of the problem (1). If $u \in L_{p ; \beta-2}\left(\mathcal{D}_{T}\right), \partial_{x_{j}} u \in L_{p ; \beta-1}\left(\mathcal{D}_{T}\right)$ for $j=1, \ldots, n$ and $f \in L_{p ; \beta}\left(\mathcal{D}_{T}\right)$, then $u \in W_{p ; \beta}^{2,1}\left(\mathcal{D}_{T}\right)$ and

$$
\|u\|_{W_{p ; \beta}^{2,1}\left(\mathcal{D}_{T}\right)} \leq c\left(\|f\|_{L_{p ; \beta}\left(\mathcal{D}_{T}\right)}+\|u\|_{L_{p ; \beta-2}\left(\mathcal{D}_{T}\right)}+\sum_{j=1}^{n}\left\|\partial_{x_{j}} u\right\|_{L_{p ; \beta-1}\left(\mathcal{D}_{T}\right)}\right),
$$

where $c$ is independent of $u$.

Proof Let $\zeta_{v}$ be infinitely differentiable functions on $\mathcal{D}$ depending only on $r=\left|x^{\prime}\right|$ such that

$$
\operatorname{supp} \zeta_{\nu} \subset\left\{x: 2^{\nu-1}<r<2^{\nu+1}\right\}, \quad \sum_{\nu=-\infty}^{+\infty} \zeta_{\nu}=1, \quad\left|\partial_{x^{\prime}}^{\alpha} \zeta_{\nu}(x)\right| \leq c_{\alpha} 2^{-v|\alpha|}
$$

for all $\alpha$, where $c_{\alpha}$ is independent of $v$ and $x$. Then $\zeta_{v} u$ satisfies the equations

$$
\begin{aligned}
& \left(\partial_{t}-L\left(x, t, \partial_{x}\right)\right) \zeta_{\nu} u=f_{\nu} \quad \text { in } \mathcal{D} \times(0, T), \\
& \zeta_{\nu} u=0 \quad \text { on } \partial \mathcal{D} \times(0, T),\left.\quad \zeta_{\nu} u\right|_{t=0}=0 .
\end{aligned}
$$

where $f_{v}=\zeta_{v} f-\left[L\left(x, t, \partial_{x}\right), \zeta_{v}\right] u$. By [6, Theorem 1.1], the operator $\partial_{t}-\Delta_{x}$ of the heat equation realizes an isomorphism from the space

$$
\left\{u \in W_{p ; \gamma}^{2,1}\left(\mathcal{D}_{T}\right), u=0 \text { on } \partial \mathcal{D}_{T}, u(x, t)=0 \text { for } t=0\right\}
$$

onto $L_{p ; \gamma}\left(\mathcal{D}_{T}\right)$ for $\gamma+m / p=1+m / 2$. Using the coordinate transformation (9), we obtain the same result for the operator $\partial_{t}-L_{0}\left(0, \partial_{x}\right)$. Under the condition (3) on the coefficients 
of $L\left(x, t, \partial_{x}\right)$, the operator $L\left(x, t, \partial_{x}\right)-L_{0}\left(0, \partial_{x}\right)$ is small in the operator norm $W_{p ; \gamma}^{2,1}\left(\mathcal{D}_{T}\right) \rightarrow$ $L_{p ; \gamma}\left(\mathcal{D}_{T}\right)$. Consequently, the function $\zeta_{\nu} u$ satisfies the estimate

$$
\left\|\zeta_{v} u\right\|_{W_{p ; \gamma}^{2,1}\left(\mathcal{D}_{T}\right)} \leq c\left\|f_{v}\right\|_{L_{p ; \gamma}\left(\mathcal{D}_{T}\right)}
$$

with a constant $c$ independent of $f$ and $v$. Multiplying this inequality by $2^{v(\beta-\gamma)}$, we obtain

$$
\left\|\zeta_{\nu} u\right\|_{W_{p ; \beta}^{2,1}\left(\mathcal{D}_{T}\right)} \leq c\left\|f_{\nu}\right\|_{L_{p ; \beta}\left(\mathcal{D}_{T}\right)}
$$

with a constant $c$ independent of $u$ and $v$. Obviously,

$$
\left\|f_{v}\right\|_{L_{p ; \beta}\left(\mathcal{D}_{T}\right)} \leq\left\|\zeta_{v} f\right\|_{L_{p ; \beta}\left(\mathcal{D}_{T}\right)}+c\left(\left\|\eta_{\nu} u\right\|_{L_{p ; \beta-2}\left(\mathcal{D}_{T}\right)}+\left\|\eta_{v} \nabla u\right\|_{L_{p ; \beta-1}\left(\mathcal{D}_{T}\right)}\right),
$$

where $\eta_{v}=\zeta_{v-1}+\zeta_{v}+\zeta_{v+1}$ and $c$ is a constant independent of $f$ and $v$. Hence, (43) implies

$$
\left\|\zeta_{\nu} u\right\|_{W_{p ; \beta}^{2,1}\left(\mathcal{D}_{T}\right)}^{p} \leq c\left(\left\|\zeta_{\nu} f\right\|_{L_{p ; \beta}\left(\mathcal{D}_{T}\right)}^{p}+\left\|\eta_{\nu} u\right\|_{L_{p ; \beta-2}\left(\mathcal{D}_{T}\right)}^{p}+\left\|\eta_{\nu} \nabla u\right\|_{L_{p ; \beta-1}\left(\mathcal{D}_{T}\right)}^{p}\right)
$$

Summing up over all $v$, we get (42).

Using the last three lemmas, we can easily prove the following theorem.

Theorem 4.1 Suppose that $f \in L_{p ; \beta}\left(\mathcal{D}_{T}\right)$, where $p$ and $\beta$ are such that $\mu=2-\beta-m / p$ satisfies the condition (29). Then the solution u of the problem (1) admits the decomposition (39) with a remainder $v \in W_{p ; \beta}^{2,1}\left(\mathcal{D}_{T}\right)$. The coefficients $H_{j}(x, t)$ depend only on $\left|x^{\prime}\right|, x^{\prime \prime}, t$, and satisfy the estimates

$$
\left\|H_{j}\right\|_{L_{p ; \beta+\lambda_{j}^{+}-1}\left(\mathcal{D}_{T}\right)} \leq c\|f\|_{L_{p ; \beta}\left(\mathcal{D}_{T}\right)}
$$

and

$$
\left\|\partial_{t}^{l} \partial_{x}^{\alpha} H_{j}\right\|_{L_{p ; \beta+\lambda_{j}^{+}+2 l+|\alpha|-2}\left(\mathcal{D}_{T}\right)} \leq c\|f\|_{L_{p ; \beta}\left(\mathcal{D}_{T}\right)}
$$

for $1 \leq 2 l+|\alpha| \leq 2$. The constant $c$ in (44) and (45) is independent off.

Proof By Lemma 4.2, the solution $u$ has the representation (39), where $\partial_{x}^{\alpha} v \in L_{p ; \beta-2+|\alpha|}\left(\mathcal{D}_{T}\right)$ for $|\alpha| \leq 1$. Furthermore, by Lemma 4.3, $\left(\partial_{t}-L\left(x, t, \partial_{x}\right)\right) v \in L_{p ; \beta}\left(\mathcal{D}_{T}\right)$. Applying Lemma 4.4, we conclude that $v \in W_{p ; \beta}^{2,1}\left(\mathcal{D}_{T}\right)$ and

$$
\|v\|_{W_{p ; \beta}^{2,1}\left(\mathcal{D}_{T}\right)} \leq c\|f\|_{L_{p ; \beta}\left(\mathcal{D}_{T}\right)} .
$$

In order to prove (45), we have to show that the integral operator with the kernel

$$
K(x, y, t, \tau)=\left|x^{\prime}\right|^{\beta+\lambda_{j}^{+}+2 l+|\alpha|-2}\left|y^{\prime}\right|^{-\beta} \partial_{t}^{l} \partial_{x}^{\alpha} \chi_{1}\left(x^{\prime}, y^{\prime}\right) \chi_{2}\left(x^{\prime}, t, \tau\right) \psi_{j}\left(x^{\prime \prime}, y, t, \tau\right)
$$

is bounded in $L_{p}\left(\mathcal{D}_{T}\right)$. Using the estimates

$$
\left|\partial_{t}^{l_{1}} \partial_{x^{\prime}}^{\alpha^{\prime}} \chi_{1}\left(x^{\prime}, y^{\prime}\right) \chi_{2}\left(x^{\prime}, t, \tau\right)\right| \leq c\left|x^{\prime}\right|^{-\left|\alpha^{\prime}\right|}(t-\tau)^{-l_{1}}
$$


and

$$
\begin{aligned}
\left|\partial_{t}^{l_{2}} \partial_{x^{\prime \prime}}^{\alpha^{\prime \prime}} \psi_{j}\left(x^{\prime \prime}, y, t, \tau\right)\right| \leq & c(t-\tau)^{-l_{2}-\left(n+\left|\alpha^{\prime \prime}\right|+\lambda+\lambda_{j}^{+}\left(x^{\prime \prime}, t\right)\right) / 2}\left|y^{\prime}\right|^{\lambda} \\
& \times \exp \left(-\frac{\kappa\left(\left|y^{\prime}\right|^{2}+\left|x^{\prime \prime}-y^{\prime \prime}\right|^{2}\right)}{t-\tau}\right)
\end{aligned}
$$

(cf. Lemma 3.1), we obtain

$$
\begin{aligned}
|K(x, y, t, \tau)| \leq & c(t-\tau)^{-n / 2}\left(\frac{\left|x^{\prime}\right|}{\sqrt{t-\tau}}\right)^{2 l+\left|\alpha^{\prime \prime}\right|+\lambda+\lambda_{j}^{+}\left(x^{\prime \prime}, t\right)} \frac{\left|x^{\prime}\right|^{\beta-\lambda-2}}{\left|y^{\prime}\right|^{\beta-\lambda}} \\
& \times \exp \left(-\frac{\kappa\left(\left|y^{\prime}\right|^{2}+\left|x^{\prime \prime}-y^{\prime \prime}\right|^{2}\right)}{t-\tau}\right) .
\end{aligned}
$$

Since $\left|x^{\prime}\right| \leq 2 \sqrt{t-\tau}$ on the support of $\chi_{2}$, we can replace the term $\frac{\left|x^{\prime}\right|}{\sqrt{t-\tau}}$ by $\frac{\left|x^{\prime}\right|}{\left|x^{\prime}\right|+\sqrt{t-\tau}}$. Applying Lemma 4.1, we get the boundedness of the integral operator with the kernel $K(x, y, t, \tau)$. This proves (45). The estimate (44) holds analogously.

\subsection{On the coefficient in the asymptotics}

We consider the coefficients $H_{j}$ in (39) and their traces

$$
h_{j}\left(x^{\prime \prime}, t\right)=\int_{0}^{t} \int_{\mathcal{D}} \psi_{j}\left(x^{\prime \prime}, y, t, \tau\right) f(y, \tau) d y d \tau
$$

on $M \times(0, T)$. In the next lemma, we show that $h_{j}$ belongs to the anisotropic SobolevSlobodetskii space $W_{p}^{s, s / 2}\left(\mathbb{R}^{n-m} \times(0, T)\right)$ with the norm

$$
\begin{aligned}
& \|h\|_{W_{p}^{s, s / 2}\left(\mathbb{R}^{n-m} \times(0, T)\right)} \\
& \quad=\left(\int_{0}^{T}\|h(\cdot, t)\|_{W_{p}^{s}\left(\mathbb{R}^{n-m)}\right.}^{p} d t+\int_{\mathbb{R}^{n-m}}\left\|h\left(x^{\prime \prime}, \cdot\right)\right\|_{W_{p}^{s / 2}((0, T))}^{p} d x^{\prime \prime}\right)^{1 / p},
\end{aligned}
$$

where $s$ is a certain function on $\mathbb{R}^{n-m} \times(0, T)$ between 0 and 1 .

Lemma 4.5 Suppose that $f \in L_{p ; \beta}\left(\mathcal{D}_{T}\right)$, where $p$ and $\beta$ are such that $\mu=2-\beta-m / p$ satisfies the condition (29). Then the trace $h_{j}$ of the function (40) belongs to the space $W_{p}^{s, s / 2}\left(\mathbb{R}^{n-m} \times(0, T)\right)$, where $s\left(x^{\prime \prime}, t\right)=2-\beta-\lambda_{j}^{+}\left(x^{\prime \prime}, t\right)-m / p$, and it satisfies the estimate

$$
\begin{gathered}
\left\|h_{j}\right\|_{W_{p}^{s, s / 2}\left(\mathbb{R}^{\left.n-m_{\times} \times(0, T)\right)}\right.} \leq c\|f\|_{L_{p ; \beta}\left(\mathcal{D}_{T}\right)} \\
\text { for } \lambda_{j}^{+}<\mu \text {. Moreover, } t^{-s / 2} h_{j} \in L_{p}\left(\mathbb{R}^{n-m} \times(0, T)\right) \text { and } \\
\int_{0}^{T} \int_{\mathbb{R}^{n-m}} t^{-p s\left(x^{\prime \prime}, t\right) / 2}\left|h_{j}\left(x^{\prime \prime}, t\right)\right|^{p} d x^{\prime \prime} d t \leq c\|f\|_{L_{p ; \beta}\left(\mathcal{D}_{T}\right)}^{p}
\end{gathered}
$$

with a constant $c$ independent of $f$. 
Proof Note that $0<\inf s\left(x^{\prime \prime}, t\right) \leq \sup s\left(x^{\prime \prime}, t\right)<1$ under the assumptions of the theorem. Then the norm of $h$ in $W_{p}^{s, s / 2}\left(\mathbb{R}^{n-m} \times(0, T)\right)$ is equal to

$$
\begin{aligned}
& \|h\|_{W_{p}^{s, s / 2}\left(\mathbb{R}^{n-m} \times(0, T)\right)} \\
& =\left(\|h\|_{L_{p}\left(\mathbb{R}^{n-m} \times(0, T)\right)}^{p}+\int_{0}^{T} \int_{\mathbb{R}^{n-m}} \int_{\mathbb{R}^{n-m}} \frac{\left|h\left(x^{\prime \prime}, t\right)-h\left(y^{\prime \prime}, t\right)\right|^{p}}{\left|x^{\prime \prime}-y^{\prime \prime}\right|^{n-m+p s\left(x^{\prime \prime}, t\right)}} d x^{\prime \prime} d y^{\prime \prime} d t\right. \\
& \left.\quad+\int_{\mathbb{R}^{n-m}} \int_{0}^{T} \int_{0}^{T} \frac{\left|h\left(x^{\prime \prime}, t\right)-h\left(x^{\prime \prime}, \tau\right)\right|^{p}}{|t-\tau|^{1+p s\left(x^{\prime \prime}, t\right) / 2}} d t d \tau d x^{\prime \prime}\right)^{1 / p} .
\end{aligned}
$$

We consider $H_{j}$ as a function of the variables $r=\left|x^{\prime}\right|, x^{\prime \prime}$, and $t$. By (44) and (45),

$$
\int_{0}^{T} \int_{0}^{\infty} \int_{\mathbb{R}^{n-m}} r^{p(1-s)-1}\left(\left|H_{j}\right|^{p}+\left|\partial_{r} H_{j}\right|^{p}+\left|\partial_{x^{\prime \prime}} H_{j}\right|^{p}+r^{p}\left|\partial_{t} H_{j}\right|^{p}\right) d x^{\prime \prime} d r d t \leq c\|f\|_{L_{p ; \beta}\left(\mathcal{D}_{T}\right)}^{p} .
$$

Using the estimate

$$
\left\|h_{j}(\cdot, t)\right\|_{W_{p}^{s}\left(\mathbb{R}^{n-m)}\right.}^{p} \leq c \int_{0}^{\infty} \int_{\mathbb{R}^{n-m}} r^{p(1-s)-1}\left(\left|H_{j}\right|^{p}+\left|\partial_{r} H_{j}\right|^{p}+\left|\nabla_{x^{\prime \prime}} H_{j}\right|^{p}\right) d x^{\prime \prime} d r
$$

(see, e.g., [20, Section 2.9.2, Theorem 1]), where $c$ is independent of $t$, we get

$$
\int_{0}^{T}\left\|h_{j}(\cdot, t)\right\|_{W_{p}^{s}\left(\mathbb{R}^{n-m)}\right.}^{p} d t \leq c\|f\|_{L_{p ; \beta}\left(\mathcal{D}_{T}\right)}^{p}
$$

Obviously, $h_{j}\left(x^{\prime \prime}, t\right)$ is also the trace of the function $G_{j}\left(r, x^{\prime \prime}, t\right)=H_{j}\left(\sqrt{r}, x^{\prime \prime}, t\right)$. Thus,

$$
\begin{aligned}
& \left\|h_{j}\left(x^{\prime \prime}, \cdot\right)\right\|_{W_{p}^{s / 2}((0, T))}^{p} \\
& \quad \leq c \int_{0}^{\infty} \int_{0}^{T} \rho^{-1+p(2-s) / 2}\left(\left|G_{j}\left(\rho, x^{\prime \prime}, t\right)\right|^{p}+\left|\partial_{\rho} G_{j}\left(\rho, x^{\prime \prime}, t\right)\right|^{p}+\left|\partial_{t} G_{j}\left(\rho, x^{\prime \prime}, t\right)\right|^{p}\right) d t d \rho,
\end{aligned}
$$

where $c$ is independent of $x^{\prime \prime}$. Integrating with respect to $x^{\prime \prime}$ and substituting $\rho=r^{2}$, we obtain

$$
\begin{aligned}
& \int_{\mathbb{R}^{n-m}}\left\|h_{j}\left(x^{\prime \prime}, \cdot\right)\right\|_{W_{p}^{s / 2}((0, T))}^{p} d x^{\prime \prime} \\
& \quad \leq c \int_{\mathbb{R}^{n-m}} \int_{0}^{\infty} \int_{0}^{T} r^{p(1-s)-1}\left(\left|H_{j}\right|^{p}+\left|\partial_{r} H_{j}\right|^{p}+\left|r \partial_{t} H_{j}\right|^{p}\right) d t d r d x^{\prime \prime}
\end{aligned}
$$

This proves (47). Since $2-\beta-m / p>\sup \lambda_{1}^{+}$, there exist functions $a\left(x^{\prime \prime}, t\right)$ and $b\left(x^{\prime \prime}, t\right)$ such that

$$
a+b=-n-\lambda_{1}^{+}, \quad p \inf a>m-n-2 \quad \text { and } \quad p \inf b>p(\beta-n-2)+n+2 .
$$

Using the estimate

$$
\left|\psi_{j}\left(x^{\prime \prime}, y, t, \tau\right)\right| \leq c(t-\tau)^{-\left(n+\lambda_{j}^{+}\left(x^{\prime \prime}, t\right)\right) / 2} \exp \left(-\frac{\kappa\left(\left|y^{\prime}\right|^{2}+\left|x^{\prime \prime}-y^{\prime \prime}\right|^{2}\right)}{t-\tau}\right)
$$


(cf. Lemma 3.1) and Hölder's inequality, we get

$$
\begin{aligned}
\left|h_{j}\left(x^{\prime \prime}, t\right)\right| \leq & c\left(\int_{0}^{T} \int_{\mathcal{D}}(t-\tau)^{p a / 2} \tau^{(p-1) / 2}\left|y^{\prime}\right|^{p \beta}|f(y, \tau)|^{p} \exp \left(-\frac{\kappa\left|x^{\prime \prime}-y^{\prime \prime}\right|^{2}}{t-\tau}\right) d y d \tau\right)^{1 / p} \\
& \times\left(\int_{0}^{T} \int_{\mathcal{D}}(t-\tau)^{p^{\prime} b / 2} \tau^{-1 / 2}\left|y^{\prime}\right|^{-p^{\prime} \beta} \exp \left(-\frac{\kappa\left(\left|y^{\prime}\right|^{2}+\left|x^{\prime \prime}-y^{\prime \prime}\right|^{2}\right)}{t-\tau}\right) d y d \tau\right)^{1 / p^{\prime}},
\end{aligned}
$$

where $p^{\prime}=p /(p-1)$. We denote the second integral on the right-hand side of the least inequality by $I_{1}$. With the substitutions $y^{\prime}=z^{\prime} \sqrt{t-\tau}, y^{\prime \prime}=x^{\prime \prime}+z^{\prime \prime} \sqrt{t-\tau}$, and $\tau=t s$, one obtains the estimate

$$
\begin{aligned}
I_{1} & =t^{\left(p^{\prime} b-p^{\prime} \beta+n+1\right) / 2} \int_{0}^{1}(1-s)^{\left(p^{\prime} b-p^{\prime} \beta+n\right) / 2} s^{-1 / 2} d s \int_{\mathcal{D}}\left|z^{\prime}\right|^{-p^{\prime} \beta} \exp \left(-\kappa|z|^{2}\right) d z \\
& \leq c t^{\left(p^{\prime} b-p^{\prime} \beta+n+1\right) / 2} .
\end{aligned}
$$

Here, we used the fact that $-p^{\prime} \beta>-m$ and $p^{\prime}(b-\beta)+n>-2$. Hence,

$$
\begin{aligned}
\int_{0}^{T} & \int_{\mathbb{R}^{n-m}} t^{-p s / 2}\left|h_{j}\left(x^{\prime \prime}, t\right)\right|^{2} d x^{\prime \prime} d t \\
\leq & c \int_{0}^{T} \int_{\mathcal{D}}\left|y^{\prime}\right|^{p \beta}|f(y, \tau)|^{p} \tau^{(p-1) / 2} \\
& \quad \times\left(\int_{\tau}^{T} \int_{\mathbb{R}^{n-m}} t^{A}(t-\tau)^{p a / 2} \exp \left(-\frac{\kappa\left|x^{\prime \prime}-y^{\prime \prime}\right|^{2}}{t-\tau}\right) d x^{\prime \prime} d t\right) d y d \tau,
\end{aligned}
$$

where $A=\frac{p}{2}(b-\beta-s+n+1)-\frac{n+1}{2}$. Let $I_{2}$ denote the inner integral on the right-hand side of the last inequality. With the substitutions $x^{\prime \prime}=y^{\prime \prime}+z^{\prime \prime} \sqrt{t-\tau}$ and $t=\tau s$, we get

$$
\begin{aligned}
I_{2} & =\tau^{(1-p) / 2} \int_{\mathbb{R}^{n-m}} \exp \left(-\kappa\left|z^{\prime \prime}\right|^{2}\right)\left(\int_{1}^{T / \tau} s^{A\left(x^{\prime \prime}, \tau s\right)}(s-1)^{\left(p a\left(x^{\prime \prime}, \tau s\right)+n-m\right) / 2} d s\right) d x^{\prime \prime} \\
& \leq c \tau^{(1-p) / 2}
\end{aligned}
$$

since $A+(p a+n-m) / 2=-(p+1) / 2$ and $p \inf a>m-n-2$. This proves (48).

By (47) and (48), the function $h_{j}$ can be extended by zero to a function $\hat{h}_{j} \in W_{p}^{s, s / 2}\left(\mathbb{R}^{n-m} \times\right.$ $(-\infty, T))$. We introduce the following extension operator $\mathcal{E}$. Let $h$ be a function on $\mathbb{R}^{n-m} \times$ $(-\infty, T)$. Then

$$
\begin{aligned}
(\mathcal{E} h)\left(r, x^{\prime \prime}, t\right) & =\zeta(r) \int_{0}^{\infty} \int_{\mathbb{R}^{n-m}} K\left(y^{\prime \prime}, \tau\right) h\left(x^{\prime \prime}-r y^{\prime \prime}, t-r^{2} \tau\right) d y^{\prime \prime} d \tau \\
& =\zeta(r) r^{m-n-2} \int_{-\infty}^{t} \int_{\mathbb{R}^{n-m}} K\left(\frac{x^{\prime \prime}-y^{\prime \prime}}{r}, \frac{t-\tau}{r^{2}}\right) h\left(y^{\prime \prime}, \tau\right) d y^{\prime \prime} d \tau
\end{aligned}
$$

for $r>0, x^{\prime \prime} \in \mathbb{R}^{n-m},-\infty<t<T$. Here $\zeta$ is an infinitely differentiable cut-off function on $(0, \infty), \zeta(r)=1$ for $r<1, \zeta(r)=0$ for $r>2$, and $K$ is a function of the form

$$
K\left(y^{\prime \prime}, \tau\right)=\eta(\tau) \prod_{j=m+1}^{n} \eta\left(y_{j}\right),
$$


where $\eta \in C_{0}^{\infty}(\mathbb{R})$, supp $\eta \subset[0,1]$ and $\int \eta(\tau) d \tau=1$. The function $\mathcal{E} h$ can be considered as a function on $\mathcal{D} \times(-\infty, T)$ if $r=\left|x^{\prime}\right|$.

Lemma 4.6 Suppose that $h \in W_{p}^{s, s / 2}\left(\mathbb{R}^{n-m} \times(-\infty, T)\right)$, where $s$ is continuously differentiable and $0<\inf s\left(x^{\prime \prime}, t\right) \leq \sup s\left(x^{\prime \prime}, t\right)<1$. Then

$$
\|\mathcal{E} h\|_{L_{p ; 1-s-m / p}(\mathcal{D} \times(-\infty, T))} \leq c\|h\|_{W_{p}^{s, s / 2}\left(\mathbb{R}^{n-m} \times(-\infty, T)\right)}
$$

and

$$
\left\|\partial_{t}^{l} \partial_{x}^{\alpha} \mathcal{E} h\right\|_{L_{p ; 2 l+|\alpha|-s-m / p}(\mathcal{D} \times(-\infty, T))} \leq c\|h\|_{W_{p}^{s, s / 2}\left(\mathbb{R}^{n-m} \times(-\infty, T)\right)} \text { for } l+|\alpha| \geq 1,
$$

where $c$ is independent of $h$.

Proof Since $\sup s\left(x^{\prime \prime}, t\right)<1$, the $L_{p ; 1-s-m / p}(\mathcal{D} \times(-\infty, T))$-norm of $\mathcal{E} h$ can easily be estimated by the $L_{p}$-norm of $h$. We consider the $t$ - and $x^{\prime \prime}$-derivatives of $\mathcal{E} h$. Obviously,

$$
\begin{aligned}
& \partial_{t}^{l} \partial_{x^{\prime \prime}}^{\alpha^{\prime \prime}}(\mathcal{E} h)\left(r, x^{\prime \prime}, t\right) \\
& \quad=\zeta(r) r^{m-n-2-2 l-\left|\alpha^{\prime \prime}\right|} \int_{-\infty}^{t} \int_{\mathbb{R}^{n-m}} K^{\left(l, \alpha^{\prime \prime}\right)}\left(\frac{x^{\prime \prime}-y^{\prime \prime}}{r}, \frac{t-\tau}{r^{2}}\right) h\left(y^{\prime \prime}, \tau\right) d y^{\prime \prime} d \tau \\
& \quad=\zeta(r) r^{-2 l-\left|\alpha^{\prime \prime}\right|} \int_{0}^{1} \int_{\mathbb{R}^{n-m}} K^{\left(l, \alpha^{\prime \prime}\right)}\left(y^{\prime \prime}, \tau\right)\left(h\left(x^{\prime \prime}-r y^{\prime \prime}, t-r^{2} \tau\right)-h\left(x^{\prime \prime}, t\right)\right) d y^{\prime \prime} d \tau
\end{aligned}
$$

for $l+\left|\alpha^{\prime \prime}\right|>0$, where $K^{\left(l, \alpha^{\prime \prime}\right)}\left(\tau, y^{\prime \prime}\right)=\partial_{\tau}^{l} \partial_{y^{\prime \prime}}^{\alpha^{\prime \prime}} K(y, \tau)$. Consequently, $\partial_{t}^{l} \partial_{x^{\prime \prime}}^{\alpha^{\prime \prime}} \mathcal{E} h=A_{1}+A_{2}$, where

$$
A_{1}\left(r, x^{\prime \prime}, t\right)=\zeta(r) r^{-2 l-\left|\alpha^{\prime \prime}\right|} \int_{0}^{1} \int_{\mathbb{R}^{n-m}} K^{\left(l, \alpha^{\prime \prime}\right)}\left(y^{\prime \prime}, \tau\right)\left(h\left(x^{\prime \prime}, t-r^{2} \tau\right)-h\left(x^{\prime \prime}, t\right)\right) d y^{\prime \prime} d \tau
$$

and

$$
\begin{aligned}
A_{2}\left(r, x^{\prime \prime}, t\right)= & \zeta(r) r^{-2 l-\left|\alpha^{\prime \prime}\right|} \int_{0}^{1} \int_{\mathbb{R}^{n-m}} K^{\left(l, \alpha^{\prime \prime}\right)}\left(y^{\prime \prime}, \tau\right) \\
& \times\left(h\left(x^{\prime \prime}-r y^{\prime \prime}, t-r^{2} \tau\right)-h\left(x^{\prime \prime}, t-r^{2} \tau\right)\right) d y^{\prime \prime} d \tau .
\end{aligned}
$$

Here

$$
\begin{aligned}
& \int_{-\infty}^{T} \int_{\mathcal{D}} r^{p\left(2 l+\left|\alpha^{\prime \prime}\right|-s\right)-m}\left|A_{1}\right|^{p} d x d t \\
& \quad \leq c \int_{-\infty}^{T} \int_{\mathbb{R}^{n-m}} \int_{0}^{2} r^{p\left(2 l+\left|\alpha^{\prime \prime}\right|-s\right)-1}\left|A_{1}\left(r, x^{\prime \prime}, t\right)\right|^{p} d r d x^{\prime \prime} d t \\
& \quad \leq c \int_{-\infty}^{T} \int_{\mathbb{R}^{n-m}} \int_{0}^{1}\left(\int_{0}^{2} r^{-p s\left(x^{\prime \prime}, t\right)-1}\left|h\left(x^{\prime \prime}, t-r^{2} \tau\right)-h\left(x^{\prime \prime}, t\right)\right|^{p} d r\right) d \tau d x^{\prime \prime} d t .
\end{aligned}
$$

With the substitution $t-r^{2} \tau=s$ in the inner integral, we obtain

$$
\begin{aligned}
& \int_{-\infty}^{T} \int_{\mathcal{D}} r^{p\left(2 l+\left|\alpha^{\prime \prime}\right|-s\right)-m}\left|A_{1}\left(r, x^{\prime \prime}, t\right)\right|^{p} d x d t \\
& \quad \leq c \int_{\mathbb{R}^{n-m}} \int_{-\infty}^{T} \int_{-\infty}^{T} \frac{\left|h\left(x^{\prime \prime}, t\right)-h\left(x^{\prime \prime}, s\right)\right|^{p}}{|t-s|^{1+p s\left(x^{\prime \prime}, t\right) / 2}} d t d s d x^{\prime \prime} .
\end{aligned}
$$


Furthermore,

$$
\begin{aligned}
& \int_{-\infty}^{T} \int_{\mathcal{D}} r^{p\left(2 l+\left|\alpha^{\prime \prime}\right|-s\right)-m}\left|A_{2}\left(r, x^{\prime \prime}, t\right)\right|^{p} d x d t \\
& \quad \leq c \int_{-\infty}^{T} \int_{\mathbb{R}^{n-m}} \int_{0}^{2}\left(\int_{0}^{1} \int_{\left|y^{\prime \prime}\right| \leq \sqrt{n-m}} r^{-p s\left(x^{\prime \prime}, t\right)-1}\left|g\left(x^{\prime \prime}, y^{\prime \prime}, r, t, \tau\right)\right|^{p} d y^{\prime \prime} d \tau\right) d r d x^{\prime \prime} d t,
\end{aligned}
$$

where $g\left(x^{\prime \prime}, y^{\prime \prime}, r, t, \tau\right)=h\left(x^{\prime \prime}-r y^{\prime \prime}, t-r^{2} \tau\right)-h\left(x^{\prime \prime}, t-r^{2} \tau\right)$. The $L_{p}$-norm of the function

$$
\left(r^{-s\left(x^{\prime \prime}, t\right)-1 / p}-r^{s\left(x^{\prime \prime}, t-r^{2} \tau\right)-1 / p}\right) g\left(x^{\prime \prime}, y^{\prime \prime}, r, t, \tau\right)
$$

can easily be estimated by the $L_{p}$-norm of $h$, since $\left|1-r^{s\left(x^{\prime \prime}, t\right)-s\left(x^{\prime \prime}, t-r^{2} \tau\right)}\right| \leq c r$. Consequently,

$$
\int_{-\infty}^{T} \int_{\mathcal{D}} r^{p\left(2 l+\left|\alpha^{\prime \prime}\right|-s\right)-m}\left|A_{2}\left(r, x^{\prime \prime}, t\right)\right|^{p} d x d t \leq c\left(\|h\|_{L_{p}\left(\mathcal{R}^{n-m} \times(-\infty, T)\right)}^{p}+B\right),
$$

where

$$
\begin{aligned}
B & =\int_{-\infty}^{T} \int_{\mathbb{R}^{n-m}} \int_{0}^{2}\left(\int_{0}^{1} \int_{\left|y^{\prime \prime}\right| \leq \sqrt{n-m}} r^{-p s\left(x^{\prime \prime}, t-r^{2} \tau\right)-1}\left|g\left(x^{\prime \prime}, y^{\prime \prime}, r, t, \tau\right)\right|^{p} d y^{\prime \prime} d \tau\right) d r d x^{\prime \prime} d t \\
& \leq \int_{-\infty}^{T} \int_{\mathbb{R}^{n-m}} \int_{0}^{2} r^{-p s\left(x^{\prime \prime}, t\right)-1}\left(\int_{\left|y^{\prime \prime}\right| \leq \sqrt{n-m}}\left|h\left(x^{\prime \prime}-r y^{\prime \prime}, t\right)-h\left(x^{\prime \prime}, t\right)\right|^{p} d y^{\prime \prime}\right) d r d x^{\prime \prime} d t .
\end{aligned}
$$

Using the coordinates $\rho^{\prime \prime}=\left|y^{\prime \prime}\right|$ and $\omega^{\prime \prime}=y^{\prime \prime} /\left|y^{\prime \prime}\right|$ in the inner integral, we get

$$
\begin{aligned}
B \leq & \int_{-\infty}^{T} \int_{\mathbb{R}^{n-m}} \int_{0}^{2}\left(\int_{0}^{\sqrt{n-m}} \int_{S^{n-m-1}}\left(\rho^{\prime \prime}\right)^{n-m-1}\left|h\left(x^{\prime \prime}-r \rho^{\prime \prime} \omega^{\prime \prime}, t\right)-h\left(x^{\prime \prime}, t\right)\right|^{p} d \omega^{\prime \prime} d \rho^{\prime \prime}\right) \\
& \times \frac{d r d x^{\prime \prime} d t}{r^{p s\left(x^{\prime \prime}, t\right)+1}},
\end{aligned}
$$

where $S^{n-m-1}$ is the $(n-m-1)$-dimensional unit sphere. The substitution $r \rho^{\prime \prime}=r^{\prime}$ leads to the inequality

$$
\begin{aligned}
B & \leq \int_{-\infty}^{T} \int_{\mathbb{R}^{n-m}} \int_{0}^{2 \sqrt{n-m}} \int_{S^{n-m-1}}\left|h\left(x^{\prime \prime}-r^{\prime} \omega^{\prime \prime}, t\right)-h\left(x^{\prime \prime}, t\right)\right|^{p} d \omega^{\prime \prime} \frac{d r^{\prime} d x^{\prime \prime} d t}{\left(r^{\prime}\right)^{p s\left(x^{\prime \prime}, t\right)+1}} \\
& \leq c \int_{-\infty}^{T} \int_{\mathbb{R}^{n-m}} \int_{\left|z^{\prime \prime}\right| \leq 2 \sqrt{n-m}} \frac{\left|h\left(x^{\prime \prime}-z^{\prime \prime}, t\right)-h\left(x^{\prime \prime}, t\right)\right|^{p}}{\left|z^{\prime \prime}\right|^{n-m+p s\left(x^{\prime \prime}, t\right)} d z^{\prime \prime} d x^{\prime \prime} d t .}
\end{aligned}
$$

This proves the estimate (50) for $\alpha^{\prime}=0,\left|\alpha^{\prime \prime}\right|+l \geq 1$. Using the representation

$$
\begin{aligned}
\partial_{r}^{k} \partial_{t}^{l} \partial_{x^{\prime \prime}}^{\alpha^{\prime \prime}} \mathcal{E} h= & \partial_{r}^{k} r^{m-n-2-2 l-\left|\alpha^{\prime \prime}\right|} \int_{-\infty}^{t} \int_{\mathbb{R}^{n-m}} K^{\left(l, \alpha^{\prime \prime}\right)}\left(\frac{x^{\prime \prime}-y^{\prime \prime}}{r}, \frac{t-\tau}{r^{2}}\right) \\
& \times\left(h\left(y^{\prime \prime}, \tau\right)-h\left(x^{\prime \prime}, t\right)\right) d y^{\prime \prime} d \tau
\end{aligned}
$$

for $r<1$ and $k+l+\left|\alpha^{\prime \prime}\right| \geq 1$, we can analogously prove (50) in the case $\alpha^{\prime} \neq 0$.

Suppose that $h$ is a function on $\mathbb{R}^{n-m} \times(0, T)$. Then we define

$$
(\mathcal{E} h)\left(r, x^{\prime \prime}, t\right)=(\mathcal{E} \hat{h})\left(r, x^{\prime \prime}, t\right) \quad \text { for } r>0, x^{\prime \prime} \in \mathbb{R}^{n-m}, t \in(0, T) \text {, }
$$


where $\hat{h}$ is the extension of $h$ by zero to $\mathbb{R}^{n-m} \times(-\infty, T)$. As a consequence of Theorem 4.1 and Lemma 4.5, we obtain the following result.

Theorem 4.2 Suppose that $f \in L_{p ; \beta}\left(\mathcal{D}_{T}\right)$, where $p$ and $\beta$ are such that $\mu=2-\beta-m / p$ satisfies the condition (29). Then the solution u of the problem (1) admits the decomposition

$$
u(x, t)=\sum_{\lambda_{j}^{+}<\mu}\left(\mathcal{E} h_{j}\right)\left(r, x^{\prime \prime}, t\right) u_{j}\left(x^{\prime \prime}, t ; x^{\prime}\right)+v(x, t),
$$

where $u_{j}, h_{j}$ are given by (24) and (46), respectively, and $v \in W_{p ; \beta}^{2,1}\left(\mathcal{D}_{T}\right)$.

Proof It follows from Lemma 4.5 that the extension $\hat{h}_{j}$ of the function $h_{j}$ is an element of the space $W_{p}^{s, s / 2}\left(\mathbb{R}^{n-m} \times(-\infty, T)\right)$, where $s\left(x^{\prime \prime}, t\right)=2-\beta-\lambda_{j}^{+}\left(x^{\prime \prime}, t\right)-m / p$. Thus, by Lemma 4.6, the function $\mathcal{E} h_{j}$ satisfies the same estimates (44) and (45) as the function $H_{j}$ in Theorem 4.1. Moreover, by Hardy's inequality,

$$
\int_{0}^{T} \int_{\mathcal{D}} r^{-p s-m}\left|\left(\mathcal{E} h_{j}-H_{j}\right)\right|^{p} d x d t \leq c \int_{0}^{T} \int_{\mathcal{D}} r^{p-p s-m}\left|\partial_{r}\left(\mathcal{E} h_{j}-H_{j}\right)\right|^{p} d x d t \leq c^{\prime}\|f\|_{L_{p ; \beta}\left(\mathcal{D}_{T}\right)}^{p}
$$

since $\mathcal{E} h_{j}-H_{j}=0$ on $M \times(0, T)$. Thus, $\partial_{t}^{l} \partial_{x}^{\alpha}\left(\mathcal{E} h_{j}-H_{j}\right) \in L_{p ; \beta+\lambda_{j}^{+}+2 l+|\alpha|-2}\left(\mathcal{D}_{T}\right)$ for $2 l+|\alpha| \leq 2$. From this, we conclude that $\left(\mathcal{E} h_{j}-H_{j}\right) u_{j} \in W_{p ; \beta}^{2,1}\left(\mathcal{D}_{T}\right)$. Applying Theorem 4.1, we obtain the assertion of Theorem 4.2.

\section{Asymptotics of weak solutions of parabolic problems in a bounded domain with an edge}

Now let $\mathcal{G}$ be a bounded domain in $\mathbb{R}^{n}$ whose boundary is of the class $C^{1,1}$ outside the $(n-m)$-dimensional manifold $M$. We assume that for every point $\xi \in M$ there exist a neighborhood $\mathcal{U}_{\xi}$ and a diffeomorphism (a $C^{\infty}$-mapping) $\kappa$ such that $\kappa(\xi)$ is the origin and $\kappa\left(\mathcal{G} \cap \mathcal{U}_{\xi}\right)=\mathcal{D}_{\xi} \cap B_{1}$, where $\mathcal{D}_{\xi}=K_{\xi} \times \mathbb{R}^{n-m}, K_{\xi}$ is a cone in $\mathbb{R}^{m}$ with vertex at the origin, and $B_{1}$ is the unit ball in $\mathbb{R}^{n}$.

Furthermore, let $L\left(x, t, \partial_{x}\right)$ be the differential operator (2) with coefficients $a_{i j}$ and $a_{j}$ satisfying the conditions (21) and (22) (with $\mathcal{G}_{T}$ instead of $\mathcal{D} \times \mathbb{R}$ ). We assume that $f \in$ $L_{2}\left(\mathcal{G}_{T}\right)$ and $r^{\beta} f \in L_{p}\left(\mathcal{G}_{T}\right)$, where $r=r(x)$ denotes the distance of the point $x$ from $M$, and we consider the weak solution (see, e.g., [21, Section 7.1]) of the problem

$$
\begin{aligned}
& \frac{\partial u}{\partial t}-L\left(x, t, \partial_{x}\right) u=f \quad \text { in } \mathcal{G}_{T}, \\
& \left.u\right|_{x \in \partial \mathcal{G}}=0,\left.\quad u\right|_{t=0}=0,
\end{aligned}
$$

i.e., $u \in L_{2}\left(0, T ; \stackrel{\circ}{W}_{2}^{1}(\mathcal{G})\right)$ and $u_{t} \in L_{2}\left(0, T ; W_{2}^{-1}(\mathcal{G})\right)$. Our goal is to describe the behavior of the solution near a point $\xi \in M$. For the sake of simplicity, we assume that $\xi$ is the origin and that $\mathcal{G} \cap \mathcal{U}=\mathcal{D} \cap \mathcal{U}$ for a certain neighborhood $\mathcal{U}$ of the origin, where $\mathcal{D}=K \times \mathbb{R}^{n-m}$ is the same domain as in the foregoing sections.

Let $\epsilon$ be a sufficiently small positive number, and let $\left\{\zeta_{v}\right\}$ a be sufficiently fine partition of unity on $(\overline{\mathcal{G}} \cap \overline{\mathcal{U}}) \times[0, T]$. We can extend the coefficients $a_{i j}$ and $a_{j}$ of $L$ outside the support of $\zeta_{v}$ to $\mathcal{D} \times \mathbb{R}$ such that the conditions (21), (22), and

$$
\left|a_{i j}(x, t)-a_{i j}\left(x^{(v)}, t_{v}\right)\right| \leq \epsilon
$$


with a point $\left(x^{(v)}, t_{v}\right) \in \operatorname{supp} \zeta_{v}$ are satisfied. In the case $\operatorname{supp} \zeta_{v} \cap M \neq \emptyset$, we may assume that $x^{(v)} \in M$. We denote the differential operator with these coefficients by $L_{v}\left(x, t, \partial_{x}\right)$. Then $\zeta_{\nu} u$ satisfies the equations

$$
\partial_{t}\left(\zeta_{\nu} u\right)-L_{v}\left(\zeta_{\nu} u\right)=f_{v} \quad \text { in } \mathcal{D} \times \mathbb{R},\left.\quad u\right|_{x \in \partial \mathcal{D}}=0,
$$

where

$$
f_{v}=\zeta_{v} f+\left(\partial_{t} \zeta_{v}\right) u+\left[L_{v}, \zeta_{v}\right] u
$$

$\left[L_{v}, \zeta_{\nu}\right]=L_{v} \zeta_{\nu}-\zeta_{\nu} L_{v}$ is the commutator of $L_{v}$ and $\zeta_{\nu}$. By $G_{v}(x, y, t, \tau)$, we denote the Green function of the problem

$$
\partial_{t} u-L_{v} u=f \quad \text { in } \mathcal{D} \times \mathbb{R},\left.\quad u\right|_{x \in \partial \mathcal{D}}=0 .
$$

By Theorem 3.1, the function $G_{v}$ satisfies the estimate (23) with $\lambda<\lambda_{1}^{+}\left(x^{(v)}, t_{v}\right)-C \sqrt{\epsilon}$.

We define $V_{p ; \beta}^{l}(\mathcal{G})$ as the weighted Sobolev space with the norm

$$
\|u\|_{V_{p ; \beta}^{l}(\mathcal{G})}=\left(\int_{\mathcal{G}} \sum_{|\alpha| \leq l} r^{p(\beta-l+|\alpha|)}\left|\partial_{x}^{\alpha} u(x)\right|^{p} d x\right)^{1 / p} .
$$

An equivalent norm is (cf. [18, Lemma 2.1.6])

$$
\|u\|=\left(\int_{\mathcal{G}}\left(r^{p(\beta-l)}|u(x)|^{p}+\sum_{|\alpha|=l} r^{p \beta}\left|\partial_{x}^{\alpha} u(x)\right|^{p}\right) d x\right)^{1 / p} .
$$

Moreover, we define $W_{p, q ; \beta}^{2 l, l}\left(\mathcal{G}_{T}\right)$ as the set of all function $u=u(x, t)$ on $\mathcal{G}_{T}=\mathcal{G} \times(0, T)$ such that $\partial_{t}^{k} u \in L_{q}\left(0, T ; V_{p, \beta}^{2 l-2 k}(\mathcal{G})\right)$ for $k=0, \ldots, l$. The norm in this space is

$$
\|u\|_{W_{p, q ; \beta}^{2 l, l}\left(\mathcal{G}_{T}\right)}=\left(\int_{0}^{T}\left(\int_{\mathcal{G}_{|\alpha|+2 k \leq 2 l}} r^{p(\beta-2 l+2 k+|\alpha|)}\left|\partial_{t}^{k} \partial_{x}^{\alpha} u(x, t)\right|^{p} d x\right)^{q / p} d t\right)^{1 / q} .
$$

In the case $p=q$ we write $W_{p ; \beta}^{2 l, l}\left(\mathcal{G}_{T}\right)$ instead of $W_{p, p ; \beta}^{2 l, l}\left(\mathcal{G}_{T}\right)$. Furthermore, let $L_{p, q ; \beta}\left(\mathcal{G}_{T}\right)=$ $W_{p, q ; \beta}^{0,0}\left(\mathcal{G}_{T}\right)$ and $L_{p ; \beta}\left(\mathcal{G}_{T}\right)=L_{p, p ; \beta}\left(\mathcal{G}_{T}\right)$. Analogous notation is used for functions on the set $\mathcal{D}_{T}$. Furthermore, for arbitrary $\left(0, x^{\prime \prime}\right) \in M \cap \mathcal{U}$ and $t \in[0, T]$, we denote by $\mathfrak{A}\left(x^{\prime \prime}, t ; \lambda\right)$ the operator pencil (5) and by $\lambda_{j}^{+}\left(x^{\prime \prime}, t\right)$ its eigenvalues: $\cdots \leq \lambda_{2}^{-}<\lambda_{1}^{-}<2-m \leq 0<\lambda_{1}^{+} \leq$ $\lambda_{2}^{+}<\cdots$.

Lemma 5.1 Suppose that $\operatorname{supp} \zeta_{v} \cap M \neq \emptyset, f \in L_{2}\left(\mathcal{D}_{T}\right) \cap L_{p, q ; \beta}\left(\mathcal{D}_{T}\right)$ and

$$
2-m-\lambda_{1}^{+}\left(x^{(v)}, t_{v}\right)+C \sqrt{\epsilon}<2-\beta-\frac{m}{p}<\lambda_{1}^{+}\left(x^{(\nu)}, t_{v}\right)-C \sqrt{\epsilon} .
$$

Then the weak solution of the problem (54) satisfies the estimate

$$
\|u\|_{W_{p, q ; \beta}^{2,1}\left(\mathcal{D}_{T}\right)} \leq c\|f\|_{L_{p, q ; \beta}\left(\mathcal{D}_{T}\right)} .
$$

This lemma was proved in [6] for the heat equation. However, the proof of [6, Theorem 1.1] employs only the estimate (23) of the Green function. Therefore, the same result holds for the problem (54). 
Using the last lemma, we can estimate the $W_{p ; \beta}^{2,1}\left(\mathcal{G}_{T}\right)$-norm of the function $\zeta_{\nu} u$ if $u$ is a weak solution of the problem (51), (52).

Lemma 5.2 Let $u$ be the weak solution of the problem (51), (52), where $f \in L_{2}\left(\mathcal{G}_{T}\right) \cap$ $L_{p ; \beta}\left(\mathcal{G}_{T}\right)$. We assume that $\operatorname{supp} \zeta_{v} \cap M \neq \emptyset$ and that $p$ and $\beta$ satisfy the inequalities (55). Then $\zeta_{\nu} u \in W_{p ; \beta}^{2,1}\left(\mathcal{G}_{T}\right)$ and

$$
\left\|\zeta_{\nu} u\right\|_{W_{p ; \beta}^{2,1}\left(\mathcal{G}_{T}\right)} \leq c\|f\|_{L_{p ; \beta}^{2,1}\left(\mathcal{G}_{T}\right)} .
$$

Proof First, let $p \leq 2$. By our assumption, $\nabla u \in L_{2}\left(\mathcal{G}_{T}\right)$. Using Hölder's inequality, we conclude that $\nabla u \in L_{p ; \gamma}\left(\mathcal{G}_{T}\right)$ if $2 p \gamma>m(p-2)$. Consequently,

$$
f_{v} \in L_{p ; \gamma}\left(\mathcal{D}_{T}\right) \quad \text { if } \gamma \geq \beta, \gamma>\frac{m}{2}-\frac{m}{p} .
$$

We can choose $\gamma$ such that in addition the condition of Lemma 5.1 is satisfied for this number. Then Lemma 5.1 implies $\zeta_{\nu} u \in W_{p ; \gamma}^{2,1}\left(\mathcal{D}_{T}\right)$. Obviously, we obtain also $\eta_{\nu} u \in W_{p ; \gamma}^{2,1}\left(\mathcal{D}_{T}\right)$ if $\eta_{\nu}$ is a smooth cut-off function with sufficiently small support and $\zeta_{\nu} \eta_{\nu}=\zeta_{\nu}$. Then obviously $f_{v} \in L_{p ; \gamma^{\prime}}\left(\mathcal{D}_{T}\right)$, where $\gamma^{\prime}=\max (\beta, \gamma-1)$. It is evident that $\gamma^{\prime}$ also satisfies the condition of Lemma 5.1. Consequently, $\zeta_{\nu} u \in W_{p ; \gamma^{\prime}}^{2,1}\left(\mathcal{D}_{T}\right)$. Repeating this argument, we finally get $\zeta_{\nu} u \in W_{p ; \beta}^{2,1}\left(\mathcal{D}_{T}\right)$.

We consider the case $p>2$. By means of Hölder's inequality, it can easily be shown that

$$
L_{p ; \beta}\left(\mathcal{G}_{T}\right) \subset L_{q, p ; \gamma}\left(\mathcal{G}_{T}\right) \subset L_{q, 2 ; \gamma}\left(\mathcal{G}_{T}\right) \quad \text { if } q \leq p, \gamma>\beta+\frac{m}{p}-\frac{m}{q} .
$$

In particular, $L_{p ; \beta}\left(\mathcal{G}_{T}\right) \subset L_{2 ; \gamma}\left(\mathcal{G}_{T}\right)$ if $\gamma>\beta+\frac{m}{p}-\frac{m}{2}$. Hence $f_{v} \in L_{2 ; \gamma}\left(\mathcal{D}_{T}\right)$ for arbitrary $\gamma \geq 0$, $\gamma>\beta+\frac{m}{p}-\frac{m}{2}$. Here, $\gamma$ can be chosen such that

$$
2-m-\lambda_{1}^{+}\left(x^{(\nu)}, t_{\nu}\right)+C \sqrt{\epsilon}<2-\gamma-\frac{m}{2}<\lambda_{1}^{+}\left(x^{(\nu)}, t_{v}\right)-C \sqrt{\epsilon} .
$$

Then Lemma 5.1 implies $\zeta_{\nu} u \in W_{2 ; \gamma}^{2,1}\left(\mathcal{D}_{T}\right)$. Obviously, we obtain also $\eta_{\nu} u \in W_{2 ; \gamma}^{2,1}\left(\mathcal{D}_{T}\right)$ if $\eta_{\nu}$ is a smooth cut-off function with sufficiently small support and $\zeta_{\nu} \eta_{\nu}=\zeta_{\nu}$. In particular, $\eta_{\nu} u \in$ $L_{2}\left(0, T ; V_{2, \gamma}^{2}(\mathcal{D})\right)$ and $\partial_{t}\left(\eta_{\nu} u\right) \in L_{2}\left(0, T ; V_{2, \gamma}^{0}(\mathcal{D})\right)$. This implies $\eta_{\nu} u \in L_{\infty}\left(0, T ; V_{2, \gamma}^{1}(\mathcal{D})\right)$ and

$$
\left\|\eta_{\nu} u\right\|_{L_{\infty}\left(0, T ; V_{2, \gamma}^{1}(\mathcal{D})\right)}^{2} \leq c\left(\left\|\eta_{\nu} u\right\|_{L_{2}\left(0, T ; V_{2, \gamma}^{2}(\mathcal{D})\right)}^{2}+\left\|\partial_{t} \eta_{\nu} u\right\|_{L_{2}\left(0, T ; V_{2, \gamma}^{0}(\mathcal{D})\right)}^{2}\right) .
$$

Indeed, for the function $v=\eta_{\nu} u$ and $0<s<t$, we have

$$
\begin{aligned}
\|v(t)\|_{V_{2, \gamma}^{1}(\mathcal{D})}^{2} & =\|v(s)\|_{V_{2, \gamma}^{1}(\mathcal{D})}^{2}+\int_{s}^{t} \frac{d}{d \tau}\|v(\tau)\|_{V_{2, \gamma}^{1}(\mathcal{D})}^{2} d \tau \\
& =\|v(s)\|_{V_{2, \gamma}^{1}(\mathcal{D})}^{2}+2 \int_{s}^{t} \int_{\mathcal{D}}\left(r^{2 \gamma-2} v(\tau) v^{\prime}(\tau)+r^{2 \gamma} \nabla v(\tau) \cdot \nabla v^{\prime}(\tau)\right) d x d \tau \\
& \leq\|v(s)\|_{V_{2, \gamma}^{1}(\mathcal{D})}^{2}+2 \int_{0}^{T} \int_{\mathcal{D}}\left|v^{\prime}(t)\right|\left(r^{2 \gamma-2}|v(t)|+\left|\nabla \cdot\left(r^{2 \gamma} \nabla v(t)\right)\right|\right) d x d t \\
& \leq\|v(s)\|_{V_{2, \gamma}^{1}(\mathcal{D})}^{2}+c \int_{0}^{T}\left(\left\|v^{\prime}(t)\right\|_{V_{2, \gamma}^{0}(\mathcal{D})}^{2}+\|v(t)\|_{V_{2, \gamma}^{2}(\mathcal{D})}^{2}\right) d t .
\end{aligned}
$$


Integrating with respect to $s$, we get (57). Consequently, $\eta_{\nu} u \in L_{p}\left(0, T ; V_{2, \gamma}^{1}(\mathcal{D})\right)$. Since, moreover, $\zeta_{v} f \in L_{2, p ; \gamma}\left(\mathcal{D}_{T}\right)$, we conclude that $f_{v} \in L_{2, p ; \gamma}\left(\mathcal{D}_{T}\right)$.

Let $q$ be an arbitrary real number, $2 \leq q \leq p$. We prove by induction in $k=\left[\frac{n}{2}-\frac{n}{q}\right]$ that $f_{v} \in L_{q, p ; \gamma}\left(\mathcal{D}_{T}\right)$ with a certain $\gamma$ satisfying the condition

$$
2-m-\lambda_{1}^{+}\left(x^{(\nu)}, t_{v}\right)+C \sqrt{\epsilon}<2-\gamma-\frac{m}{q}<\lambda_{1}^{+}\left(x^{(\nu)}, t_{v}\right)-C \sqrt{\epsilon} .
$$

For $k=0$, this is already shown. Suppose that $1 \leq k \leq \frac{n}{2}-\frac{n}{q}<k+1$ and the assertion is proved for $\frac{n}{2}-\frac{n}{q}<k$. Obviously, there exists a number $q_{0} \in(2, q)$ such that $\frac{n}{2}-\frac{n}{q_{0}}<k$ and $\frac{n}{q_{0}}-\frac{n}{q}<1$. By the induction hypothesis, we get $f_{\nu} \in L_{q_{0}, p ; \gamma}\left(\mathcal{D}_{T}\right)$ with a certain $\gamma_{0}$ satisfying the condition

$$
2-m-\lambda_{1}^{+}\left(x^{(v)}, t_{v}\right)+C \sqrt{\epsilon}<2-\gamma_{0}-\frac{m}{q_{0}}<\lambda_{1}^{+}\left(x^{(v)}, t_{v}\right)-C \sqrt{\epsilon} .
$$

Then it follows from Lemma 5.1 that $\zeta_{\nu} u \in W_{q_{0}, p ; \gamma_{0}}^{2,1}\left(\mathcal{D}_{T}\right)$. Since the same is true for $\eta_{\nu} u$ if $\eta_{v}$ is a smooth cut-off function with sufficiently small support and $\zeta_{\nu} \eta_{v}=\zeta_{\nu}$, we obtain

$$
\left(\partial_{t} \zeta_{v}\right) u+\left[L_{v}, \zeta_{\nu}\right] u \in L_{p}\left(0, T ; V_{q_{0}, \gamma_{0}}^{1}(\mathcal{D})\right) \subset L_{p}\left(0, T ; V_{q, \gamma_{1}}^{0}(\mathcal{D})\right)=L_{q, p ; \gamma_{1}}\left(\mathcal{D}_{T}\right)
$$

where $\gamma_{1}=\gamma_{0}-1+\frac{n}{q_{0}}-\frac{n}{q}$ (cf. [18, Lemma 2.1.1]). Since moreover $\zeta_{\nu} f \in L_{q, p ; \gamma_{2}}\left(\mathcal{D}_{T}\right)$ for $\gamma_{2}>\beta+\frac{m}{p}-\frac{m}{q}$, we conclude that $f_{v} \in L_{q, p ; \gamma}\left(\mathcal{D}_{T}\right)$ for arbitrary $\gamma \geq \max \left(\gamma_{1}, \gamma_{2}\right)$. By (59), we have $2-m-\lambda_{1}^{+}\left(x^{(\nu)}, t_{v}\right)+C \sqrt{\epsilon}<2-\gamma_{j}-\frac{m}{q}$ for $j=1$ and $j=2$. Therefore, $\gamma$ can be chosen such that (58) is satisfied.

Thus, it is shown that $f_{v} \in L_{q, p ; \gamma}\left(\mathcal{D}_{T}\right)$ for arbitrary $q, 2 \leq q \leq p$, where $\gamma$ satisfies (58). In particular, for $q=p$, we get $f_{\nu} \in L_{p ; \gamma}\left(\mathcal{D}_{T}\right)$. Then Lemma 5.2 implies $\zeta_{\nu} u \in W_{p ; \gamma}^{2,1}\left(\mathcal{D}_{T}\right)$. Arguing as in the case $p<2$, we get $\zeta_{\nu} u \in W_{p ; \beta}^{2,1}\left(\mathcal{D}_{T}\right)$.

We denote by $M_{\mathcal{U}}$ the set of all $x^{\prime \prime} \in \mathbb{R}^{n-m}$ such that $\left(0, x^{\prime \prime}\right) \in M \cap \mathcal{U}$.

Theorem 5.1 Let $u$ be the weak solution of the problem (51), (52), where $f \in L_{2}\left(\mathcal{G}_{T}\right) \cap$ $L_{p ; \beta}\left(\mathcal{G}_{T}\right)$ and $p, \beta$ satisfy the inequalities

$$
\sup _{M_{\mathcal{U}} \times(0, T)} \lambda_{1}^{+}\left(x^{\prime \prime}, t\right)<2-\beta-m / p<\inf _{M_{\mathcal{U}} \times(0, T)} \lambda_{1}^{+}\left(x^{\prime \prime}, t\right)+1 .
$$

Moreover, we assume that $\lambda_{j}^{+}\left(x^{\prime \prime}, t\right) \neq 2-\beta-m / p$ for all $x^{\prime \prime}, t$ and $j=1,2,3, \ldots$. Then $u$ admits the decomposition

$$
u(x, t)=\sum_{\lambda_{j}^{+}<2-\beta-m / p}\left(\mathcal{E} h_{j}\right)(x, t) u_{j}\left(x^{\prime \prime}, t ; x^{\prime}\right)+v(x, t) \quad \text { for } x \in \mathcal{G} \cap \mathcal{U}, 0<t<T
$$

where $v \in W_{p ; \beta}^{2,1}\left(\mathcal{G}_{T}\right), u_{j}$ is given by $(24), h_{j} \in W_{p}^{s_{j}, s_{j} / 2}\left(M_{\mathcal{U}} \times(0, T)\right), s_{j}=2-\beta-\lambda_{j}^{+}-m / p$, and $\mathcal{E}$ is the extension operator introduced in the last subsection.

Proof Let $\left\{\zeta_{v}\right\}$ be the same partition of unity as above. Obviously, there exist numbers $\beta_{v}$ satisfying the inequalities (55) and $0<\beta_{v}-\beta<1$. Since $L_{p ; \beta}\left(\mathcal{G}_{T}\right) \subset L_{p ; \beta_{v}}\left(\mathcal{G}_{T}\right)$, we conclude from Lemma 5.2 that $\zeta_{\nu} u \in W_{p ; \beta_{v}}^{2,1}\left(\mathcal{G}_{T}\right)$. The same is obviously true for the function $\eta_{\nu} u$ 
if $\eta_{v}$ is a smooth cut-off function with sufficiently small support satisfying the equality $\zeta_{v} \eta_{v}=\zeta_{v}$. Hence

$$
f_{v}=\zeta_{v} f+\left(\partial_{t} \zeta_{\nu}\right) \eta_{\nu} u+\left[L_{v}, \zeta_{\nu}\right] \eta_{\nu} u \in L_{p ; \beta}\left(\mathcal{G}_{T}\right)
$$

Since the coefficients of $L_{v}$ satisfy the conditions (21), (22), and (53), we can apply Theorem 4.2 and obtain the decomposition

$$
\zeta_{v} u(x, t)=\sum_{\lambda_{j}^{+}<2-\beta-m / p}\left(\mathcal{E} h_{j, v}\right)\left(r, x^{\prime \prime}, t\right) u_{j}\left(x^{\prime \prime}, t ; x^{\prime}\right)+v_{v}(x, t)
$$

where $h_{j, v} \in W_{p}^{s_{j}, s_{j} / 2}\left(M_{\mathcal{U}} \times(0, T)\right)$ and $v_{v} \in W_{p ; \beta}^{2,1}\left(\mathcal{D}_{T}\right)$. Summing up over $v$, we obtain the assertion of the theorem.

\section{Competing interests}

The authors declare that they have no competing interests.

\section{Authors' contributions}

All authors contributed equally to the writing of this paper. All authors read and approved the final manuscript.

\section{Author details}

${ }^{1}$ Institute of Mathematics, Linköping University, Linköping, 58183, Sweden. ${ }^{2}$ Institute of Mathematics, University of Rostock, Rostock, 18051, Germany.

\section{Acknowledgements}

The paper partially arose during the stay of J Rossmann in Linköping in October 2012 and February 2014. The second author thanks the Department of Mathematics at the University of Linköping for the hospitality.

Received: 27 August 2014 Accepted: 21 November 2014 Published online: 11 December 2014

\section{References}

1. Grisvard, P: Edge behavior of the solution of an elliptic problem. Math. Nachr. 132, 281-299 (1987)

2. Kozlov, VA, Maz'ya, VG: On singularities of solutions of the first boundary value problem for the heat equation in domains with conical points II. Izv. Vysš. Učebn. Zaved., Mat. 3(298), 37-44 (1987) (in Russian)

3. de Coster, C, Nicaise, S: Singular behavior of the solution of the periodic Dirichlet heat equation in weighted $L_{p}$-Sobolev spaces. Adv. Differ. Equ. 16(3-4), 221-256 (2011)

4. Solonnikov, VA: Solvability of the classical initial-boundary-value problems for the heat-conduction equation in a dihedral angle. Zap. Nauč. Semin. LOMI 138, 146-180 (1984). Engl. transl. in: J. Sov. Math. 32, 526-546 (1986)

5. Solonnikov, VA: $L_{p}$-Estimates for solutions of the heat equation in a dihedral angle. Rend. Mat. Appl. (7) 21(1-4), 1-15 (2001)

6. Nazarov, Al: $L_{p}$-Estimates for a solution to the Dirichlet problem and to the Neumann problem for the heat equation in a wedge with edge of arbitrary codimension. J. Math. Sci. (N.Y.) 106(3), 2989-3014 (2001)

7. Kozlov, VA, Rossmann, J: Asymptotics of solutions of the heat equation in cones and dihedra. Math. Nachr. 285(11-12), 1422-1449 (2012)

8. Kozlov, V, Rossmann, J: Asymptotics of solutions of the heat equation in cones and dihedra under minimal assumptions on the boundary. Bound. Value Probl. 2012, 142 (2012). doi:10.1186/1687-2770-2012-142

9. Kweon, JR: Edge singular behavior for the heat equation on polyhedral cylinders in $\mathbb{R}^{3}$. Potential Anal. 38, 589-610 $(2013)$

10. Kozlov, VA: On the coefficients in the asymptotics of the solutions of parabolic initial boundary value problems in domains with a conical point. Sib. Mat. Zh. 29(2), 75-89 (1988)

11. Kozlov, VA: On the asymptotics of Green's function and Poisson's kernels for a parabolic problem in a cone I. Z. Anal. Anwend. 8(2), 131-151 (1989) (in Russian)

12. Kozlov, VA: On the asymptotics of Green's function and Poisson's kernels for a parabolic problem in a cone II. Z. Anal. Anwend. 10(1), 27-42 (1991) (in Russian)

13. Kozlov, VA, Rossmann, J: Estimates of Green's function for second order parabolic equations near edges. Mathematika (2015, to appear)

14. Maz'ya, VG, Plamenevskiĭ, BA: On the coefficients in the asymptotics of solutions of elliptic boundary value problems in domains with conical points. Math. Nachr. 76, $29-60$ (1977). Engl. transl. in: Am. Math. Soc. Transl. 123, 57-88 (1984)

15. Maz'ya, VG, PlamenevskiĬ, BA: Estimates in $L_{p}$ and Hölder classes and the Miranda-Agmon maximum principle for solutions of elliptic boundary value problems in domains with singular points on the boundary. Math. Nachr. $\mathbf{8 1}$ 25-82 (1978). Engl. transl. in: Am. Math. Soc. Transl. 123, 1-56 (1984)

16. Kozlov, VA, Maz'ya, V, Rossmann, J: Spectral Problems Associated with Corner Singularities of Solutions to Elliptic Equations. Mathematical Surveys and Monographs, vol. 85. Am. Math. Soc., Providence (2001) 
17. Maz'ya, VG, Plamenevskiĭ, BA: On the asymptotics of the fundamental solutions of elliptic boundary value problems in regions with conical points. Probl. Mat. Anal. 7, 100-145 (1979). Engl. transl. in: Sel. Math. Sov. 4(4), 363-397 (1985)

18. Maz'ya, V, Rossmann, J: Elliptic Equations in Polyhedral Domains. Mathematical Surveys and Monographs, vol. 162. Am. Math. Soc., Providence (2010)

19. Kozlov, VA, Nazarov, A: The Dirichlet problem for non-divergence parabolic equations with discontinuous in time coefficients. Math. Nachr. 282(9), 1220-1241 (2009)

20. Triebel, H: Interpolation Theory, Function Spaces, Differential Operators. Deutscher Verlag der Wissenschaften, Berlin (1978)

21. Evans, LC: Partial Differential Equations. Graduate Studies in Mathematics, vol. 19. Am. Math. Soc., Providence (1998)

doi:10.1186/s13661-014-0252-x

Cite this article as: Kozlov and Rossmann: Asymptotics of solutions of second order parabolic equations near conical points and edges. Boundary Value Problems 2014 2014:252

\section{Submit your manuscript to a SpringerOpen ${ }^{\circ}$ journal and benefit from:}

- Convenient online submission

- Rigorous peer review

- Immediate publication on acceptance

- Open access: articles freely available online

- High visibility within the field

- Retaining the copyright to your article 